\title{
SOBRE O PROBLEMA DE SEPARAÇÃO E SOBRE A TOPOLOGIA DE CERTAS APLICAÇÕES ESTÁVEIS
}

Walter dos Santos Motta Junior

Tese apresentada ao Instituto de

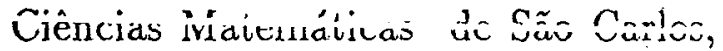
da Universidade de São Paulo, para a obtenção do Título de Doutor em Ciências (Matemática).

Orientador: Prof. Dr. Paulo Ferreira da Silva Porto Junior

São Carlos - SP

junho/1992 


\section{Meus sinceros agradecimentos,}

- ao Prof. Paulo Ferreira da Silva Porto Junior (orientador), que me introduziu no estudo de aplicações genéricas, possibilitando o contato com inúmeros matemáticos no exterior, pelo seu contínuo incentivo durante toda a realização deste trabalho;

- aos excelentes matemáticos os Profs. Carlos Biasi (ICMSC-USP), Osamu Saeki (Univ. Yamagata - Japão) e Kiyoshi Igusa (Univ. Brandeis - EUA), pelas inúmeras discussões e sugestões, as quais deram origem ao capítulo 1 deste trabalho, bem como a vários resultados dos capítulos 2 e 3 ;

- ao Prof. Luis Ernesto Mata-Lorenzo (Univ. Simon Bolivar-Venezuela), pelas discussões e sugestões referentes ao capítulo 3 ;

- ao Prof. Ozíride Manzoli Neto (ICMSC-USP), e a Profa. Maria Aparecida Soares Ruas (ICMSC-USP), pelas inúmeras discussões referentes a tópicos em topologia diferencial e algébrica;

- às Profas. Angeia Silla (IDILCE UNESP), Dirro Morhida (UFSCar) e Miriam Manoel (ICMSC-USP) e o Prof. Luiz Galante (FCT-UNESP), pelo incentivo durante a realização deste trabalho;

- aos colegas do DMT-UFMS, em especial o Prof. Alfredo Tokuda, pela concessão de afastamento para a realização deste trabalho;

- à Deus, por tudo.

Este trabalho contou com o apoio financeiro do CNPq. 


\begin{abstract}
In this work, we study the problem of separating the range of a given map, by its image; we also study the topology of certain stable maps.

By working with immersions with normal crossings, $M^{m} \rightarrow N^{m-1}$, we firstly obtain interesting results which guarantee the separation of $N$ by $f(M)$, under certain conditions.

Then, by using the Stein Factorization of a given stable map and some results on the classification of 1-connected 4-manifolds, we obtain interesting information on the topologies of the singular set and of the domain of such a map, particularly in the special generic case.

Finally, by working with stable maps whose only singularities are fold points and whose domain has low dimension, we simplify its singular set, by cancelling components by local deformations, in order to get topological information of the source.
\end{abstract}




\section{Conteúdo}

Introduçãr

1 Propriedades de Separação de Imersões com Interseções Normais em Codimensão 1

1.1 Problemas Abordados $\ldots \ldots \ldots \ldots \ldots \ldots \ldots$

1.2 Definições e Principais Resultados Relacionados . . . . . . . . . 3

1.3 Resultados Obtidos . . . . . . . . . . . . 5

2 Observações Topnlógicas Sobre Cortas Aplicacõos Genóriras Foneciais

2.1 Problemas Abordados $\ldots \ldots \ldots \ldots \ldots \ldots \ldots$

2.2 Definições e Principais Resultados Relạcionados . . . . . . . . 16

2.3 Resultados Obtidos . . . . . . . . . . . . . . . . 22

3 Sobre a Topologia de Variedades Realizando Complexos Bidimensionais

3.1 Problemas Abordados $\ldots \ldots \ldots \ldots \ldots \ldots \ldots$

3.2 Definições e Principais Resultados Relacionados . . . . . . . 36

3.3 Resultados Obtidos . . . . . . . . . . . 45

Bibliografia 


\section{Introducão}

Neste trabalho são dois os nossos objetivos: obter informações sobre o problema de separação envolvendo variedades fechadas e conexas, e, obter informações topológicas sobre o domínio e sobre o conjun to singular de certas aplicações estáveis. O trabalho está organizado da seguinte forma:

- no Capítulo 1 são abordados dojs problemas de separação, um dos quais é um questionamento sobre a recíproca do teorema de Jordan-Brouwer. Fazemos uma rápida exposição sobre os trabalhos relacionados a este tema, dos quais destacamos: Ballesteros-Fuster (1992), Biasi-Fuster (1992) e Feighn (1988). Assim, trabalhando com imersôes com interseções normais, obtemos interessantes resultados sobre o problema de separação. Dentre eles, destacamos a obtenção, sob certas condições, de uma recíproca do teorema de Jordan-Brouwer;

- no Capítulo 2 são abordados três problemas buscando a obtenção de informações topológicas sobre o domínio e sobre o conjunto singular de aplicações genéricas especiais. Uma ferramenta básica e de utilização essencial para o estudo de tais problemas é a fatorização de Stein associada a aplicação dada. Fazemos novamente uma rápida exposição dos principais trabalhos relacionados a este assunto, dos quais destacamos: Burlet-de Rham (1974), Porto-Furuya (1990), Fukuda (1987) e Saeki (1992). Com algumas restrições sobre as dimensões das variedades envolvidas, obtemos resultados que caracterizam o conjunto singular e a variedade domínjo destas aplicações. Dentre eles, destacamos uma caracterização do não-enodamento do conjunto singular na variedade domínio, onde resultados do capítulo 1 são utilizados; - no Capítulo 3 desenvolvemos um estudo sobre a simplificação do conjunto singular de certas aplicações cujas únicas singularidades apresentadas são as dobras e que tem como domínio uma variedade fechada, orientada e de baixa dimensão. É evidente a dificuldade em se formalizar procedimentos para tal simplificação. Novamente a fatorização de Stein é utilizada neste estudo. Dentre os principais trabalhos relacionados a este assunto destacam-se: Levine (1965), Mata-Lorenzo (1986) e Kobayashi (1990). Assim, trabalhando com domínios de dimensões 3 e 4, via deformações locais, podemos cancelar certas componentes singulares e assim obter informações sobre a topologia da variedade domínio das referidas aplicações com dobras. Dentre os resultados obtidos, destacamos a realização em dimensões 3 e 4 de modelos bidimensionais pré-estabelecidos. 


\section{Capítulo 1}

\section{Propriedades de Separação de Imersões com Interseções Normais em Codimensão 1}

\subsection{Problemas Abordados}

O nosso interesse, neste capítulo, é obter respostas às seguintes questões:

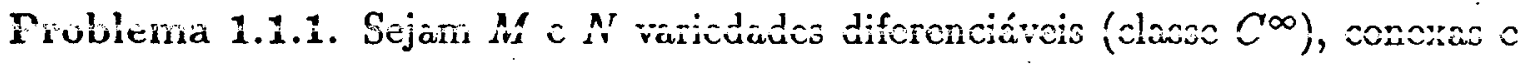
fechadas (compactas e sem bordo) de dimensões $n-1$ e $n$ respectivamente, e seja $f: M \rightarrow N$ uma imersão. Sobre quais condições é possível assegurar-se que $f(M)$ separa $N$ ?

Problema 1.1.2. Seja agora $M$ uma varidade diferenciável, conexa e fechada de dimensão $n-1$, e seja $f: M \rightarrow S^{n}$ um mergulho ( $M$ consequentemente é orientável). Então, via o teorema de separação de Jordan-Brouwer, tem-se que $f(M)$ separa a n-esfera $S^{n}$ em exatamente duas componentes conexas. Sobre quais condições é possivel assegurar-se a recíproca deste resultado? 


\subsection{Definições e Principais Resultados Relacio- nados}

Feighn (1988) considera o Problema 1.1.1., obtendo resposta afirmativa para a separação quando $f$ for uma imersão de classe $C^{2}$ e $H_{1}\left(N, Z_{2}\right)=0$. Mais especificamente, Feighn estabelece o resultado descrito abaixo, de difícil demonstração (que envolve ferramentas específicas de topologia algébrica e topologia diferenciável):

Teorema 1.2.1. (Feighn (1988)). Sejam duas variedades diferenciáveis $M$ e $N$ de dimensões $n-1$ e $n$ respectivamente, e seja $f: M \rightarrow N$ uma $C^{2}$-imersão própria. Supondo-se que $H_{1}\left(N, Z_{2}\right)=0$, então $N-f(M)$ não é conexo.

Observação 1.2.2. $O$ fato da imersão $f$ ser de classe $C^{2}$ é essencial, pois já na categoria PL, Vaccaro (1957) apresenta um contra-exemplo ao teorema acima, exibindo uma PL-imersão da esfera $S^{2}$ ("casa de duas-salas") cujo complemento em $\mathbb{R}^{3}$ é conexo.

Mais recentemente, trabalhando com aplicações 1-genéricas e quase-regulares, Ballesteros (1991), obtem um interessante resultado sobre separação, a saber:

Teorema 1.2.3. (Ballesteros (1991)). Sejam $M$ e $N$ variedades diferenciáveis de dimensōes $n-1$ e $n$ respectivamente, e seja $f: M \rightarrow N$ uma aplicação diferenciável, fechada, própria, 1-genérica e quase-regular. Então, se $H_{1}\left(N, Z_{2}\right)=0$, tem-se que $N-f(M)$ não é conexo.

Observações 1.2.4. (1) Mantendo-se as notações estabelecidas em Golubitsky-Guillemin (1973), dada uma aplicasaão diferenciável $f: X \rightarrow Y$ entre variedades diferenciáveis $X$ e $Y$, define-se as subvariedades $S_{r}$ de $J^{1}(X, Y)$ (espaço dos 1 -jatos de aplicações de $X \mathrm{em} Y) \operatorname{com} r=0,1, \ldots, \min \{\operatorname{dim} X, \operatorname{dim} Y\}$ por $S_{r}=\left\{\sigma \in J^{1}(X, Y) /\right.$ corank $\sigma=$ $r$ \}. Assim, uma aplicação diferenciável $f: X \rightarrow Y$ é dita l-genérica se $j^{1} f$ 而 $S_{r}$ 
para cada $r=0, \ldots, \min \{\operatorname{dim} X, \operatorname{dim} Y\}$. Denotando-se por $f^{(2)}$ a restrição da aplicasão $f \times f: X^{2} \rightarrow Y^{2}$ a $X^{(2)}=X^{2}-\Delta X ; f$ é dita quase-regular se $f^{(2)}$ for transversal a $\Delta Y$, onde $\Delta W$ denota a diagonal do espaço $W$.

(2) A demonstração de (1.2.3) decorre de um resultado mais geral desenvolvido por Ballesteros (1991), o qual pode ser encontrado também em Ballesteros-Fuster (1992). Neste trabalho, as condições de diferenciabilidade podem ser suprimidas e sob certas condições topológicas foi obtido também um interessante resultado envolvendo separação. Mais especificamente, Ballesteros-Fuster estabelecem que:

"Seja $f: X \rightarrow Y$ uma aplicação contínua, própria, fechada e de codimensão 1 entre variedades topológicas $X$ e $Y$, com $H_{1}\left(Y, Z_{2}\right)=0$. Se o conjunto de autointerseções de $f, A(f)=\left\{x \in X / f^{-1}(f(x)) \neq\{x\}\right\}$, for não denso em qualquer componente conexa de $X$, então $Y-f(X)$ é desconexo".

Biasi-Fuster (1992) consideram o Problema 1.1.2.. Trabalhando com imersões com interseções normais de codimensão 1 eles obtem o seguinte resultado:

Teorema 1.2.5. (Biasi-Fuster(1992)). Seja $f: M \rightarrow N$ uma aplicação diferenciável, com $M$ e $N$ variedades diferenciáveis, conexas, fechadas e de dimensões $n-1$ e $n$ respectivamente. Suponhamos que $f$ seja imersão com interseções normais, $H_{1}\left(M, Z_{2}\right)=0$ para $n>2, H_{1}\left(N, Z_{2}\right)=0$ e que o conjunto de autointerseção de $f$ seja não-vazio. Nestas condições, tem-se $\beta_{0}(N-f(M)) \geq 3$, onde $\beta_{k}(X)=\operatorname{dim} H_{k}\left(X, Z_{2}\right)$, sendo $X$ um espaço topológico.

Observações 1.2.6. (1) Seja $V$ um espaço vetorial n-dimensional e sejam $S_{1}, \ldots, S_{m}$ subespaços lineares de $V$ com codimensões dadas por $\operatorname{cod} S_{j}=n$-dimensão $S_{j}=k_{j}$. Diz-se que tais subespaços estão em "posição geral" se $\operatorname{cod}\left(\cap S_{j}\right)=\sum_{j=1}^{m} k_{j}$. Assim, uma imersão $f: M^{n-1} \rightarrow N^{n}$ é denominada "imersão com interseçôes normais" se, para cada $q \in f(M)$, as imagens pela aplicação tangente $T f: T M \rightarrow T N$, de espaços tangentes a $M$ em pontos $p_{j} \in f^{-1}(q)$, definirem um conjunto de subespaço lineares em posição geral no espaço tangente a $N$ em $q, T_{q} N$ (tais imersões são denominadas completamente regulares por Herbert (1981)). 
(2) Seja $f: M^{n-1} \rightarrow N^{n}$ una imersão com interseções normais e denotemos por $A(\subset M)$ o conjunto de auto-interseçôes de $f$ (isto é, o conjunto dos pontos múltiplos de f). Tal conjunto é estratificado por subvariedades $S_{m}: A=\cup_{m=2}^{n} S_{m}\left(\operatorname{cod} S_{m}=\right.$ $m-1$ ), onde $S_{m}$ é o conjunto de pontos de $M$ cujas $f$-imagens tem multiplicidade iii. Denota-se por $B=f(A)$, o conjunto dos valores múltiplos de $f$, o qual é também estratificado por subvariedades $B_{m}=f\left(S_{m}\right): B=\cup_{m=2}^{n} B_{m}\left(\operatorname{cod} B_{m}=\right.$ $m-2$ ). Tem-se também que $f(M), A$ e $B$ são ANR, que a restrição da aplicação $f$ a $M-A, f: M-A \rightarrow f(M)-B$, é um homeomorfismo e que as restriçôes de $f$ a $S_{m},\left.f\right|_{S_{m}}: S_{m} \rightarrow B_{m}$ são recobrimentos de m-folhas (ver Biasi-Fuster (1992)).

(3) Como consequência do teorema (1.2.5), Biasi-Fuster (1992) obtem uma recíproca do teorema de Jordan-Brouwer, a qual num certo sentido nos dá uma resposta ao Problema 1.1.2. Mais especificamente, é estabelecido que:

"Seja $f: M^{n-1} \rightarrow N^{n}$ uma imersão com interseções normais entre variedades diferenciáveis, fechadas e conexas, tal que $H_{1}\left(M, Z_{2}\right)=0$ se $n>2$ e tal que $H_{1}\left(N, Z_{2}\right)=0$. Então, $f$ é mergulho se e somente se $\beta_{0}(N-f(M))=2$ ".

(4) Uma segunda consequência de (1.2.5) estabelecida por Biasi-Fuster (1992), nos dá umá estimativa, sob certas condições, do número de componentes conexas de $N-f(M)$, a saber:

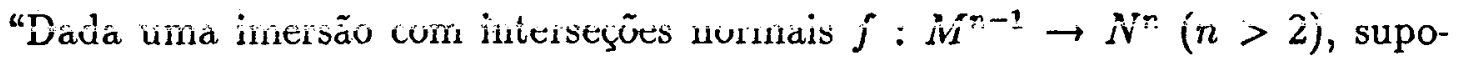
nhamos que o conjunto de auto-interseções de $f$ se reduza ao conjunto dos pontos duplos $S_{2}$. Se $H_{1}\left(M, Z_{2}\right)=H_{1}\left(N, Z_{2}\right)=0$, então $\beta_{0}(N-f(M))=2+\operatorname{dim} k e r\left(\left.f\right|_{A}\right)_{*}$, onde $\left(\left.f\right|_{A}\right)_{*}: H_{n-2}\left(A, Z_{2}\right) \rightarrow H_{n-2}\left(B, Z_{2}\right)$ é induzida por $\left(\left.f\right|_{A}\right)^{n}$.

\subsection{Resultados Obtidos}

Em tudo que segue neste capítulo, $M$ e $N$ denotam variedades diferenciáveis (classe $C^{\infty}$ ), conexas, fechadas (compactas e sem bordo) e de dimensões $n-1 \mathrm{e}$ $n$ respectivamente, $f: M \rightarrow N$ representa uma imersão com interseções normais (ver observação 1.2.6) e todas as homologias tem coeficientes em $Z_{2}$. As notações e resultados básicos utilizados abaixo seguem os textos clássicos Spanier (1966) e 
Milnor-Stasheff (1974).

Lema 1.3.1. Se $M$ for orientável e $H_{1}(N)=0$, então o fibrado normal da imersão $f, \nu(f)$, é trivial.

\section{Prova:}

Uma vez que $H_{1}(N)=0$, tem-se pelo teorema dos coeficientes universais que $H^{1}(N)=0$. Em particular, a primeira classe de Stiefel-Whitney $w_{1}(T N)$ de $T N$ é nula. Como o fibrado normal da imersão $f, \nu(f)$ é definido pela sequência exata de fibrados sobre $M$, dada por $0 \rightarrow T M \rightarrow f^{*} T N \rightarrow \nu(f) \rightarrow 0$, tem-se que $0=f^{*}\left(\omega_{1}(T N)\right)=\omega_{1}(T M)+\omega_{1}((\nu(f))$. Sendo $M$ orientada por hipótese, então $\omega_{1}(T M)=0$. Assim, tem-se que $\omega_{1}(\nu(f))=0$, o que implica que o fibrado em linha $\nu(f)$ é trivial.

O conjunto de auto-interseções (e sua estratificação via $S_{m}$ ) e o conjunto de valores múltiplos de $f$ (e sua estratificação via $B_{m}$ ) são denotados por $A$ e $B$ respectivamente, como em (1.2.6). Usaremos também a notação $A_{m}=\cup_{k \geq m} S_{k}$. Seja $[A] \in H_{n-2}(A)$ a classe fundamental módulo 2 determinada pelo lugar dos pontos múltiplos de 2 folhas $A_{2}=A$. Notemos que $[A]$ é não nula em $H_{n-2}(A)$ e que a sua existência está assegurada em Herbert (1981)(ver observações abaixo).

Observações 1.3.2. (1) - Como $f$ é imersão com intersȩ̧ões normais, en Herbert (1981), tem-se que $A$ e $B$ tem "bom comportamento", no sentido de serem subvariedades imersas admitindo classes fundamentais, mais especificamente,

(2) - Herbert (1981) estabelece que: "Cada lugar dos pontos múltiplos de m-folhas não-vazio, $A_{m}$, de uma imersão com interseções normais $f: M^{n-1} \rightarrow N^{n}$ tem em si a classe fundamental módulo 2 , unicamente determinada por sua restrição a 
subvariedade mergulhada $S_{m}=A_{m}-A_{m+1}$ ".

Lema 1.3.3. $\left(\left.f\right|_{A}\right)_{*}([A])=0$, onde $\left(\left.f\right|_{A}\right)_{*}: H_{n-2}(A) \rightarrow H_{n-2}(B)$.

Prova:

Pelas observações acima e pelo fato (ver observação 1.2.6) de $\left.f\right|_{S_{2}}: S_{2} \rightarrow f\left(S_{2}\right)$ ser um recobrimento de 2 -folhas, segue o resultado.

Lema 1.3.4. Suponhamos que $M$ seja orientável e que $H_{1}(N)=0$. Então, $j_{*}([A])=$ 0 , onde $j: A \rightarrow M$ é a aplicação inclusão.

\section{Prova:}

Utilizando-se a dualidade de Poincaré, temos apenas que demonstrar que

$$
\left(j_{*}([A])\right) \cdot \gamma=0 \text { para } \forall \gamma \in H_{1}(M), \text { onde }\left(j_{*}([A])\right) \cdot \gamma
$$

denota o número de interseção módulo 2 de $j_{*}([A])$ e $\gamma$ em $M$. Seja $c$ uma curva diferenciável (por partes) em $M$ representando $\gamma$. Sem perda de generalidade podemos assumir que $c$ não intercepta $A_{3}$ e que $c$ intercepta transversalmente $S_{2}$. Uma vez que, pelo lema 1.3.1, o fibrado normal $\nu(f)$ da imersão $f$ é trivial, nós temos uma imersão $F: M \times \mathbb{R} \rightarrow N$ tal que $\left.F\right|_{M \times\{0\}}=f$. Seja $\tilde{c}=F(c \times\{\xi\})$, onde $\xi$ é um real positivo suficientemente pequeno. Assim, temos que o número de interseção de $A_{2}$ e $c$ em $M$ é igual ao número de interseção de $f(M)$ e $\tilde{c}$ em $N$, isto é, $\left(j_{*}([A])\right) \cdot \gamma=[f(M)] .[\tilde{c}]$. Como, por hipótese, $H_{1}(N)=0$, segue-se que $[\tilde{c}]=0 \mathrm{em}$ $H_{1}(N)$. Assim, $\left(j_{*}([A])\right) \cdot \gamma=0$. Isto completa a prova.

Observação 1.3.5. Agradecemos ao Prof. Saeki pela valiosa sugestão neste lema (estabelecendo a relação de igualdade entre o número de pontos de $f(M) \cap \tilde{c}$ e 
$A \cap c)$.

Examinando a demonstração acima, podemos demonstrar um resultado mais geral, a saber:

Lema 1.3.6. Suponhamos que o fibrado normal da imersão $f, \nu(f)$, seja trivial. Se $f_{*}([M])=0$ em $H_{n-1}(N)$ ou se $f_{*}: H_{1}(M) \rightarrow H_{1}(N)$ for nula, então $j_{*}([A])=0$ em $H_{1}(M)$.

Prova:

Como $\nu(f)$ é trivial, tomando-se $F, c$ e $\tilde{c}$ como acima tem-se que $\left(j_{*}([A])\right) \cdot \gamma=$ $[f(M)] \cdot[\tilde{c}], \forall \gamma \in H_{1}(M)$. Portanto, como $[f(M)] .[\tilde{c}]=f_{*}([M]) . f_{*}([c])$ ) (por homotopia), pelas hipóteses assumidas segue o resultado.

O próximo lema tem sua demonstração baseada nos lemas 2 e 3 encontra dos em Biasi-Fuster (1992). Este lema "torna claro" os objetivos pelos quais foram estabelecidos os lemas anteriores desta seção (as notações seguem as notações dos lemas anteriores).

Abaixo apresentamos duas sequências exatas de homologia que serão fundamentais para o que pretendemos; para majores detalhes sobre a construção da sequência (b), ver Biasi-Fuster (1992):

(a) sequência de homologia do par $(N, f(M))$ :

$$
\left.0 \rightarrow H_{n}(f(M)) \rightarrow H_{n}(N) \rightarrow H_{n}(N, f(M)) \rightarrow H_{n-1}(f(M)) \stackrel{i_{*}}{\rightarrow} H_{n-1}(N)\right) \rightarrow \ldots
$$

(b) sequência de homologia construída por Biasi-Fuster (1992):

$$
\begin{gathered}
\ldots \rightarrow H_{n-1}(A) \rightarrow H_{n-1}(B) \oplus H_{n-1}(M) \rightarrow H_{n-1}(f(M)) \rightarrow H_{n-2}(A) \stackrel{\alpha}{\rightarrow} \\
\stackrel{a}{\rightarrow} H_{n-2}(B) \oplus H_{n-2}(M) \rightarrow H_{n-2}(f(M)) \rightarrow \ldots
\end{gathered}
$$




\section{Lema 1.3.7.}

(1) $\beta_{0}(N-f(M))=1+\operatorname{dim} \operatorname{ker} i_{*}$, onde $i: f(M) \rightarrow N$ é a aplicação inclusão e $i_{*}: H_{n-1}(f(M)) \rightarrow H_{n-1}(N)$.

(2) $\beta_{n-1}(f(M))=1+\operatorname{dim} \operatorname{ker} \alpha$, onde $\alpha=\left(\left.f\right|_{A}\right)_{*} \oplus j_{*}, \operatorname{com}\left(\left.f\right|_{A}\right)_{*}$ e $j_{*}$ definidas como nos lemas anteriores, $\alpha=\left(\left.f\right|_{A}\right)_{*} \oplus j_{*}: H_{n-2}(A) \rightarrow H_{n-2}(B) \oplus H_{n-2}(M)$.

\section{Prova:}

(1) Tomemos a sequência (a). Com $H_{n}(f(M))=0$ segue que $H_{n}(N, f(M))=$ $H_{n}(N) \oplus k e r i_{*}$. Logo, por dualidade, tem-se que $H_{0}(N-f(M))=H_{n}(N) \oplus$ ker $i_{*}$, o que completa a prova de (1).

(2) Tomemos a sequência (b). Como $H_{n-1}(A)=H_{n-1}(B)=0$ e $H_{n-1}(M)=Z_{2}$, da sequência (b) temos a sequência exata curta,

$$
0 \rightarrow Z_{2} \rightarrow H_{n-1}(f(M)) \rightarrow \operatorname{ker} \alpha \rightarrow 0 \text {. Logo, } H_{n-1}(f(M))=Z_{2} \oplus \text { ker } \alpha,
$$

de onde tem-se que $\beta_{\mathrm{m}=1}(f(M))=1+\operatorname{dim}$ ker $\alpha . \square$

Vamos agora então expor os resultados que obtivemos relacionados aos Problemas 1.1.1 e 1.1.2. Como resposta ao Problema 1.1.1 obtivemos o seguinte resultado,

Teorema 1.3.8. Seja $f: M \rightarrow N$ uma imersão com interseções normais entre variedades diferenciáveis $M$ e $N$, conexas, fechadas e de dimensões $n-1$ e $n$ respectivamente.

(1) Se $f_{*}([M])=0$ em $H_{n-1}(N)$, então $f(M)$ separa $N$, onde $[M] \in H_{n-1}(M)$ denota a classe fundamental de $M$; 
(2) Se dim $\operatorname{ker}\left(\left(\left.f\right|_{A}\right)_{*}: H_{n-2}(A) \rightarrow H_{n-2}(B)\right) \geq \beta_{1}(M)+\beta_{1}(N)$, então $f(M)$ separa $N$, onde $A(\subset M)$ denota o conjunto de auto-interseçôes de $f, \mathrm{e}, \beta_{i}(X)=\operatorname{dim} H_{i}(X)$, para um espaço topológico $X$;

(3) Suponhamos que $A \neq \emptyset, \beta_{1}(N) \leq 1$ e que $f_{*}: H_{1}(M) \rightarrow H_{1}(N)$ seja nula. Se o fibrado normal da imersâo $f, \nu(f)$, for trivial, então $f(M)$ separa $N$.

\section{Prova:}

(1) Suponhamos que $N-f(M)$ seja conexo e seja $x \in f(M)$ um ponto em $f(M-$ $A$ ), ou seja, $\# f^{-1}(x)=1$. Observemos que a existência de um ponto $x$ nestas condições está assegurada pelo fato de $f$ ser imersão com interseções normais. Seja $\phi:[-1,1] \rightarrow N$ um mergulho transversal a $f, \phi \pi f$, tal que $\phi([-1,1]) \cap f(M)=$ $\{x\}$. Uma vez que $N-f(M)$ é conexo, nós temos um mergulho $\tilde{\phi}: S^{1} \rightarrow N$ o qual estende $\phi$ e é tal que $\tilde{\phi}\left(S^{1}\right) \cap f(M)=\{x\}$. Como $x \in f(M-A)$, tem-se que o número de interseção módulo 2 de $f(M)$ e $\tilde{\phi}\left(S^{1}\right)$ é não nulo, e portanto o número de interseção módulo 2 de $f_{*}([M])$ e $\tilde{\phi}\left(S^{1}\right)$ é também não nulo: $\left(f_{*}([M])\right) \cdot\left[\tilde{\phi}\left(S^{1}\right)\right] \neq 0$. Por outro lado, como por hipótese temos que $f_{*}([M])=0$, então $\left(f_{*}([M])\right) \cdot\left[\tilde{\phi}\left(S^{1}\right)\right]=$ 0 (Contradição). Portanto, $N-f(M)$ é desconexo, ou seja, $f(M)$ separa $N . \square$

(2) Pelo Lema 1.3.7 (1), temos que

$$
\begin{aligned}
\beta_{0}(N-f(M))=1+\operatorname{dim} \operatorname{ker} i_{*} & \geq 1+\beta_{n-1}(f(M))-\beta_{n-1}(N)= \\
& =1+\beta_{n-1}(f(M))-\beta_{1}(N) .
\end{aligned}
$$

Por outro lado, pelo lema 1.3.7 (2), temos que

$$
\begin{aligned}
\beta_{n-1}(f(M))=1+\operatorname{dim} \operatorname{ker} \alpha & \geq 1+\operatorname{dim} \operatorname{ker}\left(\left.f\right|_{A}\right)_{*}-\beta_{n-2}(M)= \\
& =1+\operatorname{dim} \operatorname{ker}\left(\left.f\right|_{A}\right)_{*}-\beta_{1}(M) .
\end{aligned}
$$

Portanto, pode-se concluir que $\beta_{0}(N-f(M)) \geq 2+\operatorname{dim} \operatorname{ker}\left(\left.f\right|_{A}\right)_{*}-\beta_{1}(M)-$ $\beta_{1}(N)$. Assim, pela hipótese dada, tem-se então que $\beta_{0}(N-f(M)) \geq 2$. Isto completa a prova.

(3) Como por hipótese $\beta_{1}(N) \leq 1$, nós temos que $\beta_{n-1}(N)=\beta_{1}(N) \leq 1$. Logo, pelo lema 1.3.7(1) temos que $\beta_{0}(N-f(M)) \geq \beta_{n-1}(f(M))$. Pelo lema 1.3.7(2), segue que $\beta_{0}(N-f(M)) \geq 1+\operatorname{dim} \operatorname{ker} \alpha$, onde $\alpha=\left(\left.f\right|_{A}\right)_{*} \oplus j_{*}$. Assim, é suficiente demonstrarmos que existe um elemento não nulo em $H_{n-2}(A)$ tal que sua $\alpha$-imagem 
é nula em $H_{n-2}(B) \oplus H_{n-2}(M)$. Logo, pelos lemas 1.3.3 e 1.3.6, tem-se a existência deste elemento em $H_{n-2}(A)$. Isto completa a prova.

O objetivo agora é apresentar uma resposta ao Problema 1.1.2, a qual consequentemente corresponde a uma recíproca a teorema de Jordan-Brouwer. Neste sentido, é obtido o seguinte resultado:

Teorema 13.9. Seja $f: M \rightarrow N$ uma imersão com interseções normais entre variedades diferenciáveis, conexas, fechadas e de dimensões $n-1$ e $n$ respectivamente, e, suponhamos que $A(\subset M)$, o conjunto de auto-interseções de $f$, seja não nulo.

(1) Se $M$ for orientável e $H_{1}(N)=0$, então $\beta_{0}(N-f(M)) \geq 3$

(2) Se $i_{*}: H_{n-1}(f(M)) \rightarrow H_{n-1}(N)$ for nulo e se o fibrado normal da imersão $f, \nu(f)$, for trivial, então $\beta_{0}(N-f(M)) \geq 3$, onde $i: f(M) \rightarrow N$ é a aplicação inclusão.

\section{Prova:}

(1) Uma vez que $H_{1}(N)=0$, segue-se que $H_{n-1}(N)=0$, e, então pelo lema 1.3.7(1) tem-se que $\beta_{0}(N-f(M))=1+\beta_{n-1}(f(M))$. Por outro lado, pelos lemas $1.3 .3 \mathrm{e}$ 1.3.4, existe um elemento $[A] \in H_{n-2}(A)$ não nulo com $\alpha([A])=0$. Assim, pelo lèma 1.3.7(2) tem-se que $\beta_{n-1}(f(M)) \geq 2$. Portanto, $\beta_{0}(N-f(M)) \geq 3$.

(2) Como $i_{*}: H_{n-1}(f(M)) \rightarrow H_{n-1}(N)$ é nulo, pelo lema 1.3.7(1) tem-se que $\beta_{0}(N-f(M))=1+\beta_{n-1}(f(M))$. Por outro lado, pelos lemas 1.3.3 e 1.3.6, existe um elemento $[A] \in H_{n-2}(A)$ não nulo com $\alpha([A])=0$. Observemos que $f_{*}([M])=0$ em $H_{n-1}(N)$, pois $f_{*}([M])=i_{*}([f(M)])=0$. Logo, pelo lema 1.3.7(2), concluimos que $\beta_{n-1}(f(M)) \geq 2$. Portanto, $\beta_{0}(N-f(M)) \geq 3$. 
Observações 1.3.10 (1) Consideremos uma imersão com interseções normais $f$ do círculo no toro, $f: S^{1} \rightarrow T^{2}$, dada pela Figura 1.1 abaixo. Tem-se que o fibrado normal $\nu(f)$ é trivial, $f_{*}([M])=0$ e que $\beta_{0}\left(T^{2}-f\left(S^{1}\right)\right)=2$. Este exemplo mostra que a condição sobre $i_{*}$ no Teorema 1.3.9(2) é essencial.
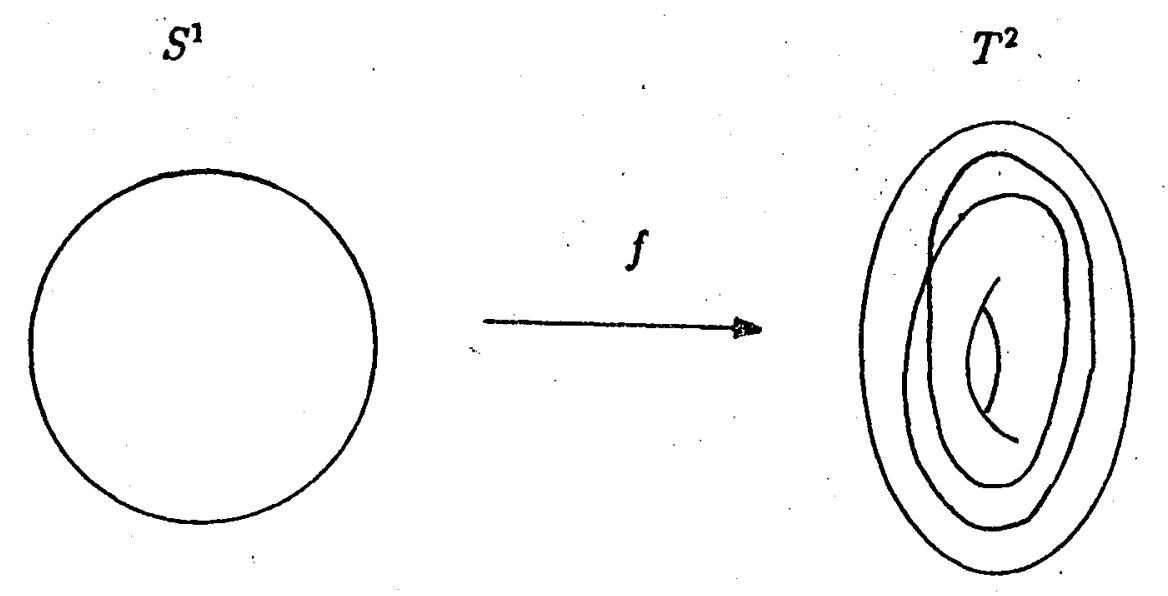

Fig. 1.1

(2) A condição da trivialidade de $\nu(f)$ no Teörema 1.3.9(2) é essencial como pode ser vista através da imersão "standard" com interseções normais da garrafa de Klein em $\mathbb{R}^{3}$.

Como uma consequência imediata do Teorema 1.3.9(1) nós temos uma resposta ao Problema 1.1.2 dada pelo,

Corolário 1.3.11. Seja $M$ uma $(n-1)$-variedade diferenciável, conexa, fechada e orientada, e, seja $f: M \rightarrow S^{n}$ uma imersão com interseções normais. Se $S^{n}-f(M)$ 
tiver exatamente duas componentes conexas, então $f$ é um mergulho.

Mais geralmente, poḍemos apresentar uma caracterização de mergulhos entre imersões com interseçôes normais em codimensão 1, a saber,

Corolário 1.3.12. Sejam $M$ e $N$ variedades diferenciáveis, conexas, fechadas e de dimensões $n-1$ e $n$ respectivamente. Suponhamos que $M$ seja orientada e que $H_{1}(N)=0$. Então, uma imersão com interseções normais $f: M \rightarrow N$ é um mergulho se e somente se $f(M)$ separar $N$ em exatamente duas componentes conexas.

Observações 1.3.13. (1) Substituindo-se a hipótese da orientabilidade de $M$ por $H_{1}(M)=0$ no corolário 1.3.12, Biasi-Fuster (1992) obtem também uma caracterização de mergulhos (ver observação 1.2.6(3)).

(2) Na observação 1.2.6(4), não é necessário assumir que o conjunto de autointerseções de $f$ se reduza ao conjunto dos pontos duplos $S_{2}$ (Podemos também substituir as hipótscs que $H_{1}(M)-H_{1}(N)-0$, estabelecidas por Biaci Fuster (1992), pela nulidade de $i_{*}: H_{n-1}(f(M)) \rightarrow H_{n-1}(N)$ e $j_{*}: H_{n-2}(A) \rightarrow H_{n-2}(M)$, onde $i$ e $j$ são inclusões).

Quando $A=S_{2}$, isto é, quando o conjunto de auto-interseções de $f$ se reduz ao conjunto dos pontos duplos, temos que dim $\operatorname{ker}\left(\left.f\right|_{A}\right)_{*}=\beta_{n-2}(B)=\beta_{0}(B)$. No Teorema 1.3.9, se $A=S_{2}$ e $H_{1}(M)=0$, então $\beta_{0}(N-f(M))=2+\beta_{0}(B)$. Observemos que se $A \neq S_{2}$, então $A$ não é subvariedade de $M$ e poderemos ter situações onde $\operatorname{dim} \operatorname{ker}\left(\left.f\right|_{A}\right)_{*} \neq \beta_{n-2}(B)$. Por exemplo, consideremos a imersão 
com interseções normais $f: S^{2} \rightarrow S^{3}=\mathbb{R}^{3} \cup\{\infty\}$ como na Figura 1.2 abaixo,

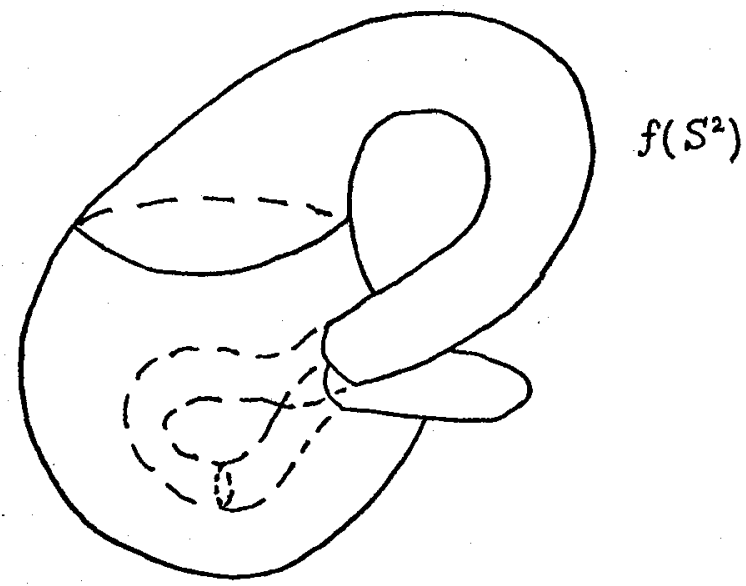

Fig. 1.2

Então, o conjunto de auto-interseção $A$ e sua $f$-imagem $f(A)=B$ são dadas como na Figura 1.3 abaixo. Assim, $\operatorname{dim} \operatorname{ker}\left(\left.f\right|_{A}\right)_{*}=4$ e $\beta_{n-2}(B)=5$. Observemos também que $\beta_{0}\left(S^{3}-f\left(S^{2}\right)\right)=2+\operatorname{dim} \operatorname{ker}\left(\left.f\right|_{A}\right)_{*}=6$.
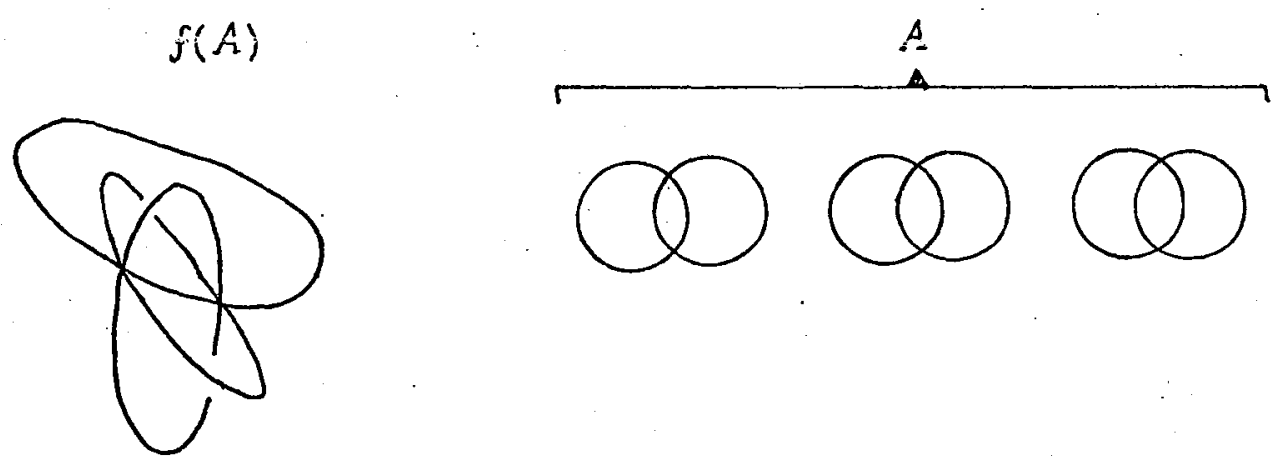

Fig. 1.3 


\section{Capítulo 2}

\section{Observações Topológicas Sobre Certas Aplicações Genéricas Especiais}

\subsection{Problemas Abordados}

Problema 2.1.1. Sejam $f: M^{m} \rightarrow \mathbb{R}^{n}$ uma aplicação genérica especial e $M$ uma variedade diferenciável (classe $C^{\infty}$ ), fechada (compacta e sem bordo) e de dimensão $m(m \geq n)$. Sob que condiçôes é possivel obter informaçōes sobre a topologia de $M$ considerando-se que $m$ e $n$ assumem "valores pequenos"?

Problema 2.1.2. Sejam $f: M^{m} \rightarrow \mathbb{R}^{m-1}$ uma aplicação genérica especial e $M$ uma variedade diferenciável, conexa, fechada e de dimensão $m$. Sob que condições é possível assegurar-se que o conjunto singular de $f, S(f)$, é uma subvariedade nãoenodada em $M$ ?

Problema 2.1.3. Seja $G E\left(M, \mathbb{R}^{2}\right)$ o conjunto das aplicações genéricas especiais de uma $m$-variedade diferenciável e fechada $M$ com valores no plano. Para valores 
"pequenos de $m$ ", pode-se estabelecer alguma classificação para tãis aplicaçōes?

\subsection{Definições e Principais Resultados Relacio- nados}

Seja $M$ uma $m$-variedade diferenciável, conexa e fechada. Uma aplicação diferenciável $f: M^{m} \rightarrow \mathbb{R}^{n}(m \geq n)$ é dita genérica especial se para cada ponto $p \in S(f)$, onde $S(f)$ denota o conjunto singular de $f$, existirem sistemas de coordenadas locais $\left(x_{1}, \ldots, x_{m}\right)$ e $\left(y_{1}, \ldots, y_{n}\right)$ centrados em $p$ e $f(p)$ respectivamentc, através dos quais $f$ se expressa (localmente) por:

$$
\left\{\begin{array} { l } 
{ x _ { 1 } } \\
{ \vdots } \\
{ x _ { m } }
\end{array} \stackrel { \lessgtr } { \rightarrow } \left\{\begin{array}{l}
y_{1}=x_{1} \\
\vdots \\
y_{n-1}=x_{n-1} \\
y_{n}=x_{n}^{2}+\cdots+x_{m}^{2}
\end{array}\right.\right.
$$

Uma ferramenta básica e de utilização essencial para a obtenção da grande mai oria dos resultados relacionados aos Problemas 2.1.1, 2.1.2 e 2.1.3 è a "Fatorização de Stein associada a aplicação dada", a qual é definida a seguir:

Consideremos $f: M^{m} \rightarrow \mathbb{R}^{n}$ genérica especial $(m>n) ; f$ induz em $M$ uma relação de equivalência definida por:

dados $p, p^{\prime} \in M, p \sim p^{\prime}$ se e somente se $f(p)=f\left(p^{\prime}\right)=r$ e $p, p^{\prime}$ estiverem na mesma componente conexa de $f^{-1}(r)$.

0 quociente $M / \sim$ denotamos por $W_{f}$ e a aplicaçâo quociente $M \rightarrow W_{f}$ denotamos por $q_{f}$ (ou simplesmente $q$ ). A comutatividade do diagrama a seguir define $\bar{f}: W_{f} \rightarrow \mathbb{R}^{n}:$ 


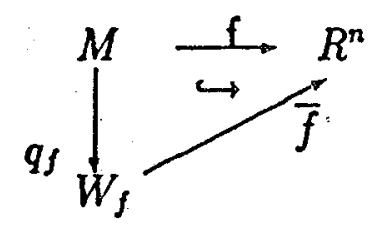

Assim, chamamos $\left(W_{f}, q_{f}, \bar{f}\right)$, ou simplesmente $W_{f}$, de fatorização de Stein associada a $f$

Observaçôes 2.2.1. (1) Quando $m=n$, a fatorização de Stein de $f$ é a própria variedade $M$, não trazendo assim qualquer informação sobre $f$ ou $M$.

(2) Seja $f: M^{m} \rightarrow \mathbb{R}^{n}$ uma aplicação genérica especial $(m>n)$. Analogamente ao estudo que Porto-Furuya (1990) desenvolvem para as dimensões $(m, 2)$, pode-se também introduzir estrutura diferenciável sobre $W_{f}-q(S(f))$ via o homeomorfismo local $\bar{f}: W_{f}-q(S(f)) \rightarrow \mathbb{R}^{n}$, e, estender tal estrutura para todo $W_{f}$, utilizando-se para tanto cartas locais $\left(q(K),\left.\bar{f}\right|_{q(K)}\right)$, onde $K=\left\{\left(x_{1}, \ldots, x_{m}\right) / x_{1}^{2}+\ldots+x_{m}^{2}<\xi^{2}\right\}$ é vizinhança de um ponto arbitrário $p \in S(f)$. Tal estrutura torna $W_{f}$ uma $n$ variedade diferenciável, compacta, orientada e com bordo difeomorfo a $S(f)$, sendo que $\bar{f}: W_{f} \rightarrow \mathbb{R} \mathbb{R}^{\bar{a}}$ e uma imersáo a quai é obtida como extensão da imersão $\left.\bar{f}\right|_{W_{f}-q(S(f))}$. Além disso, $q_{f}: S(f) \rightarrow \partial W_{f}$ é um difeomorfismo, onde $\partial X$ denota bordo da variedade $X$.

Os trabalhos de Burlet-de Rham (1974), Porto-Furuya (1990) e Saeki (1992) consideram o Problema 2.1.1. Inicialmente, trabalhando com aplicações genéricas especiais de 3-variedades diferenciáveis e fechadas com valores no plano tem-se o seguinte resultado:

Teorema 2.2.2 (Burlet-de Rham (1974)). Uma 3-variedade diferenciável e fechada $M$ admite uma aplicação genérica especial em $\mathbb{R}^{2}$ se e somente se $M$ for difeomorfa a uma das seguintes variedades: $V_{r}(r=0,1, \ldots) \propto V_{r}^{*}(r=1,2, \ldots)$, sendo que 
$V_{0}=S^{3}, V_{1}=S^{1} \times S^{2}, V_{r}=\#^{r} V_{1}, V_{1}^{*}=S^{1} \tilde{x} S^{2}$ (isto é, o $S^{2}$-fibrado não trivial sobre $S^{1}$ ) e $V_{r}^{*}=V_{r-1} \# V_{1}^{*}$, onde \# denota soma conexa.

Posteriormente, trabalhando com aplicações genéricas especiais de uma $m$-variedade diferenciável e fechada $M(m>3)$ com valores no plano, Porto-Furuya (1990) estabelecem o resultado:

Teorema 2.2.3 (Porto-Furuya (1990)). Seja $M$ uma $m$-variedade diferenciável e fechada ( $m \geq 3$ ), a qual admite uma aplicação genérica especial em $\mathbb{R}^{2}$, sendo que $\operatorname{rank} \pi_{1}(M)=r$.

(1) Se $r=0$ e $m \geq 3$, então $M$ é homeomorfa a $S^{m}$ (qunando $m=3,4,5$, ou 6 tem-se que $M$ é difeomorfa a $S^{m}$ );

(2) Se $r=1$, então $M$ é homotópica a um $S^{m-1}$-fibrado sobre $S^{2}$ (quando $M$ for orientada e $m=3,4$ ou 6 tem-se que $M$ é difeomorfa a $\left.S^{1} \times S^{m-1}\right)$;

(3) Se $r>1$ sendo $M$ orientada e $m=3,4$, ou 6 , então $M \cong \#^{r} S^{1} \times S^{m-1}$.

Mais recentemente, Saeki (1992) estabelece resultados mais gerais envolvendo os teoremas acima expostos, apresentando também "teoremas de classificação" envolvendo certas aplicações genéricas especiais com valores em $\mathbb{R}^{3} \mathrm{e} e m \mathbb{R}^{2}$. Dentre os principais resultados obtidos por Saeki citamos os teoremas enunciados a seguir.

Teorema 2.2.4 (Saeki (1992)). Uma $m$-variedade diferenciável e fechada $M$ ( $m \geq$ 3) admite uma aplicação genérica especial em $\mathbb{R}^{2}$ se e somente se $M$ for difeomorfa a uma das seguintes variedades: 
(1) se $m \leq 6$,

$$
\begin{array}{lc}
\#^{r} S^{1} \times S^{m-1} & (r \geq 0) \\
\left(\#^{r-1} S^{1} \times S^{m-1}\right) & \#\left(S^{1} \tilde{\times} S^{m-1}\right) \quad(r \geq 1)
\end{array}
$$

(2) se $m \geq 7$,

$$
\begin{aligned}
& \left(\#_{i=1}^{r} S^{1} \times \sum_{i}^{m-1}\right) \# \Sigma^{m} \quad(r \geq 0) \\
& \left(\#_{i=1}^{r-1} S^{1} \times \sum_{i}^{m-1}\right) \#\left(S^{1} \tilde{x} \sum_{r}^{m-1}\right) \# \Sigma^{m} \quad(r \geq 1)
\end{aligned}
$$

onde \# denota soma conexa, $S^{\mathbb{1}} \tilde{x} X$ denota $X$-flbrado não trivial sobre $S^{1}, \Sigma^{m} \in$ $\theta_{m}$ (grupo das classes de h-cobordismo de $m$-esferas homotópicas), $\sum_{i}^{m-1} \in \theta_{m-1}$, $r=\operatorname{rank} \pi_{1}(M)$ e a soma conexa sobre um conjunto vazio ć assumida ser $S^{m}$.

Teorema 2.2.5 (Saeki (1992)). Seja $M$ uma 4-variedade diferenciável, fechada e 1-conexa. Então, $M$ admite uma aplicação genérica especial em $\mathbb{R}^{3}$ se e somente se $M$ for difeomorfa a uma das seguintes variedades:

$$
\begin{aligned}
& \left(\#^{r} S^{2} \times S^{2}\right) \# \sum_{(r \geq 0)}(r \geq 0) \\
& \left(\#^{r-1} S^{z} \times S^{2}\right) \#\left(S^{2} \times S^{2}\right) \# \Sigma \quad(r \geq 1)
\end{aligned}
$$

onde $S^{2} \tilde{x} S^{2}$ denota o $S^{2}$-fibrado não-trivial sobre $S^{2}, \sum$ é uma 4-esfera homotópica e $r=\operatorname{rank} \pi_{1}(M)$.

Teorema 2.2.6 (Saeki (1992)). Uma superfície fechada $M$ admite uma aplicação genérica especial em $\mathbb{R}^{2}$ se e somente se $\chi(M) \equiv 0(\bmod 2)$, onde $\chi$ denota a caracteristica de Euler de $M$.

Burlet-de Rham (1974) consideram o Problema 2.1.2. Assumindo que $M=S^{3}$ e utilizando o lema de Dehn-Papakyriakopoulos eles obtem o não-enodamento do 
conjunto singular em $S^{3}$, mais especificamente,

Teorema 2.2.7 (Burlet-de Rham (1974)). Seja $f: S^{3} \rightarrow \mathbb{R}^{2}$ uma aplicaçâo genérica especial. Então, o conjunto singular de $f, S(f)$, é um círculo não-enodado em $S^{3}$.

Burlet-de Rham (1974) estabelecem também alguns resultados relacionados agora ao Problema 2.1.3. Assumindo $M$ uma 3-variedade diferenciável e fechada, eles introduzem uma relação de equivalência em $G E\left(M^{3}, \mathbb{R}^{2}\right)$ definida por: sejam $f, g \in$ $G\left(M^{3}, \mathbb{R}^{2}\right)$

$$
f \sim g \Leftrightarrow \text { existem difeomorfismos } H: M^{3} \rightarrow M^{3} \text { e } h: W_{f} \rightarrow W_{g} \text { comutando }
$$
o diagrama abaixo

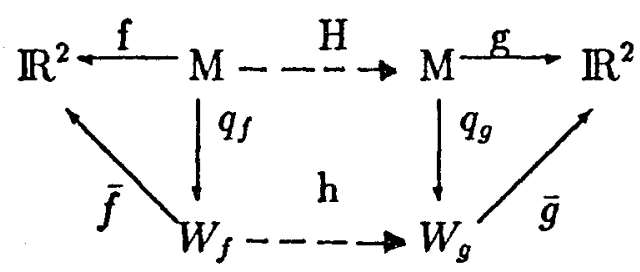

com tal relação Burlet-de Rham (1974) estabelecem uma interessante classificação no conjunto $G E\left(M^{3}, \mathbb{R}^{2}\right)$, a saber:

Teorema 2.2.8 (Burlet-de Rham (1974)). Mantendo-se as notações do teorema 2.2.2 tem-se que,

(1) As aplicações genéricas especiais de $V_{r}$ em $\mathbb{R}^{2}$ se dividem em $\left[\frac{r}{2}\right]+1$ classes distintas correspondentes aos inteiros $b$ que satisfazem as condições
(i)
$b=r+1-2 g$
e $1 \leq b \leq r+1$

(2) Seja o sistema formado pelas condições (i) acima e por: 
(ii) $\left\{\begin{array}{l}1 \leq c \leq b / 2, \text { se } g=0 \\ 0 \leq c \leq b / 2, \text { se } g>0\end{array}\right.$

Então, para cada par de inteiros $b$ e $c$ satisfazendo (i) e (ii), corresponde uma classe de aplicações genéricas especiais de $V_{r}^{*}$ em $\mathbb{R}^{2}$ e reciprocamente; $b$ é o número de componentes do conjunto singular de aplicações na classe e $2 c$ é o número de tais curvas que não possuem vizinhança orientável.

Mais recentemente, Furuya (1986) introduz uma nova relação de equivalência, denominada "imagem-equivalência", no conjunto $G E\left(M, \mathbb{R}^{2}\right)$ assumindo $M$ ser uma $\mathrm{m}$-variedade diferenciável $(m=3,4)$, fechada e orientada. Tal relação é definida como segue:

Sejam $f, g \in G E\left(M, \mathbb{R}^{2}\right), f \equiv g \Leftrightarrow(a) f \sim g$ são equivalentes segundo Burlet-de Rham(1974);

(b) $\bar{f} \equiv \bar{g}$ oh, isto é, existe um difeomorfismo positivo $h_{\imath}: W_{f} \rightarrow W_{g}$ tal que as imersões $\bar{f}$ $e \bar{g}$ oh são imagem-homopóticas, onde $\bar{f} e \bar{g}$ são as respectivas imersões induzidas por íg.

Observação 2.2.9. Seja W uma superfície compacta, conexa e orientada, e $\phi: W \rightarrow$ $\mathbb{R}^{2}$ uma imcrsão; $\phi$ é dita "positiva" se a orientação de $W$ induzir em $\mathbb{R}^{2}$, através de $\phi$, a orientação canônica. Assim, duas imersões positivas $\phi_{0}, \phi_{1}: W \rightarrow \mathbb{R}^{2}$ são ditas "imagem-homotópicas", $\phi_{0} \equiv \phi_{1}$, se existir um difeomorfismo positivo (preservando orientação) $h_{+}: W \rightarrow W$ tal que $\phi_{0}$ e $\phi_{1}$ oh sejam regularmente-homotópicos, ou seja, existe uma homotopia diferenciável $H: W \times[0,1] \rightarrow \mathbb{R}^{2}$ satisfazendo: $H(x, 0)=$ $H_{0}(x)=\phi_{0}(x) ; H(x, 1)=H_{1}(x)=\phi_{1} o h(x)$, e, $H_{t}: W \rightarrow \mathbb{R}^{2}$ é imersão, $\forall t \in[0,1]$, : onde $H_{t}(x)=H(x, t)$.

Com o objetivo de simplificar a definição de "imagem-equivalência", Furuya 
(1986) demonstra que:

Teorema 2.2.10 (Furuya (1986)). Seja $M$ uma $m$-variedade diferenciável, fechada e orientada, com $m=3$ ou 4 . Então,

$f, g \in G E\left(M, \mathbb{R}^{2}\right)$ são imagem equivalentes se, e somente se, existir um difeomorfismo positivo $H_{+}: M \rightarrow M$ e uma família diferenciável $\left(f_{t}\right)$ de aplicações genéricas especiais, com $t \in[0,1]$, tal que $f_{0}=f$ e $f_{1}=g o H_{+}$.

Observação 2.2.11. Furuya (1986) observa que esta nova relação de equivalência decompõe as classes segundo a relação de Burlet-de Rham (1974) em subclasses, em correspondência com as classes de imagem-homotopia de imersões. Como exemplo Furuya (1986) observa que as duas aplicações cujas imagens são dadas pelas Figuras 2.1 e 2.2 abaixo, são equivalentes segundo Burlet-de Rham (1974), porém não são imagem-equivalentes.

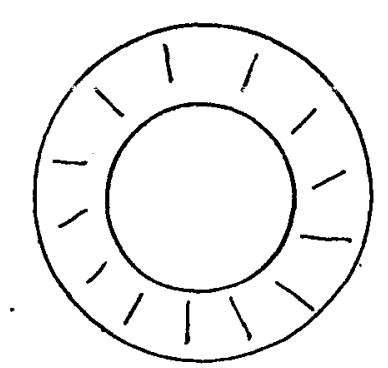

Fig. 2.1

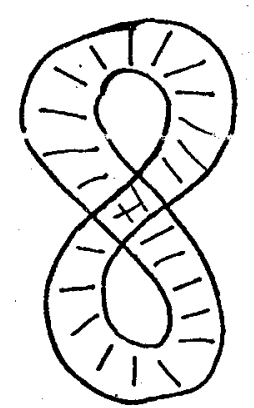

Fig. 2.2.

\subsection{Resultados Obtidos}

No que segue, $M$ denota uma $m$-variedade diferenciável (classe $C^{\infty}$ ), conexa e fechada (compacta e sem bordo) e $f: M \rightarrow \mathbb{R}^{n}(m \geq n)$ denota uma aplicação 
genérica especial.

Observaçẫo 2.3.1. Quando $n=1$, pela definição de aplicações genéricas especiais, tem-se que tais aplicaçôes são simplesmente aplicações de Morse com um ponto crítico de mínimo e outro de máximo. Neste caso, pelo teorema de Reeb (ver Hirsch (1976)), tem-se que $M$ é homeomorfa a $S^{m}$. Assim, para certos valores pequenos de $m, M$ é não exótica (ver Kervaire-Milnor (1963)). Portanto, aplicações genéricas especiais nestas condições estão completamente caracterizadas.

Tomando $f: M^{m} \rightarrow \mathbb{R}^{2}(m>2)$ genérica especial, Porto-Furuya (1990), obtem o isomorfismo entre $\pi_{1}(M)$ e $\pi_{1}\left(W_{f}\right)$. Este resultado pode ser generalizado para dimensões maiores como segue:

Lema 2.3.2. Seja $f: M^{m} \rightarrow \mathbb{R}^{n}(m>n+1)$ genérica especial. Então, $q_{*}: \pi_{1}(M) \rightarrow$ $\pi_{1}\left(W_{f}\right)$ é isomorfismo.

\section{Prova:}

Serão utilizados argumentos similares aos desenvolvidos por Porto-Furuya (1990). Inicialmente, considera-se o fibrado

$$
\begin{gathered}
S^{m-n} \hookrightarrow M-S(f) \\
\left.\stackrel{\downarrow}{\mathrm{q}}\right|_{w_{j}^{\circ}} \\
W_{j}
\end{gathered}
$$

onde $\left.q\right|_{W_{j}^{\circ}}$ é submersão e $W_{f}^{o}$ denota o interior de $W_{f}$. Tomando-se um ponto base $a \in M-S(f)$ e sendo $b=q(a)$, pela sequência de homologia do fibrado acima, temse o isomorfismo $\left.q\right|_{w_{f_{0}}:} \pi_{1}(M-S(f), a) \rightarrow \pi_{1}\left(W_{f}^{o}, b\right)$. Naturalmente, $\pi_{1}\left(W_{f}^{o}, b\right)$ é isomorfo e $\pi_{1}\left(W_{f}, b\right)$. Considerando-se $j: M-S(f) \rightarrow M$ a aplicação inclusão, então é suficiente demonstrar-se que $j_{*}$ é isomorfismo. De fato:

(1) $j_{*}$ é sobrejetor:

Seja $\gamma: S^{\prime} \rightarrow M$ um laço com ponto base a. Como $\operatorname{dim} S(f)=n-1$, podemos deformar $\gamma$ homotopicamente, desviando $\gamma$ de $S(f)$, conforme a Figura 2.3 
abaixo:

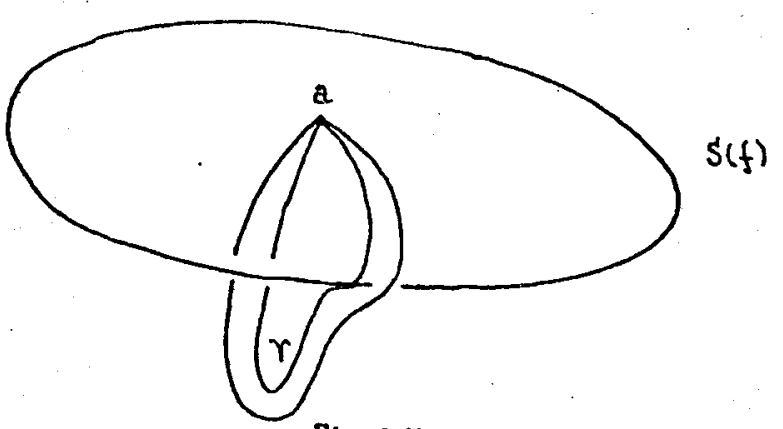

(2) j* é injetor:

fig. 2.3

Suponhamos que existam laços $\gamma_{1}$ e $\gamma_{2}$ com ponto base a tais que $\gamma_{i} \sim a$ em $M$, conforme as Figuras 2.4 e 2.5 abaixo:

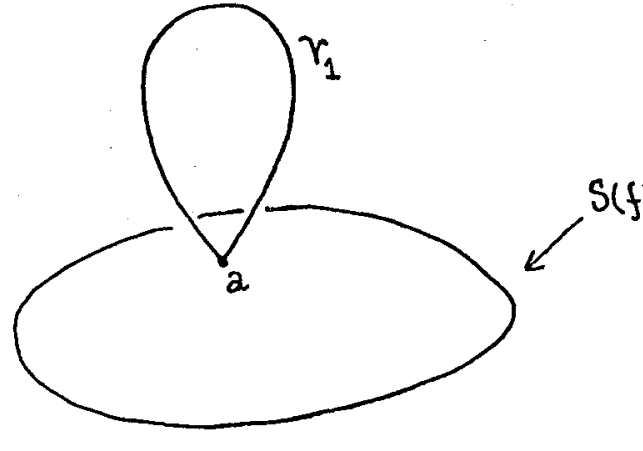

Fig. 2.4

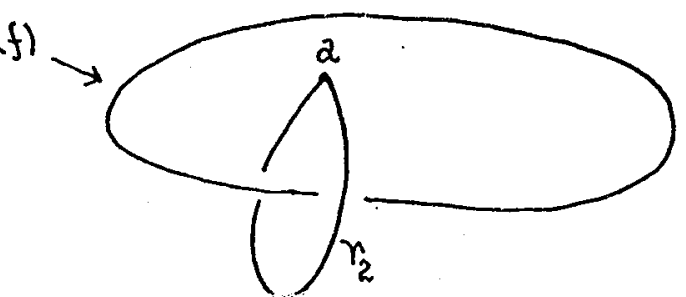

Fig. 2.5

Assim, é suficiente demonstrar-se que o laço descrito na Figura 2.5 acima não ocorre. Seja $\phi$ extensão de $\gamma_{2}$ ao 2-disco $D^{2}, \phi: D^{2} \rightarrow M, \phi \in C^{\infty}$. Usando o teorema da transversalidade de Thom, pode-se supor, sem perda de generalidade, que $\phi$ é transversal a $S(f)$. Logo, $\operatorname{dim} S(f)+\operatorname{dim} D^{2}<\operatorname{dim} M$, o que implica $\phi\left(D^{2}\right) \cap S(f)=\emptyset$, ou seja, não existe laço nas condições descritas na Figura 2.5 .

Quando $m=n+1$ também pode-se provar que $q_{*}$ é isomorfismo(Hiratuka (1992)) Fukuda (1987) estabelece o seguinte resultado:

Lema 2.3.3 (Fukuda (1987)). Seja $f: M^{m} \rightarrow \mathbb{R}^{n}$ uma aplicação genérica especial, com $m>n$. Então, 
(1) $\chi(M) \equiv \chi(S(f)) \bmod 2$;

(2) Se $m-n+1$ é par, então $\chi(M)=\chi(S(f))$, onde $\chi(N)$ denota a característica de Euler do espaço $N$.

Observações 2.3.4. (a) Nesta mesma linha, Saeki (1992) mostra que se $m$ e $n$ forem pares, então $\chi(M)=2 \chi\left(W_{f}\right)$.

(b) Tomando-se $n=2$ e $m$ par, pelo lema 2.3.3(1) segue-se que $\chi(M)$ é par. Nestas dimensões, e sendo $M$ orientada, Levine (1966) obtem uma estimativa mais precisa para $\chi(M)$, a saber: $\chi(M)=\sum_{c} r(c)$, onde $S(f)$ tem orientação bem definida, $c$ percorre as componentes conexas de $S(f)$, sendo $r(c)$ o grau da aplicação $\phi$ restrita a $c$, onde $\phi: S(f) \rightarrow \mathbb{R} P^{1}$ é tal que $\phi(x)$ é a reta passando pela origem de $\mathbb{R}^{2}$, paralela à tangente de $f(S(f))$ em $f(x)$.

(c) Tomando-se $m$ e $n$ pares, novamente o lema 2.3.3(1) apenas nos diz que $\chi(M)$ é par. Nestas dimensões e supondo $M$ orientada, Kushner (1982) estabelece uma estimativa mais precisa para $\chi(M)$, a saber: $\chi(M)=\sum_{\hat{c}} r(\hat{c})$, onde $r(\hat{c})$ é o grau de uma certa aplicação $\left.\hat{\phi}\right|_{\hat{c}}$ e $\hat{c}$ percorre as componentes do conjunto singular de uma certa aplicação $\hat{d} f$ construída por Kushner (1982).

Considerando-se $f$ como no lema 2.3 .3 e assumindo que $n$ seja impar, pelo fato de $S(f)$ ser difeomorfo ao bordo de $W_{f}$, tem-se que $\operatorname{dim} S(f)=n-1$ (par). Assim, tomando-se $n=3$, o teorema de classificação de superfícies nos permite obter algumas interessantes informações sobre $S(f)$, e consequentemente sobre $M$, a saber:

Teorema 2.3.5. Seja $f: M^{m} \rightarrow \mathbb{R}^{3}$ uma aplicação genérica especial com $m>3$. Se $M$ for 1-conexa, então $S(f)$ é uma união finita e disjunta de 2-esferas.

Prova:

Pelo lema 2.3.2 tem-se que $q_{*}: \pi_{1}(M) \rightarrow \pi_{1}\left(W_{f}\right)$ é isomorfismo. Assim, como $M$ é 1-conexa, segue-se que $W_{f}$ também é 1-conexa. Então, $H_{1}\left(W_{f}, Z\right)=0$ e, pelo 
teorema dos coeficientes universais, tem-se que $H^{1}\left(W_{f}, Z\right)=0$.

Tomando-se a sequência exata de homologia do par $\left(W_{f}, \partial W_{f}\right)$ segue que:

$$
\begin{aligned}
& \ldots \rightarrow H_{2}\left(W_{f}, Z\right) \rightarrow H_{2}\left(W_{f}, \partial W_{f}, Z\right) \rightarrow H_{1}\left(\partial W_{f}, Z\right) \rightarrow H_{1}\left(W_{f}, Z\right) \rightarrow \ldots \\
& \text { II } \\
& \text { dualidade } 0 \\
& H^{1}\left(W_{f}, Z\right) \\
& \text { II } \\
& 0
\end{aligned}
$$

isto é, $H_{1}\left(\partial W_{f}, Z\right)=0$. Uma vez que $S(f)$ é difeomorfo a $\partial W_{f}$, tem-se que $S(f)$ é uma superfície, com $H_{1}(S(f), Z)=0$, ou seja, $S(f)=U S^{2}$.

Corolário 2.3.6. Seja $f: M^{m} \rightarrow \mathbb{R}^{3}$ uma aplicação genérica especial, $m>3, m$ par. Se $M$ for 1-conexa, entãa $\chi(M)=2$. $\left(\# C_{S(f)}\right)$, onde $\# C_{S(f)}$ denota o número de componentes conexas de $S(f)$.

\section{Prova:}

Por 2.3.3(2), temos que $\chi(M)=\chi(S(f))$. Assim, pelo teorema 2.3.5 segue-se o resultado desejado.

Corolário 2.3.7. Seja $f: M^{m} \rightarrow \mathbb{R}^{3}$ uma aplicação genérica especial com $m=4$ e seja $M$ 1-conexa. Se $W_{f}$ for difeomorfa ao 3-disco, então $M$ é difeomorfa a $S^{4}$.

\section{Prova:}

Como $S(f) \cong \partial W_{f}$ e pelo corolário acima $\chi(M)=2 .\left(\# C_{S(f)}\right)$ tem-se que $\chi(M)=2$. Logo, $M$ tem homologia de $S^{4}$, e, pela conjectura de. Poincaré 4dimensional (ver Freedman (1982)) segue-se que $M$ é homeomorfa a $S^{4}$. Agora, devido a unicidade da estrutura diferenciável de $S^{4}$, segue-se o resultado.

Corolário 2.3.8. Seja $f: M^{m} \rightarrow \mathbb{R}^{3}$ uma aplicação genérica especial com $m=6$ 
e seja $M$ 1-conexa. Se a homologia média de $M$ for nula e $W_{f}$ for difeomorfa ao 3-disco, então $M$ é difeomorfa a $S^{6}$.

\section{Prova:}

Utilizando-se os argumentos expostos acima, tem-se que $\chi(M)=2$. Logo, por dualidade e pelas hipóteses sobre $M$, segue-se que $M$ tem homologia de $S^{6}$. Portanto, pela conjectura de Poincaré generalizada (ver Smale (1956)), segue-se quẹ $M$ é difeomorfa a $S^{6}$.

Observação 2.3.9: Utilizando argumentos expostos acima, pode-se obter uma prova alternativa ao teorema 2.2.3.(1) quando $m=4$. De fato: 'como $M$ é 1-conexa, pelo lema 2.3.2 e a observação 2.3.4(a), tem-se que $\chi(M)=2$. Logo, por dualidade, seguese que $M$ tem homologia de $S^{4}$. Assim, pela conjectura de Poincaré 4-dimensional e pela unicidade da estrutura diferenciável de $S^{4}$ segue-se que $M$ é difeomorfa a $S^{4}$.

Como já haviamos observado anteriormente, se $M^{m}$ admite uma aplicação $f$ que é genérica especial e assume valores em $\mathbb{R}^{m}$, a sua fatorização de Stein em "nada auxilia no estudo da topologia de $M$ ". Contudo, considerando-se uma situação parificular onủe $m=4 \mathrm{e}$, se aiém das hipóieses gerais sobre $\grave{M}$, assume-se ainda que $M$ seja 1-conexa , então é possivel estabelecer uma interessante informação sobre a topologia de $M$. Para tanto, observe-se inicialmente que, como $H_{1}(M, Z)=0$ pelo teorema dos coeficientes universais, tem-se que $H^{2}(M, Z)$ é livre de torção, ou seja, Tor $\left(H^{2}(M, Z)\right)=0$. Assim, construimos uma forma bilinear simétrica $\omega$ como segue: $\omega: H^{2}(M, Z) \otimes H^{2}(M, Z) \rightarrow Z$ é definida por $\omega(a, b)=\left\langle a \cup b,[M]>\right.$, onde $[M] \in H_{4}(M, Z)$ é a classe fundamental de $M$ e $U$ denota o produto cup. Pela dualidade de Poincaré identificamos $H^{2}(M, Z)$ com $H_{2}(M, Z)$. Logo, em homologia, para $x, y \in H_{2}(M, Z)$ pode-se "descrever geometricamente" $\omega(x, y)$ como o número de interseções de superfícies fechadas e orientadas $X$ e $Y$ em $M$, as quais se interceptam transversalmente e representam $x$ e $y$ respectivamente (ver Dubrovin et alli (1990)). Tem-se que $\omega$ é unimodular, isto é, qualquer matriz representando $\omega$ tem determinante \pm 1 (ver Milnor (1958)). Diz-se que $\omega$ é "par" se $\omega(a, a) \in 2 Z$ para todo $a \in H^{2}(M, Z)$. 
Agora, combinando-se os importantes resultados: Teorema de Classificação de Freedman (ver Freedman (1982)) e o Teorema de Donaldson (ver Donaldson (1983)), pode-se estabelecer como consequência que:

Lema 2.3.10 (Freedman (1982) - Donaldson (1983)).

Seja $M$ uma 4-variedade diferenciável, fechada e 1-conexa. Se a forma $\omega$ associada a $M$ for par e definida positiva, então $M$ é homeomorfa a $S^{4}$.

Recordemos a seguir um resultado obtido por Èliăsberg (1970):

Lema 2.3.11 (Èliăsberg (1970))

Seja $N$ uma $n$-variedade diferenciável, fechada e orientada. Então, $N$ admite uma aplicação genérica especial em $\mathbb{R}^{n}$ se e somente se $M$ for estavelmente paralelizável.

Cunivinanúu-se us leiriás $2.2 .10=2.2 .11$, pode se obter uma interessante informação sobre a topologia de $M$, no caso de $M$ ser 4 dimensional e domínio de uma aplicação genérica especial, com valores em $\mathbb{R}^{4}$.

Teorema 2.3.12. Sejam $f: M^{4} \rightarrow \mathbb{R}^{4}$ uma aplicação genérica especial e $M$ uma 4-variedade diferenciável, fechada, 1-conexa . Se a forma $\omega$ associada a $M$ for definida positiva, então $M$ é difeomorfa a $S^{4}$.

Prova:

Inicialmente observa-se que $\omega$ é par se e somente se a segunda classe de StiefelWhitney de $M, \omega_{2}(M)$, for nula (ver Freed-Uhlenbeck (1984)).

Por outro lado, por 2.3.11, tem-se que $T M \oplus \epsilon^{1}$ é trivial, onde $\epsilon^{1}$ é o fibrado em linha trivial sobre $M$. Assim, $\omega_{2}(M)=\omega_{2}\left(T M \oplus \epsilon^{1}\right)=0$. Portanto, por 2.3.10, 
tem-se que $M$ é homeomorfa a $S^{4}$. Logo, pela unicidade da estrutura diferenciável de $S^{4}$, segue o resultado.

Assim, como resposta ao Problema 2.1.1, temos a observação 2.3.1, os corolários 2.3.7 e 2.3.8, e, o teorema 2.3.12.

Quanto ao Problema 2.1.2, apresentamos dois interessantes resultados caracterizando o não-enodamento do conjunto singular na variedade domínio de certas aplicações genéricas especiais, a saber:

Teorema 2.3.13. Seja $f: M^{m} \rightarrow \mathbb{R}^{m-1}$ uma aplicação genérica especial cujo conjunto singular $S(f)$ assumimos ser uma $(m-2)$-esfera homotópica $(m \neq 5,6)$ ou uma $(m-2)$-esfera $(m=5,6)$. Se $W_{f}$ for contratil $(m \neq 4)$ ou um $(m-1)$-disco $(m=4)$, então $S(f)$ é não-enodada em $M$.

\section{Prova:}

Suponhamos inicialmente $m \geq 7$. Como $W_{f}$ é uma $(m-1)$-variedade diferenciável, contratil, compacta, 1-conexa e com bordo difeomorfo a $S(f)$, o qual também 1-conexo, segue-se pela "caracterização diferenciável de discos" (ver proposição (A), pág. 108 em Milnor (1965)), que $W_{f}$ é difeomorfa ao $(m-1)$-disco.

Agora, se $m=6$, então pela "caracterização diferenciável de 5-discos" (ver proposição (c), pág. 110 em Milnor (1965)), tem-se que $W_{f}$ é difeomorfo ao 5-disco. Porém, quando $m=5$, não é possível demonstrar que $W_{f}$ é difeomorfa a $D^{4}$.

Finalmente, se $m=2$ ou 3 é natural, pelos teoremas de classificação nas referidas dimensões, que $W_{f}$ seja um $(m-1)$-disco.

Por outro lado, como a diferença entre as dimensões de $M$ e $\mathbb{R}^{m-1}$ é igual a 1 , então existe um $D^{2}$-fibrado $\tilde{E}$ sobre $W_{f}$ tal que $M$ seja difeomorfo a $\partial \tilde{E}$ e $S(f)$ seja difeomorfo a $\partial\left(W_{f} \times\{0\}\right)$ (ver Saeki (1992) - proposição 3.4). Como $W_{f}$ é contratil, segue-se que $\partial \tilde{E}$ é difeomorfo a $\partial\left(W_{f} \times D^{2}\right)$. Portanto, para $m \neq 5$, tem-se que $S(f)$ é uma $(m-2)$-esfera não-enodada em $M \cong S^{m}$. Finalmente, quando $m=5$, como por hipótese $W_{f}$ é contratil, tem-se que $M$ é uma 5-esfera homotópica (ver pro- 
posição 4.1 em Saeki (1992)). Assim, pela conjectura generalizada de Poincaré (ver Smale (1956)), tem-se que $M$ é difeomorfa a $S^{5}$. Agora, por hipótese $S(f) \cong S^{3}$, e, então $M-S(f) \cong S^{5}-S^{3}$ é um $S^{1}$-fibrado sobre o interior $W_{f}, W_{f}^{o}$. Logo, tomando-se a sequência de homotopia associada e este fibrado, tem-se ( $W_{f}$ é contratiij) naturalmente que $S^{5}-S^{3}$ é umạ equivalência de homotopia com $S^{1}$. Portanto, por Levine (1970), segue-se que $S^{3}$ é não-enodado em $S^{5}$.

Corolário 2.3.14. Seja $f: M^{m} \rightarrow \mathbb{R}^{m-1}$ uma aplicação genérica especial tal que $S(f)$ seja uma $(m-2)$-esfera homotópica $(m \neq 5,6)$ ou $(m-2)$-esfera $(m=5,6)$. Se $\beta_{0}\left(\mathbb{R}^{m-1}-f(S(f))\right)=2$, então $S(f)$ é não-enodado em $M$, onde $\beta_{k}(X)$ denota o k-ésimo número de Betti de $X$.

\section{Prova:}

Como $f$ é genérica especial, e, portanto é uma particular aplicação estável, temse que $\left.f\right|_{s(f)}: S(f) \rightarrow \mathrm{R}^{m-1}$ é uma imersão com interseções normais (ver capítulo anterior). Assim, pelo corolário 1.3 .10 , segue-se que $S(f)$ mergulha $\mathrm{em} \mathbb{R}^{m-1}$ (via $\left.\left.f\right|_{S(f)}\right)$. Agora, como $\left.q\right|_{S(f)}$ é difeomorfismo, tem-se que $\left.\bar{f}\right|_{\partial W_{f}}: \partial W_{f} \rightarrow \mathbb{R}^{m-1}$ é mergulho. Pelo fato de $\bar{f}: W_{f} \rightarrow \mathbb{R}^{m-1}$ ser imersão tem-se que $\bar{f}\left(W_{f}\right)$ é a componente limitada de $\mathbb{R}^{m-1}-\bar{f}\left(\partial W_{f}\right)$, tendo evidentemente como bordo $\bar{f}\left(\partial W_{f}\right)$ o qual é 1-conexo. Utilizando-se o bicolar de $\bar{f}\left(\partial W_{f}\right)$ em $\overline{\mathbb{R}}^{m-1}$ (sua exisiêucia é garantida por (3.6) em Milnor (1965)) e o teorema de Van Kampen tem-se que $\bar{f}\left(W_{f}\right)$ é 1-conexo. Como $\bar{f}\left(W_{f}\right)$ tem homologia de um ponto (via Mayer-Vietovis), tem-se que $\bar{f}\left(W_{f}\right)$ é difeomorfo ao $(m-1)$-disco $(m \geq 6)$ (via caracterizações de discos em Milnor (1965)). Agora, quando $m \leq 5$ tem-se que $\bar{f}\left(W_{f}\right)$ é homeomorfa ao $(m-1)$-disco (ver (9.7) em Milnor (1965)), com bordo difeomorfo a $(m-2)$-esfera. Observemos que para $m=4$ tem-se, via o teorema de classificação de superfícies, que $S(f)$ é difeomorfa a $S^{2}$. Logo, o teorema de Schoenflies generalizado assegura que $\bar{f}\left(W_{f}^{3}\right)$ é difeomorfo a $D^{3}$.

Por outro lado, utilizando-se do teor.de Ehresmann, tem-se que $\bar{f}: W_{f} \rightarrow \bar{f}\left(W_{f}\right)$ é recobrimento de p-folhas. Logo, restringuindo-se ao bordo de $W_{f}$, tem-se que $\mathrm{p}=1$, isto é, $W_{f}$ é difeomorfo a $\bar{f}\left(W_{f}\right)$. Portanto, pelo teorema anterior, segue-se o 
resultado.

Para finalizar este capítulo, procuramos obter informaçôes relacionadas ao Problema 2.1.3.

Consideremos $M$ uma $m$-variedade diferenciável $(m=4,5)$ e fechada. Seja $G E\left(M, \mathbb{R}^{2}\right)$ o conjunto das aplicaçōes genéricas especiais de $M$ em $\mathbb{R}^{2}$. A relação introduzida por Burlet-de Rham (1974) sobre $G E\left(M^{3}, \mathbb{R}^{2}\right)$, com $M$ tridimensional, "generaliza-se" para dimensões maiores de $M$. Em dimensões $m=4,5$, com $M$ orientada, sabe-se que se tais variedades $M$ são domínio de aplicações genéricas com valores no plano, então $M$ é difeomorfo a um dos seguintes tjpos:

$S^{4}, \#^{r} S^{1} \times S^{3}(r \geq 1)$, em dimensão 4 (ver teorema 2.2 .3 );

$S^{5}, \#^{r} S^{1} \times S^{4}(r \geq 1)$, em dimensão 5 (ver teorema 2.2.4). Assim, pode-se generalizar o teorema 2.2 .8 (1) como segue:

Teorema 2.3.15. Suponhamos que $M$ seja uma $m$-variedade diferenciável ( $m=$ $4,5)$, fechada e orientda. Então, o conjunto $G E\left(M, \mathbb{R}^{2}\right)$ munido da relação $\sim$ (extensão natural da relação estabelecida por Burlet-de Rham (1974)), tem o número de classes distintas dado pelo número de inteiros $b$ satisfazendo as condições $b \equiv r+1$ $(\bmod 2)$ e $1 \leq b \leq r+1$, onde $r=\operatorname{rank} \pi_{1}(M)$.

\section{Prova:}

Seja $k \in G E\left(M, \mathbb{R}^{2}\right)$. Então, existe um único $S^{m-2}$-fibrado $M^{\prime}$ sobre $W_{k}$ com grupo estrutural $D$ if $f_{+}\left(S^{m-2}\right)$ (difeomorfismos preservando orientação), tal que $M$ é obtido de $M^{\prime}$ identificando-se os pontos das fibras sobre o bordo de $W_{k}$ (ver PortoFuruya (1990)).

Logo, dados $f, g \in G E\left(M, \mathbb{R}^{2}\right)$, sejam $\left(M^{\prime}, \pi^{\prime}, W_{f}\right)$ e $\left(M^{\prime \prime}, \pi^{\prime \prime}, W_{g}\right)$ os fibrados induzidos por $f$ e $g$ respectivamente. Uma vez que, $\pi_{o}\left(D i f f_{+}\left(S^{m-2}\right)\right)=0(m=$ $4,5)$, tem-se que tais fibrados são triviais. Assim, dado um difeomorfismo $h: W_{f} \rightarrow$ $W_{g}$, este induz um difeomorfismo. $\tilde{H}: W_{f} \times S^{m-2} \rightarrow W_{g} \times S^{m-2}$ satisfazendo a igual- 
dade $h o \pi^{\prime}=\pi^{\prime \prime} \circ \tilde{H}$. Logo, constroi-se um difeomorfismo $H$ comutando o diagrama abaixo:

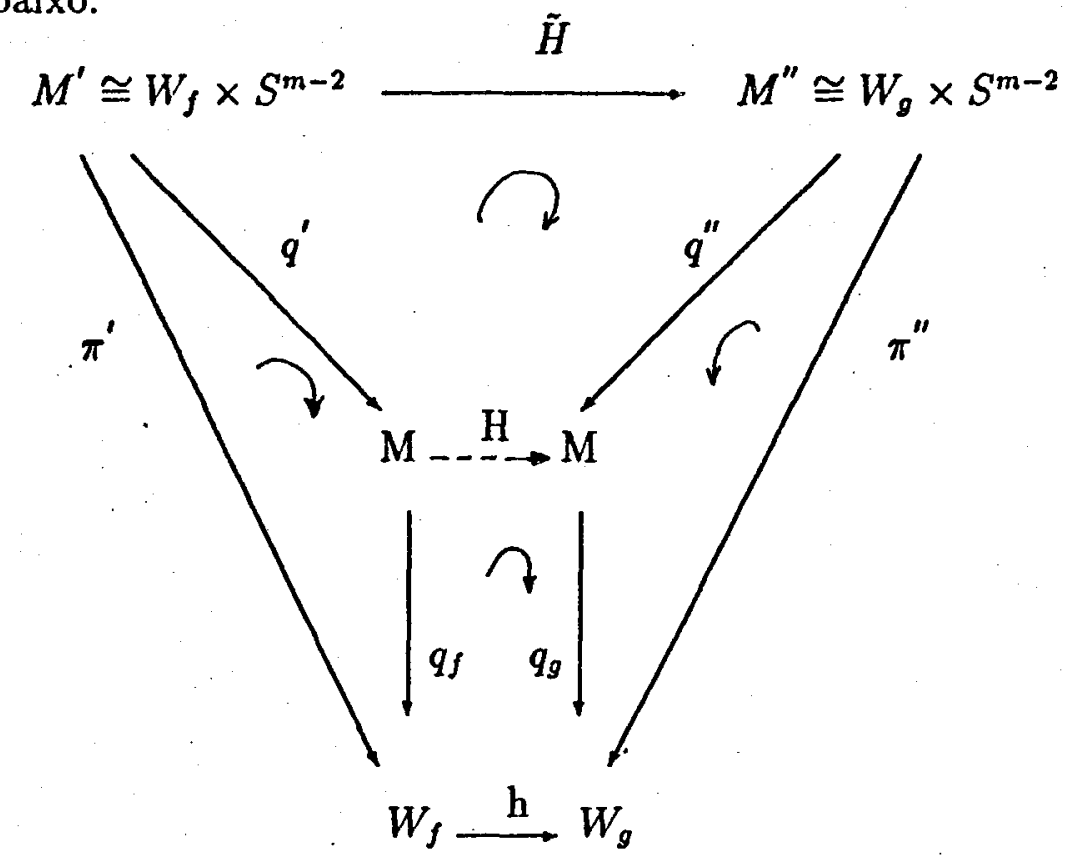

onde $q^{\prime}, q^{\prime \prime}$ são as respectivas projeções de $M^{\prime}$ e $M^{\prime \prime}$ sobre $M$ tais que $q_{f} \circ q^{\prime}=\pi^{\prime}$ e $q_{s} \circ q^{\prime \prime}=\pi^{\prime \prime}$. Portanto, quando $W_{f}$ for difeomorfo a $W_{g}$, as aplicações $f$ e $g$ estão na mesma classe. Assim, o número de classes distintas está em correspondência com o número de superíicies $\dddot{W}_{f}$ nào diîeomorías entre si coun $j \in \mathcal{G} E\left(M i, \mathbb{R}^{2}\right)$. Uma vez que $M \cong \#^{r} S^{1} \times S^{m-1}(n \geq 1)$ ou $M \cong S^{4}$ ou $S^{5}(r=0)$, tem-se que $\operatorname{rank} \pi_{1}(M)=\operatorname{rank} \pi_{1}\left(W_{f}\right)=r=2 g+b-1$, onde $g$ e $b$ representam respectivamente os genus è o número de componentes de bordo de $W_{f}$. Logo, existem "tantos $W_{f}$ não difeomorfos" quantas forem o número de combinações de $g$ e $b$ com $b=(r+1)-2 g$. Isto completa a prova.

Observação 2.3.16. Quando $M$ for não-orientada, pode-se tamiém estender o teorema 2.2.8(2) para dimensões 4 e 5 . Nestas dimensões, utilizando-se do trabalho de Saeki (1992), é possivel caracterizar todas as variedades não-orientadas que são dominio de aplicações genéricas especiais à valores $e m \mathbb{R}^{2}$. Assim, via os resultados (2.1), (2.2) e (2.3) encontrados em Porto-Furuya (1990), e, o fato de $\pi_{o}\left(\operatorname{Diff}_{+}\left(S^{m-2}\right)\right)=0(m=4,5)$, pode-se mostrar (de forma similar ao obtido 
por Burlet-de Rham (1974)) que: "Dada $f \in G E\left(M^{m}, \mathbb{R}^{2}\right)(m=4,5)$ sua classe se corresponde biunivocamente a um revestimento de duas folhas de $W_{f}$ ", o que é essencial para a extensão de 2.2 .8 (2).

Observação 2.3.17. Seja $M$ uma $m$-variedade diferenciável, fechada e orientada onde $\pi_{o}\left(D i f f_{+}\left(S^{m-2}\right)\right)=0$ (por exemplo, $m=5$ ). Então, naturalmente o teorema 2.2.10 nestas condições continua válido. Para tanto, utilizamos o resultado (2.3) em Porto-Furuya (1990) e o fato de que todo $S^{m-2}$-fibrado orientado (grupo estrutural é $D i f f_{+}\left(S^{m-2}\right)$ sobre uma superfície compacta e orientada é trivial ( $m$ nas condições acima).

Como visto anteriormente, a relação $\equiv$ estabelecida por Furuya (1986) refina a relação estabelecida por Burlet-de Rham (1974). Em alguns casos particulares pode-se determinar o número de classes distintas sob tais relações. Para tanto, considerando-se $M^{m}(m=3$, 4ou 5) $m$-variedade diferenciável, fechada e orientada, toma-se $A=\left\{f \in G E\left(M, \mathbb{R}^{2}\right)\right.$ tal que $W_{f} \cong D_{(k)}^{2}$, onde $D_{(k)}^{2}$ representa um 2-disco com $k$-buracos $\}$.

Observação 2.3.18. Seja $W$ uma superfície orientada e compacta, tendo $a_{o}, \ldots, a_{k}$ componentes de bordo, tal que exista $\phi: W \rightarrow \mathbb{R}^{2}$ uma imersão positiva. Cada curva (simples) $a_{i}$ pode ser representada por uma aplicação $\alpha_{i}: S^{1} \rightarrow W$ diferenciável. Assim, define-se o grau de $\phi$ com respeito a $a_{i}, g_{\phi}\left(a_{i}\right)$, como o grau de curva $\phi o \alpha_{i}$ : $S^{1} \rightarrow \mathbb{R}^{2}$ (este grau é invariante por homotopia-regular). Denota-se por $\beta_{k}$ a coleção de conjunto de inteiros $\left\{\eta_{0}, \ldots, \eta_{k}\right\}$ satisfazendo $\sum_{j=0}^{k} \eta_{j}=k-1$. Assim, para $\phi: W \rightarrow \mathbb{R}^{2}$, define-se $\beta(\phi)=\left\{g_{f}\left(a_{c}\right), \ldots, g_{f}\left(a_{k}\right)\right\} \in \beta_{k}$. Em Kauffman (1979) (ver proposição 2.3) tem-se que $\beta($.) é invariante por imagem-homotopia. Logo, se $I(W)$ denota o conjunto de classes de imagem-homotopia de imersões positivas de $W \rightarrow \mathbb{R}^{2}$, tem-se que $I(W) \approx \beta_{k}$.

Assim, considerando-se $A$ munido da relação $\equiv$, tem-se que o número de classes 
distintas fica determinado pelonúmero de elementos $\beta_{k}$, \#㱠. Então, quando $k=0$ uma vez que $\# \beta_{0}=1$, observa-se que as duas relações $(\sim e \equiv)$ coincidem quando a fatorização de Stein correspondente for um 2-disco. Agora, quando $k=1$, segundo a relação $\sim$ existe uma só classe realizando $D_{(1)}^{2}$, contudo desde que $\beta_{1}=N$ (conjunto dos números naturais) (ver Kauffman (1979)), tem-se uma quantidade enumerávèl de subclasses distintas segundo a relação $\equiv$. 


\section{Capítulo 3}

\section{Sobre a Topologia de Variedades Realizando Complexos Bidimensionais}

\subsection{Problemas Abordados}

Problema 3.1.1 Dada uma aplicação estável $f: M^{m} \rightarrow \mathbb{R}^{2}$, esta induz um homomorfismo $q_{f}: \pi_{1}(M) \rightarrow \pi_{1}\left(W_{f}\right)$. Sob quais condições é possivel assegurar-se que $q_{f,}$ é epimorfismo?

Problema 3.1.2 Considere-se $W$ um complexo bidimensional pré-determinado, o qual corresponde à fatorização de Stein de umá $m$-variedade diferenciável $M^{m}$ a valores no plano, cujas únicas singularidades são as dobras. Sob quais condições, em dimensões baixas $(m=3,4)$, é possivel obter informações sobre a topologia da variedade $M$ que realiza o modelo $W$ ? 


\subsection{Definições e Principais Resultados Relacio- nados}

Seja $M$ uma $m$-variedade diferenciável e fechada. Uma aplicação diferenciável $f: M \rightarrow \mathbb{R}^{2}$ é dita "estável" se para cada ponto singular $p$ em $M$ existirem sistemas de coordenadas locais $\left(u, x, y_{1}, \ldots, y_{m-2}\right)$ e $(U, Y)$ centrados em $p$ e $f(p)$ respectivamente, através dos quais $f$ se expressa por:

(i) $\left(u, x, \dot{y}_{1}, \ldots, y_{m-2}\right) \longrightarrow\left(u, x^{2}+\sum_{i=1}^{m-2} y_{i}^{2}\right)$ (dobras definidas ou pontos de $\left.S_{o}(f)\right)$;

(ii) $\left(u, x, y_{1}, \ldots, y_{m-2}\right) \longrightarrow\left(u, x^{2}-\sum_{i=1}^{\lambda} y_{i}^{2}+\sum_{i=\lambda+1}^{m-2} y_{i}^{2}\right)$ (dobras indefinidas ou pontos de $S_{1}(f)$ );

(iii) $\left(u, x, y_{1}, \ldots, y_{m-2}\right) \longrightarrow\left(u, \pm u x \pm x^{3}-\sum_{i=1}^{\lambda} y_{i}^{2}+\sum_{i=\lambda+1}^{m-2} y_{i}^{2}\right)$ (cúspides ou pontos de $C(f))$;

$f$ deve ainda satisfazer às seguintes condições globais:

(iv) $f^{-1}(f(p)) \cap S(f)=\{p\}$, sempre que $p \in C(f)$, onde $S(f)$ denota o conjunto singular de $f$;

(v) $\left.f\right|_{S(f)-C(f)}$ é uma imersão com interseções normais.

Denota-se o conjunto das aplicações estáveis de $M$ em $\mathbb{R}^{2}$ por $S\left(M, \mathbb{R}^{2}\right)$.

Observações 3.2.1. (a) Quando $\lambda=0(\lambda>0)$ a singularidade correspondente é dita definida (resp. indefinida).

(b) Uma aplicação $f$ é dita "genérica" se satisfazer as condições anteriores (i), (ii) e (iii).

(c) Uma aplicação $f$ tal que $S(f)=S_{o}(f)$ é dita "genérica especial" (ver capítulo 2, seção 2). 
(d) O conjunto singular $S(f)$ é uma subvariedade fechada 1-dimensional de $M$. Logo, $S(f)$ se expressa como uma união finita e disjunta de curvas difeomorfas a $S^{\mathbf{1}}$. Os subconjuntos $S_{o}(f)$ e $S_{1}(f)$ são abertos em $S(f)$, os quais são círculos ou arcos abertos, em cujas extremidades se encontram os pontos de $C(f)$.

(e) $C(f)$ é um conjunto discreto em $S(f)$, sendo que as cúspides definidas separam arcos de $S_{0}(f)$ de arcos de $S_{1}(f)$, enquanto que as cúspides indefinidas apresentam arcos de $S_{1}(f)$ de ambos os lados. Portanto, o número de cúspides definidas de cada componente conexa de $S(f)$ é sempre par, e estas sempre podem ser canceladas (ver Levine (1965)).

(f) Define-se a fatorização de Stein associada a $f,\left(W_{f}, q_{f}, \bar{f}\right)$, de forma análoga ao que foi definido anteriormente para aplicações genéricas especiais. É interessante observar que, dada uma aplicação contínua $\phi$ entre espaços topológicos, $\phi: X \rightarrow Y$, pode-se definir analogamente a fatorização de Stein $W_{\phi}$ associada a $\phi$. Quando $X$ e $Y$ forem métricos e $X$ compacto pode-se provar que $W_{\phi}$ é métrico e compacto (ver Mata-Lorenzo (1986)).

(g) Se $p \in S(f)$, então $q_{f}^{-1}\left(q_{f}(p)\right) \cap S(f)$ tem no máximo dois pontos singulares. Quando $q_{f}(p)=q_{f}\left(p^{\prime}\right)$, os pontos singulares $p$ e $p^{\prime}$ pertencem necessariamente a $S_{1}(f)$ e denominam-se pontos duplos. Por outro lado, quando $p$ for o único ponto na $q_{f}^{-1}$-imagem de $q(p)$, este denomina-se simples.

Mata-Lorenzo (1986) considera o Problema 3.1.1, obtendo resposta afirmativa quanto à sobrejetividade de $q_{j}$. quando $M$ for uma 3-variedade diferenciável, fechada e orientada. Para tanto, considerando $f: M \rightarrow \mathbb{R}^{2}$ estável e $M$ sob tais hipóteses, Mata-Lorenzo (1986) utiliza as configurações locais admissiveis para o espaço $W_{f}$, 
obtidas por Kushner-Levine-Porto (1984), a saber:

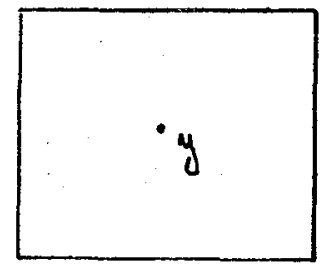

$y=q_{f}(p)$

$q_{f}^{-f}(y) \cap S(f)=\phi$

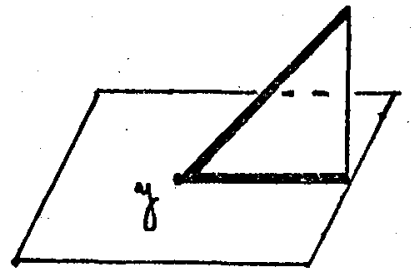

$y=q(p)$

$p \in C(f)$

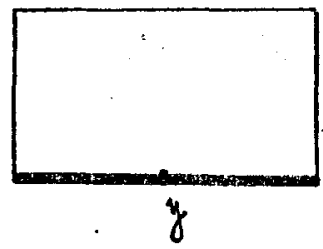

$y=q_{f}(p)$

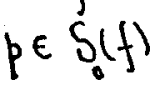

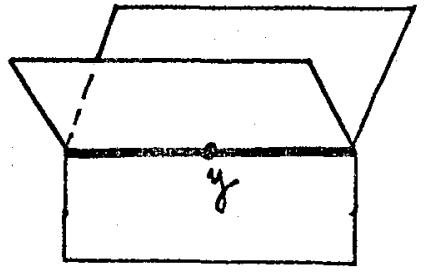

$y=q(p)$ $p \in S_{1}(f)$ é simples

onde as linhas escuras representam as $q_{f}$-imagens de arcos singulares e os números internos representam o número de componentes conexas das $f$-fibras.

Observaçäo 3.2.2 No trabalho de Porto-Furuya (1990), para uma aplicaçào estảvel $f: M \rightarrow \mathbb{R}^{2}$ sendo $M$ uma 4-variedade diferenciável fechada e orientada, encontramos todas as configurações locais admissíveis para $W_{f}$, cujos 10 modelos básicos são dados por:
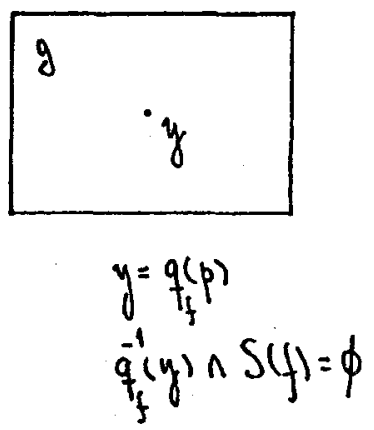

Fig. 3.1

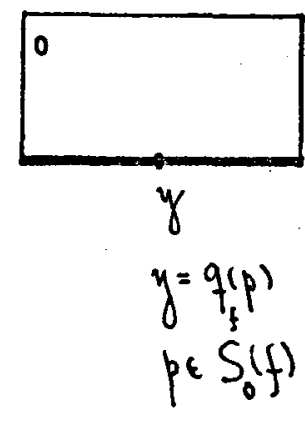

Fig. 3.2 

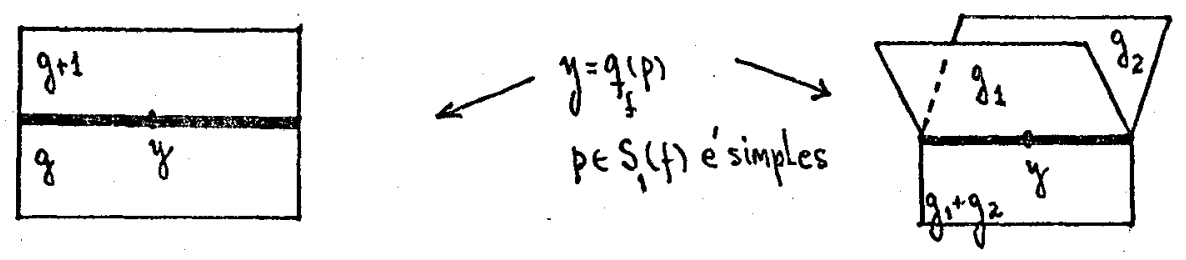

Fig. 3.3

Fig. 3.4

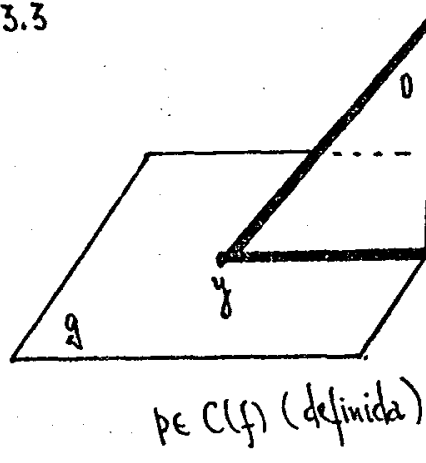

Fig. 3.5

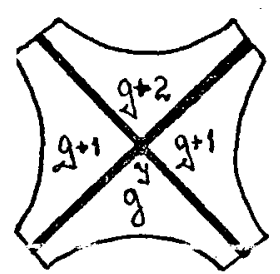

Fig. 3.7

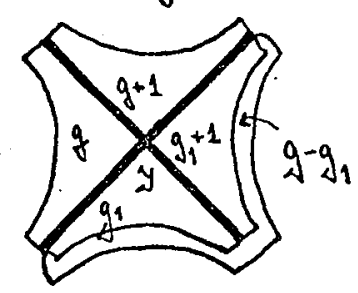

Fig. 3.9<smiles>CC[Te]</smiles>

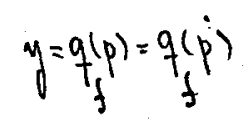<smiles>[Tl]</smiles>
P.P $P^{\prime} \in S_{1}(f)$ SÃO duplos.

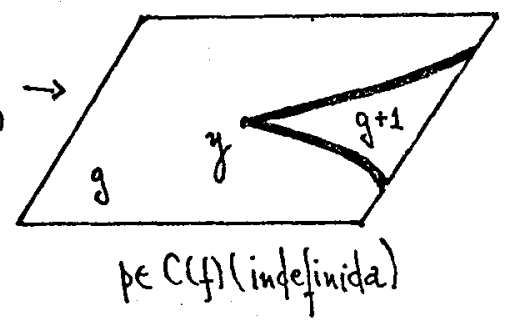

Fig. 3.6

onde as linhas escuras representam as $q_{f}$-imagens de arcos singulares e os símbolos $g_{1}, g_{2}, g_{3}$ e $g$ representam o genus das $q_{f}^{-1}$-imagens de pontos nas respectivas regiões.

O Problema 3.1.2 está relacionado ao Problema 2.1.1, pois é evidente que aplicações genéricas especiais são particulares aplicações com dobras. Observemos 
ainda que quando trabalhamos com aplicações de $M^{3} \rightarrow \mathbb{R}^{2}$, sabendo-se que neste caso a característica de Euler de $M$ é nula, então podemos assumir que tais aplicações não apresentam cúspides (ver teorema (1) em Levine (1965)). Assim, modelos de complexos bidimensionais realizados por 3-variedades diferenciáveis fechadas e orientadas podem ser considerados sem apresentar $q$-imagens de pontos cuspidais. Neste sentido, os trabalhos de Burlet-de Rham (1974) e Porto-Furuya (1990) estão relacionados ao Problema 3.1.2, onde, por exemplo, se considerarmos $W$ difeomorfo ao 2-disco $D^{2}$ ou ao anel $D_{(1)}^{2}$, temos que as únicas variedades $M^{m}$ realizando $W$ são:

$$
\begin{gathered}
W \cong D^{2}-\left\{\begin{array}{l}
M^{3} \cong S^{3}(\text { ver teorema } 2.2 .2) \\
M^{4} \cong S^{4}(\text { ver teorema } 2.2 .3)
\end{array}\right. \\
W \cong D_{(1)}^{2} \longrightarrow\left\{\begin{array}{l}
M^{3} \cong S^{1} \times S^{2}(\text { ver teorema } 2.2 .2) \\
M^{4} \cong S^{1} \times S^{3}(\text { ver teorema } 2.2 .3)
\end{array}\right.
\end{gathered}
$$

onde $\cong$ denota difeomorfismo.

Observação 3.2.3. Na realidade o Problema 3.1.2 é motivado pela seguinte conjectura estabelecida por Porto-Kushner: "Seja $f: M \rightarrow \mathbb{R}^{2}$ uma aplicação estável cujo conjunto singular $S(f)$ apresenta somente dobras simples, sendo $M$ uma 3-variedade diferenciável, fechada e orientada. Suponhamos também que cada vizinhança $N\left(c^{\prime}\right)$ de uma componente $c^{\prime}=q_{f}(c)$ (onde $c$ é uma componente de dobra indefinida) seja trivial $\left(c^{\prime} \times Y\right)$ e nunca o "Y -fibrado torcido não trivial." Então, $W_{f}$ (e consequentemente $M$ ) é descrito através de seis "modelos básicos" de complexos bidimensionais 
listados abaixo:

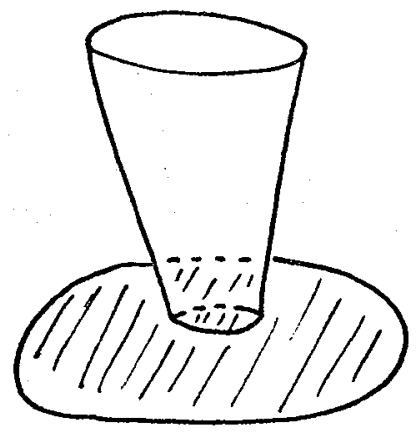

(1)

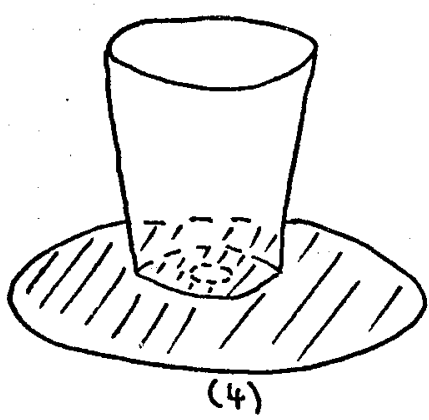

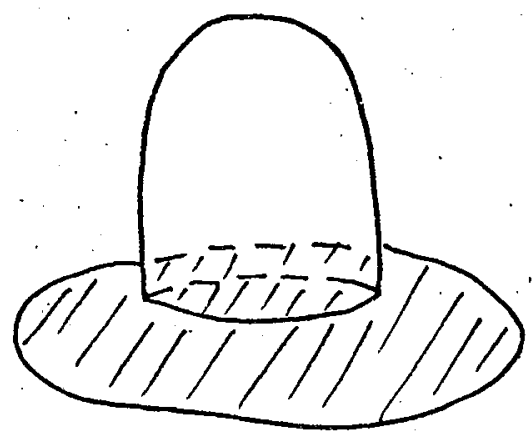

(2)

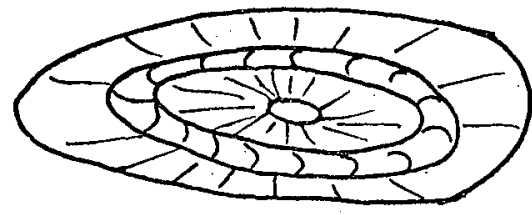

(5)

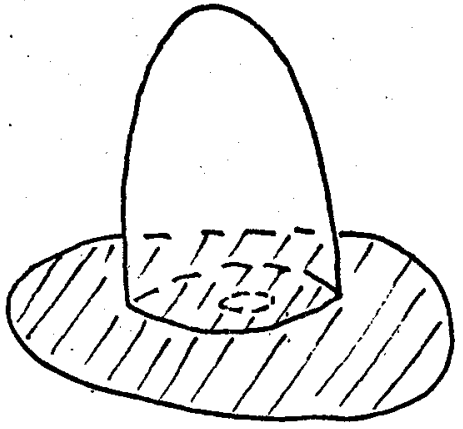

(3)

os quais são realizados unicamente pelas seguintes 3-variedades,
$(1) \rightarrow S^{3}$
$(3) \rightarrow S^{3}$
$(5) \rightarrow S^{1} \times S^{1} \times S^{1}$
(2) $\rightarrow S^{1} \times S^{2}$
$(4) \rightarrow S^{1} \times S^{2}$
(6) $\rightarrow S^{1} \times S^{2} \# S^{1} \times S^{2} . "$

Como poderemos ver adiante tal conjectura éfalsa", haja visto que existem por exemplo modelos básicos admitindo "infinitas" realizações.

Kobayashi (1990) considera o Problema 3.1.2. Trabalhando com uma classe especial $M_{1}$ de 4-variedades diferenciáveis, onde uma 4-variedade diferenciável $M$ pertence a $M_{1}$ se $M$ for fechada , 1-conexa, admitindo uma aplicação

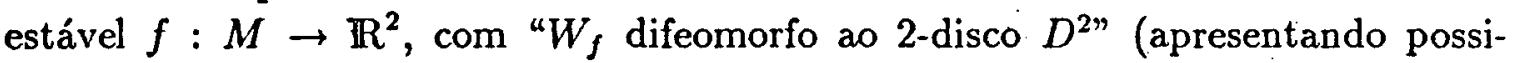
: velmente $q_{f}$-imagens de componentes singulares em seu interior), e, tendo como $q_{f}$-fibras regulares somente esferas ou toros, ele obtem interessantes informações sobre a topologia de $M$ e sobre o conjunto singular de $f$. Mais especificamente, dada $M \in M_{1}$ uma aplicação estável $f: M \rightarrow \mathbb{R}^{2}$ com $W_{f} \cong D^{2}$ é dita "simples" se $f$ 
não apresentar mais do que uma cúspide, $\mathrm{e} g_{f} \mid s(f)$-\{cúspides\} for um mergulho; Então:

Lema 3.2.4 Seja $f: M^{4} \rightarrow \mathbb{R}^{2}$ uma aplicação simples, com $M \in M_{1}$ e seja $\Re$ uma componente conexa de $W_{f}-q_{f}(S(f))$ tal que $\Re \cong D^{2}$. Então, $q_{f}^{-1}(\times) \cong S^{2}, \forall \times \in \Re$.

Observações 3.2.5 (1)Se $M \in M_{1}$, então demonstra-se que sempre existe $f: M \rightarrow$ $\mathbb{R}^{2}$, que é simples. Para tanto. como $M \in M_{1}$ existe uma aplicação estável $\phi: M \rightarrow$ $\mathbb{R}^{2}$ nas condições que definem a classe $M_{1}$. Assim, via deformações locais descritas por homotopias (ver Levine (1965)) podemos modificar $\phi$ localmente, preservando a fatorização como sendo $D^{2}$, e obter $f: M \rightarrow \mathbb{R}^{2}$ estável, nas condições de $M_{1}$, tal que $f$ não tenha cúspides se $\chi(M)$ for par, ou tenha uma única cúspide se $\chi(M)$ for impar. Logo, supondo-se que $\left.q f\right|_{s(f)-\{\text { cusspides\} }}$ tenha pontos duplos, então os genus das fibras regulares ao redor destes pontos säo descritos como abaixo (ver Furuya (1986)):

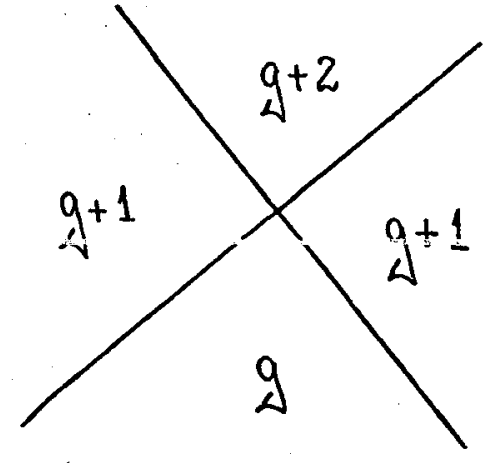

A!

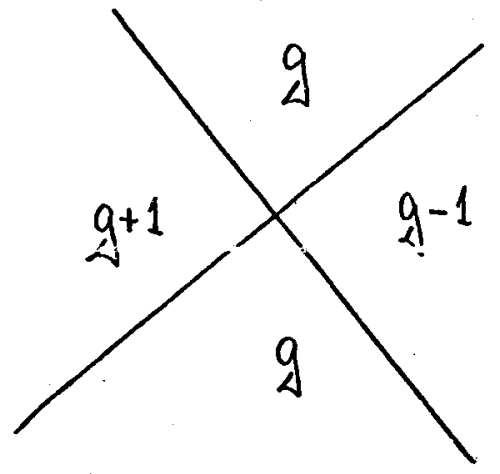

o que contradiz o fato de que o genus é, por hipótese, no máximo 1. Portanto, não podem existir pontos duplos em $S(f)$, ou seja, $\mathfrak{q}_{f} \mid s(f)$-\{cispides\} 
(2) $O$ lema acima nos diz que certos modelos de complexos bidimensionais não são realizáveis por quaisquer $M \in \mathcal{M}_{1}$, por exemplo:

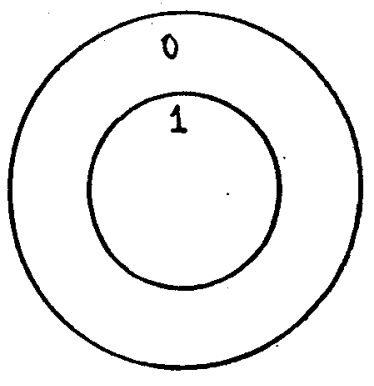

Fig. 3.11

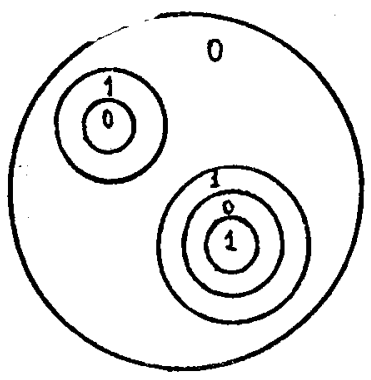

Fig. 3.12

onde os números internos representam o genus das $q_{f}^{-1}$-imagens de pontos sobre tais regiões.

(3) Dada uma componente conexa $W_{o}$ de $W-q(S(f))$ tem-se que $q^{-1}\left(W_{o}\right)$ é um fibrado sobre $W_{o}$ tal que se o fecho de $q^{-1}\left(W_{o}\right)$ contiver pontos de dobra definida, então as $q_{f}^{-1}$-imagens de pontos de $W_{o}$ são esferas bidimensionais (ver corolário 1.11 em Porto-Furuya (1990)). Assim, é obvio que as $q^{-1}$-imagens de dois pontos sobre $W_{o}$ são superfícies de mesmo genus e é por este motivo que nas figuras acima e em outras figuras que se seguem, o inteiro "0" comparece nas regiões adiacentes ao seu exterior. Convem ainda ressaltar que a passagem de uma componente conexa para. outra em $W_{f}-q(S(f))$ produz modificações nos genus relacionados às respectivas componentes e o "controle" sobre o valor do genus é feito através das configuraçôes descritas na observação 3.2.2.

Lema 3.2.6 (Kobayashi (1990)). Seja $f: M^{4} \rightarrow \mathbb{R}^{2}$ uma aplicação simples com $M \in M_{1}$. Seja $J \cong[-1,1]$ um arco transversal a duas componentes de dobras indefinidas, não interceptando a $q_{f}$-imagem da cúspide, e, tal que as extremidades 
de $J$ sejam 2-esferas $S^{2}$ (isto $\left.\dot{c}, q_{f}^{-1}(-1) \cong q_{f}^{-1}(1) \cong S^{2}\right)$ : Então, $q_{f}^{-1}(J) \cong S^{2} \times J$.

Observação 3.2.7. Em outras palavras, o lema 3.2.6 nos diz que os dois pontos singulares determinados por $q_{f}^{-1}(J) \cap S(f)$ são canceláveis (no sentido do "teorema de cancelamento" - ver Milnor (1965)). Assim, podemos deformar $f$ localmente por homotopias e obter uma nova aplicação estável $g: M \rightarrow \mathbb{R}^{2}$ contendo (localmente) a imagem de duas cúspides, como iiustram as Figuras abaixo:

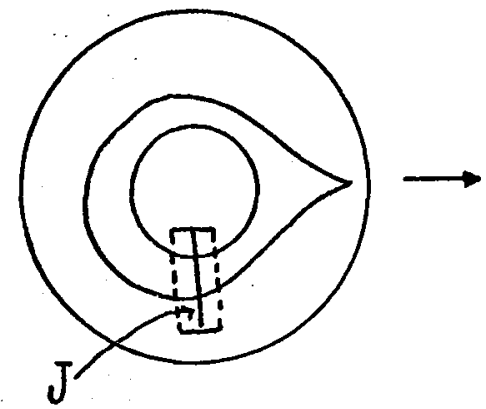

Fig. 3.13

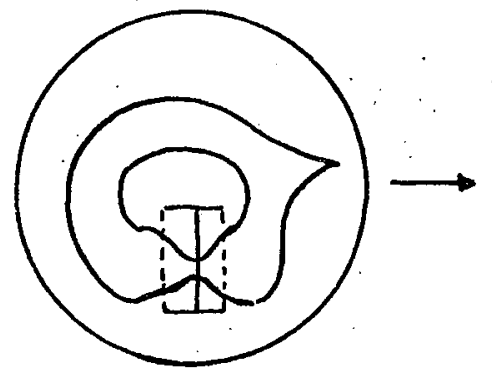

Fig. 3.14

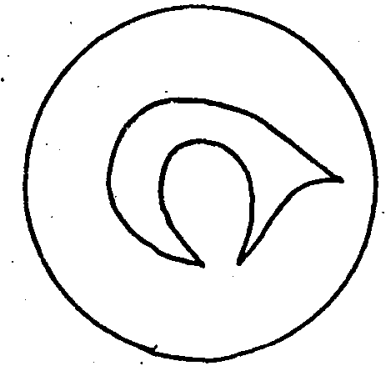

Fig. 3.15

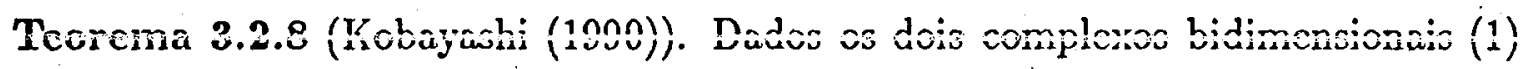
e (2) descritos abaixo, as únicas 4-variedades diferenciáveis, fechadas, 1-conexas e realizando tais complexos são $S^{4}$ e $C P^{2}$ (ou $\overline{C P}^{2} \equiv$ complexo projetivo, com orientação contrária) respectivamente.

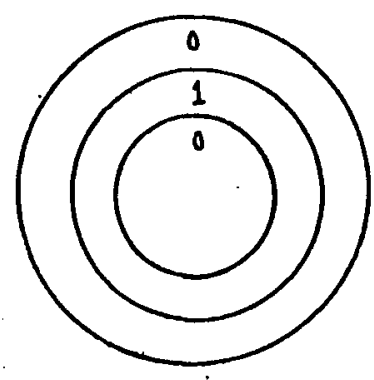

(1)

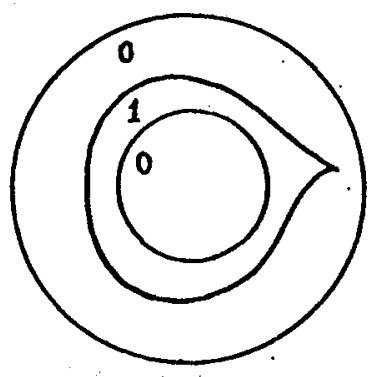

(2) 


\subsection{Resultados Obtidos}

Com respeito ao Problema 3.1.1, utilizando-se as configurações obtidas para $W_{f}$ no caso 4-dimensional (ver 3.2.2), podemos obter resposta afirmativa sobre a sobrejetividade de $q_{f_{.}}$nesta dimensão, como segue.

Lema 3.3.1 Seja $f: M \rightarrow \mathbb{R}^{2}$ uma aplicação estável onde $M$ é uma 4-variedade diferenciável, fechada e orientada. Então, $W_{f}$ é um poliedro bidimensional.

\section{Prova:}

Todos os 10 modelos apresentados em (3.2.2) são naturalmente triangularizáveis. Como $W_{f}$ pode ser coberto por um número finito de tais modelos, pode-se refinar as triangularizações locais subdividindo os complexos simpliciais correspondentes ao longo de algum simplexo 1-dimensional. Desta forma obtém-se uma triangularização global de $W_{f}$.

Teorema 3.3.2 Para $f$ e $M$ nas condições acima tem-se que $q_{f_{*}}: \pi_{1}(M) \rightarrow \pi_{1}\left(W_{f}\right)$ é epimorfismo.

\section{Prova:}

Por (3.3.1), segue-se que $W_{f}$ é um poliedro (complexo simplicial) bidimensional. Consideremos então $(K, \emptyset)$ uma triangulação de $W_{f}$, ou seja, $K$ é um complexo simplicial bidimensional e $\emptyset:|K| \rightarrow W_{f}$ é um homeomorfismo, sendo $|K|=$ $\cup_{s \in K} s$ espaço topológico subjacente a $K$ e $s$ são os simplexos de $K$. Sem perda de generalidade podemos assumir que os 1-simplexos desta triangularização sejam de um dos tipos abaixo:

(a) completamente contidos em $W_{f}-q(S(f))$ com exceção de no máximo 1 vértice;

(b) completamente contidos em arcos de pontos de dobras definidas com exceção de no máximo 1-vértice;

(c) completamente contidos em arcos de pontos de dobras indefinidas com exceção 
de no máximo 1-vértice;

Consideremos uma aplicação contínua e arbitrária $\mu: S^{\mathbf{1}} \rightarrow W_{f}$; se encontrarmos uma aplicação contínua $\beta: S^{1} \rightarrow M$ tal que $q_{f} \circ \beta$ seja homotópica a $\mu, q_{f} \circ \beta \sim \mu$, segue-se o resultado. Na construção de uma tal aplicação contínua $\beta$, considere-se os passos:

$1^{0}$ passo) Constrói-se uma aproximação simplicial $\psi$ de $\mu$ nas condições abaixo.

Seja $(\tilde{K}, \tilde{\emptyset})$ uma triangularização de $S^{1}$ e $(K, \emptyset)$ uma triangulação de $W_{f}$ como descrita acima.

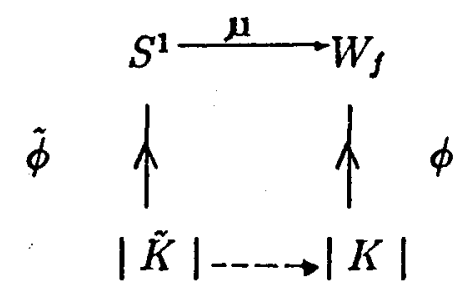

Assim, pode-se considerar $\mu:|\tilde{K}| \rightarrow|K|$. Pelo teorema de aproximação simplicial (ver Rotman (1988)) existe $\psi: S d^{n} \tilde{K} \rightarrow K$ uma aproximação simplicial de $\mu$ para algum inteiro $n \geq 1$, sendo $S \dot{A} A$ subdivisao baricentrica de $A$ onde $S d^{n} A=S d\left(S d^{n-1} A\right)$ e $S d^{\circ} A=A$. Como $\psi$ é aproximação simplicial de $\mu$ temos $|\psi| \sim \mu$. Denotemos por $\mu^{\prime}: S^{1} \rightarrow W_{f}$ o correspondente à $|\psi|$ (a menos de homeomorfismos). Por outro lado, $|\psi|$ pode ser obtida como soma de um número finito de caminhos simples acarretando que $\mu^{\prime}$ é também obtida como soma finita de caminhos simples $a_{i}:[0,1] \rightarrow W_{f}, i=1, \ldots, r$, onde $a_{i}([0,1])$ é 1-simplexo de $W_{f}$ nas condições (a), (b) ou (c) acima.

2ㅇ passo) Com as condições acima, $q_{f}^{-1}\left(a_{i}[0,1]\right)$ é conexo por caminhos.

De fato, $a_{i}[0,1]$ é compacto e conexo em $W_{f}$, e segue-se que $q_{f}^{-1}\left(a_{i}[0,1]\right)$ é métrico, conexo e compacto (ver observação 2.1(e)). Agora, a decomposição de $M$ em fibrados descrita em Furuya (1986) mostra que $q_{f}^{-1}\left(a_{i}[0,1]\right)$ é homeomorfa a um espaço localmente conexo. Portanto, segue-se o desejado.

$\operatorname{Em} q_{f}^{-1}\left(a_{i}[0,1]\right)$ consideremos os pontos $x_{i_{j}} \in q_{f}^{-1}\left(a_{i}(j)\right), j \in\{0,1\}$. Logo, existe 
um caminho $b_{i}:[0,1] \rightarrow q_{j}^{-1}\left(a_{i}[0,1]\right)$ com $b_{i}(0)=x_{i o}$ e $b_{i}(1)=x_{i_{1}}$. Assim, temos que $q_{f} o b_{i}$ é caminho em $W_{f} \operatorname{com} q_{f} o b_{i}(j)=a_{i}(j), j \in\{0,1\}$.

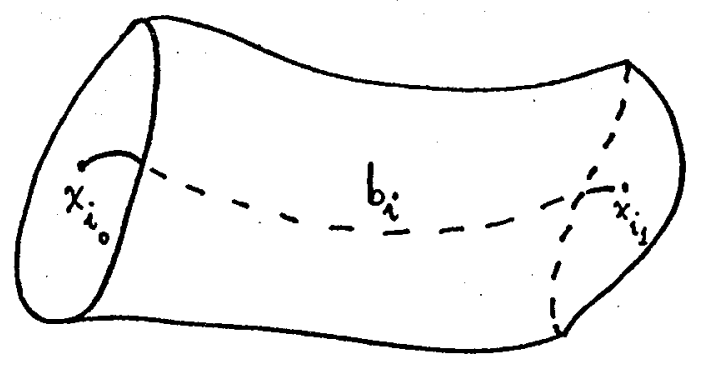

3으 passo) $q_{f} \circ b_{i} \sim a_{i}$ rel. $\{0,1\}$

De fato, $a_{i}$ é homeomorfismo sobre sua imagem; podemos então escrever $q_{f} o b_{i}=$ $a_{i} o c_{i}$, onde $c_{i}:[0,1] \rightarrow[0,1]$ é definido por $c_{i}=a_{i}^{-1} o q_{f} o b_{i} \operatorname{com} c_{i}(0)=0$ e $c_{i}(1)=1$.

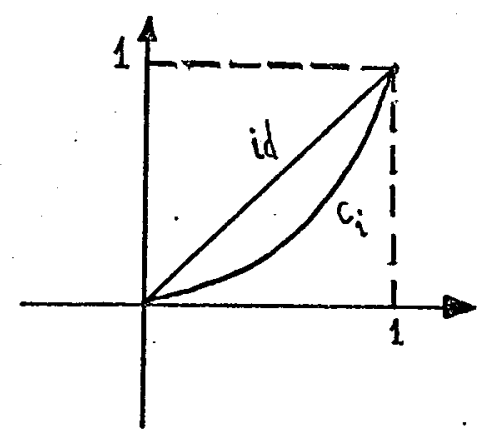

smo $c_{i} \sim$ id rel $\{0,1\}$ segue que $q_{f} o b_{i} \sim a_{i}$ rel $\{0,1\}$.

$4^{0}$ passo) Constrói-se $\beta$ colando-se nas fibras os caminhos $b_{i}$.

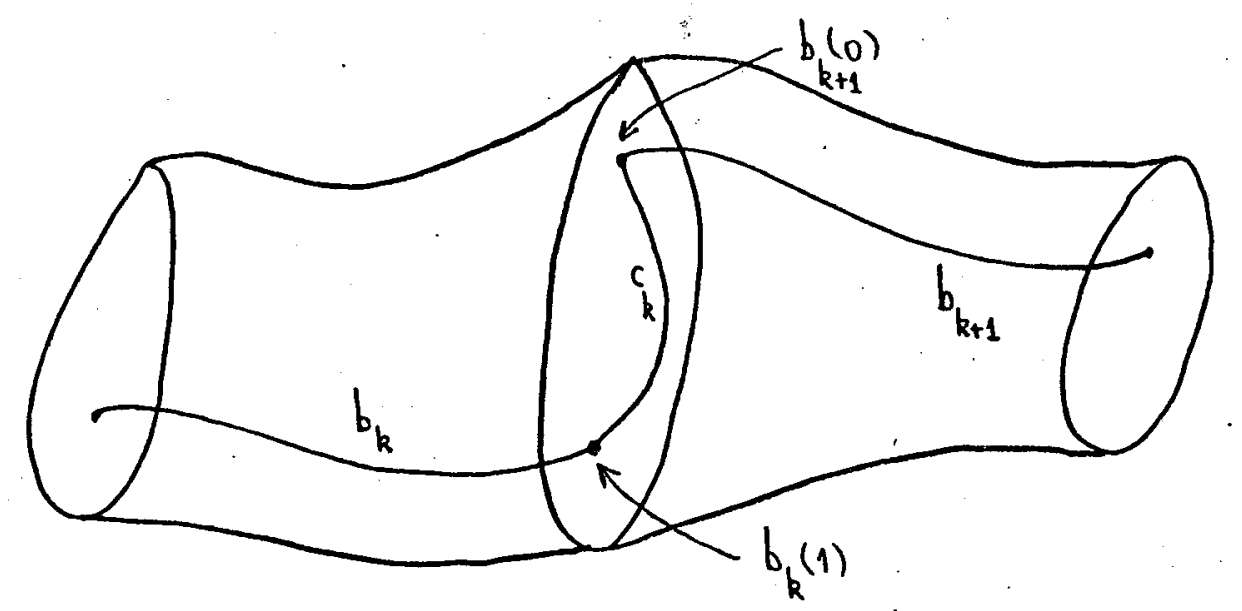

Considerando-se os caminhos $b_{1}, i=1, \ldots, r$, em $M$ com $q_{f} \circ b_{i} \sim a_{i}$ rel $\{0,1\}$, se $b_{i}(1)=b_{i+1}(0)$ tem-se o caminho $b_{i} \cdot b_{i+1}$. Por outro lado, se para algum $k, b_{k}(1) \neq$ 
$b_{k+1}(0)$ (ou $b_{r}(1) \neq b_{1}(0)$ ) então com $b_{k}(1)$ e $b_{k+1}(0)$ estão na mesma q-fibra que por sua vez é conexa por caminhos (ver Furuya (1986)), podemos tomar $c_{k}$ nesta q-fibra de forma a se obter o caminho $b_{k} \cdot c_{k} \cdot b_{k+1}$. Destas justaposiçóes obtem-se $\beta: S^{1} \rightarrow M$ nas condições requeridas.

Observação 3.3.3 Consideremos $f: M^{4} \rightarrow \mathbb{R}^{2}$ como em (3.3.2). Suponhamos que além das condições impostas anteriormente, $M$ seja também 1-conexa e que $W_{f}$ seja uma superfície mergulhada (via $\bar{f}$ ) em $\mathbb{R}^{2}$. Então, $W_{f}$ é difeomorfo a algum $H_{k}=2$-disco com $k$ buracos. Logo, via o epimorfismo $q_{f_{*}}$, segue-se que $W_{f} \cong D^{2}$. Assim, as configurações descritas pelas Figuras 3.4, 3.5, 3.8, 3.9 e 3.10 encontradas na observação 3.2 .2 não podem comparecer em $W_{f}$ nestas condições.

Vamos dividir o nosso estudo relacionado ao Problema 3.1.2 em dois casos:

1 o caso) realizações de complexos bidimensionais por 4-variedades diferenciáveis fechadas;

$2^{\circ}$ caso) realizações de complexos bidimensionais por 3 -variedades diferenciáveis fechadas;

Quanio au primeiro caso, obtemos alguns interessantes resultadios que serão descritos abaixo.

Seja $\phi: S \rightarrow N$ uma imersão com interseções normais, onde $S$ é uma união finita e disjunta de círculos, e $N$ é uma superfície fechada e conexa com $H_{1}\left(N, Z_{2}\right)=0$. Denotemos por $A_{\phi}$ o conjunto dos pontos múltiplos de $\phi$. Como extensão natural dos lemas (2) e (3) obtidos por Biasi-Fuster (1992) temos:

Lema 3.3.4 Se $A_{\phi} \neq \emptyset$, então $\beta_{0}(N-\phi(S)) \geq 2$.\#S+1; onde $\beta_{k}(X)$ denota o k-ésimo número de Betti de $X, \mathrm{e} \# S$ denota o número de componentes conexas de $S$.

\section{Prova:}


Pela sequencia de homologia do par $(N, \phi(S))$ e dualidade, tem-se que $H_{0}(N-$ $\phi(S))=Z_{2} \oplus H_{1}(\phi(S))$. Por outro lado, utilizando-se do lema 3 e notações estabelecidas por Biasi-Fuster (1992), segue-se que $H_{1}(\phi(S))=\oplus_{1}^{\# S} Z_{2} \oplus$ ker $\alpha \geq 2$.\#S. Portanto, $\beta_{0}(N-\phi(S)) \geq 2$.\#S+1.

Em analogia à classe $M_{1}$ definida por Kobayashi (1990), consideremos a classe $\tilde{\mathcal{M}}_{1}$ constituida de 4-variedades diferenciáveis $M$ as quais são fechadas, 1-conexas, com característica de Euler par e admitem uma aplicação estável $f$ : $M \rightarrow \mathbb{R}^{2}$, sendo $W_{f}$ uma superfície mergulhada (via $\bar{f}$ ) em $\mathbb{R}^{2}$, e, $S(f)$ constituido somente de pontos de dobra.

Observações 3.3.5 (1) As variedades $S^{4}, C P^{2}$ e $S^{2} \times S^{2}$ são exemplos de elementos de $\tilde{\mathcal{M}}_{1}$.

(2) Dada uma 4-variedade diferenciável $M$ sendo fechada (orientada)e 1-conexa com $\chi(M)$ par, assumir que $M$ admite uma aplicação estável $f: M \rightarrow \mathbb{R}^{2}$ com $S(f)$ constituido apenas por pontos de dobra não representa restrição, haja visto que, o conjunto das aplicações estáveis de $M$ em $\mathbb{R}^{2}$ é um subçonjunto aberto e denso em $C^{00}\left(M, \mathbb{R}^{2}\right)$ e, como $\chi(M)$ é par, uma aplicação estável $g: M \rightarrow \mathbb{R}^{2}$ através de processos de eliminação de cúspides descritos por Levine (1965), pode ser deformada homotopicamente, sem alterar sua fatorização, de forma a se obter uma aplicação estável de $M$ em $\mathbb{R}^{2}$ com conjunto singular constituido apenas por pontos de dobra.

(3) Seja $f: M \rightarrow \mathbb{R}^{2}$ nas condições acima, como $M$ é 1-conexo e $q_{f_{0}}$ é epimorfismo (ver teorema 3.3.2), segue-se que $W_{f} \cong D^{2}$. Assim, dados $M \in \tilde{\mathcal{M}}_{1}$ e $f: M \rightarrow \mathbb{R}^{2}$ nas condiçôes que definem a classe $\tilde{\mathcal{M}}_{1}$, como $f$ é estável, tem-se que $\left.f\right|_{s(f)}: S(f) \rightarrow$ $\mathbb{R}^{2}$ é uma imersão com interseçôes normais e $S(f)$ é uma união finita e disjunta de círculos. Logo, o lema anterior nos motiva a definição a seguir.

Definição 3.3.6 Sendo $f: M \rightarrow \mathbb{R}^{2}$ nas condições acima, $f$ é dita "trivial" se $\beta_{0}\left(\mathbb{R}^{2}-\left.f\right|_{s(f)}(S(f))\right)=\# S(f)+1$. Em outras palavras, $S(f)$ é constituido apenas por pontos de dobra simples e as únicas configurações locais para $W_{f}$ são as 
descritas pelas Figuras 3.1, 3.2 e $3.3 \mathrm{em}(3.2 .2)$.

Consideremos $M \in \tilde{\mathcal{M}}_{1}$ e seja $f: M \rightarrow \mathbb{R}^{2}$ trivial. Tomemos uma curva $\gamma \in$ $q_{f}\left(S(f)\right.$ ), sendo $\gamma q_{f}$-imagem de uma componente de dobras indefinidas (portanto contida no interior de $W_{f}$ ), e, consideremos $V_{\gamma} \cong J_{\gamma} \times \gamma$, vizinhança tubular de $\gamma$ com $J_{\gamma} \cong[-\epsilon, \epsilon]$ onde $\epsilon$ é um número real positivo e suficientemente pequeno tal que $V_{\gamma} \cap q_{f}(S(f))=\gamma$ e $\{0\} \times \gamma \cong \gamma$. Pode-se dizer (a menos de $\bar{f}: W_{f} \rightarrow \mathbb{R}^{2}$ ) que $\gamma$ divide $\mathbb{R}^{2}$ em uma "região limitada" e outra "ilimitada". Assim, orientamos $J_{\gamma}$ tendo origem $o$ na região limitada e extremidade $e$ na ilimitada. Então, diz-se que $\gamma \in D_{f}$ se a superfície $q_{f}^{-1}(o)$ tiver genus maior que $q_{f}^{-1}(e)$ ( $D_{f} \equiv$ genus decrescente), e, em caso contrário diz-se que $\gamma \in C_{f}\left(C_{f} \equiv\right.$ genus crescente).

Observação 3.3.7 Se $f: M \rightarrow \mathbb{R}^{2}$ é trivial $\left(M \in \tilde{\mathcal{M}}_{1}\right)$ e o número de componentes de $D_{f}$ e $C_{f}$ são iguais, então, por exemplo, as configurações admissiveis para $W_{f}$ são da forma:

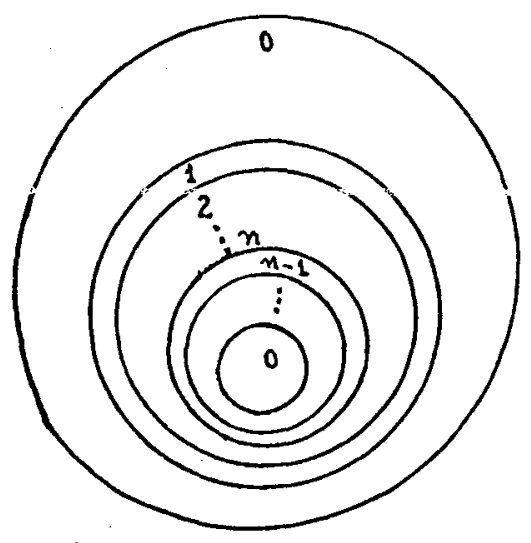

(1)

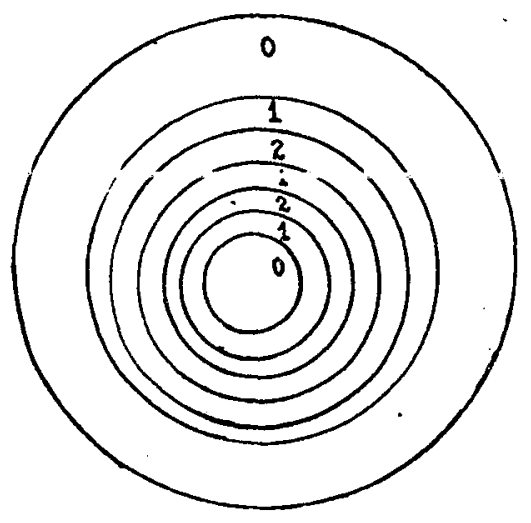

(2)

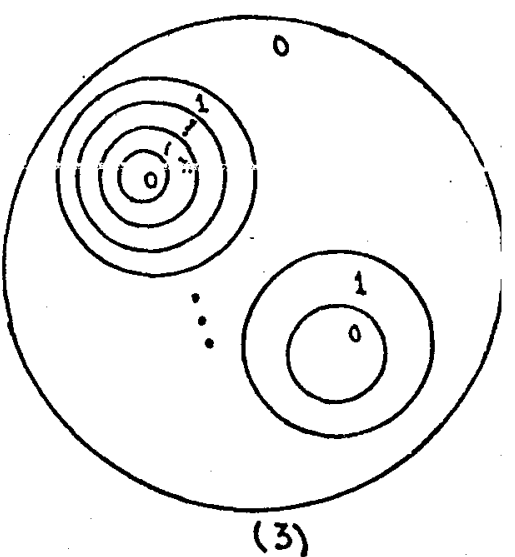

(3)

Enquanto que, por exemplo, os modelos descritos abaixo não são realizáveis por tais 
variedades que admitem aplicações triviais nestas condições:

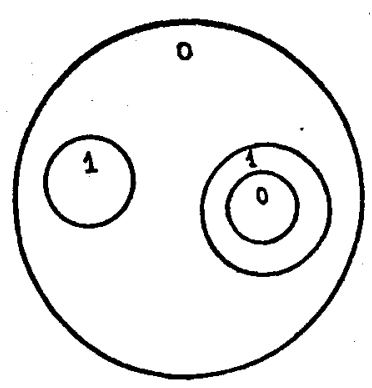

(4)

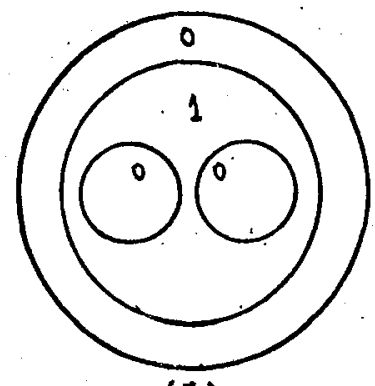

(5)

Teorema 3.3.8 Sejam $M \in \tilde{\mathcal{M}}_{1}$ e $f: M \rightarrow \mathbb{R}^{2}$ trivial com \# $D_{f}=\# C_{f}$. Sendo $q_{f}^{-1}(x)$ uma superfície de genus menor ou igual a $1, \forall x \in W_{f}-q_{f}^{-1}(S(f))$, então $M$ é difeomorfa a $S^{4}$, onde $\# D_{f}\left(\# C_{f}\right)$ representa o número de componentes de $D_{f}\left(C_{f}\right)$.

\section{Prova:}

Seja $\mathcal{R}$ componente conexa de $W_{f}-q_{f}(S(f))$ com $\mathcal{R} \cong D^{2}$. Logo, pelo lema 3.2.4, tem-se que $q_{f}^{-1}(x) \cong S^{2}, \forall x \in \mathcal{R}$. Assim, podemos dizer que a configuração $\left({ }^{*}\right)$ descrita abaixo é "normal" em $W_{f}$ :

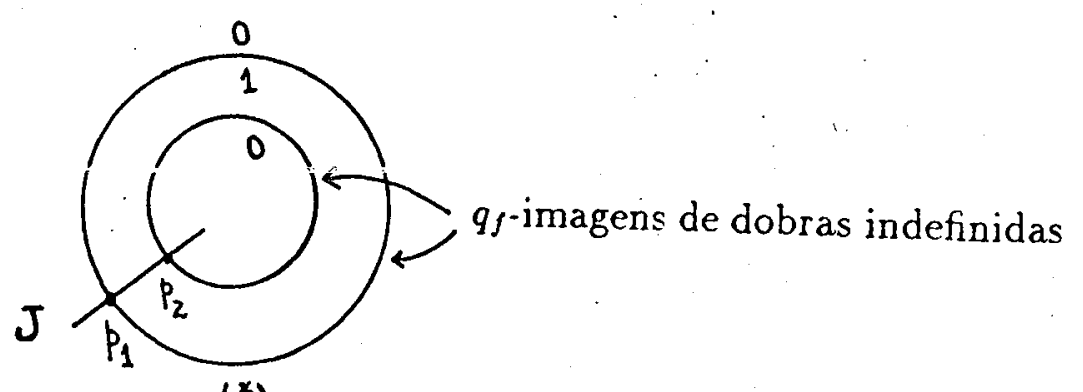

$(*)$

Logo, tomando-se $J \cong[-1,1]$ transversal às $q_{f}$-imagens de dobras indefinidas em $\left(^{*}\right)$, tem-se que $q_{f}^{-1}(J) \cong S^{2} \times J$ (ver lema 3.2.6). Então, os pontos críticos $q_{f}^{-1}\left(p_{i}\right)$ sâo canceláveis, de forma que podemos deformar localmente $f$, via o teorema de cancelamento (ver Milnor (1965)), obtendo uma nova aplicação estável com duas 
cúspides canceláveis:

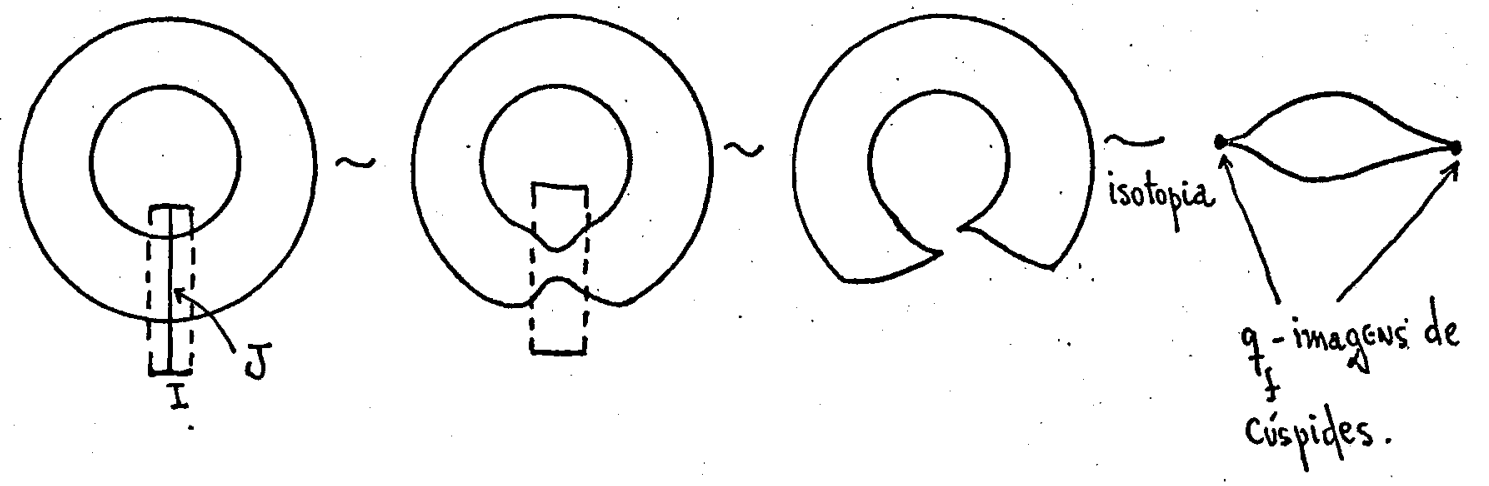

Agora, podemos deformar localmente $f$, por uma homotopia $\pi$-estável (ver Furuya (1986)), de tal forma que as transições das fatorizações ao longo desta homotopia são descritas por:

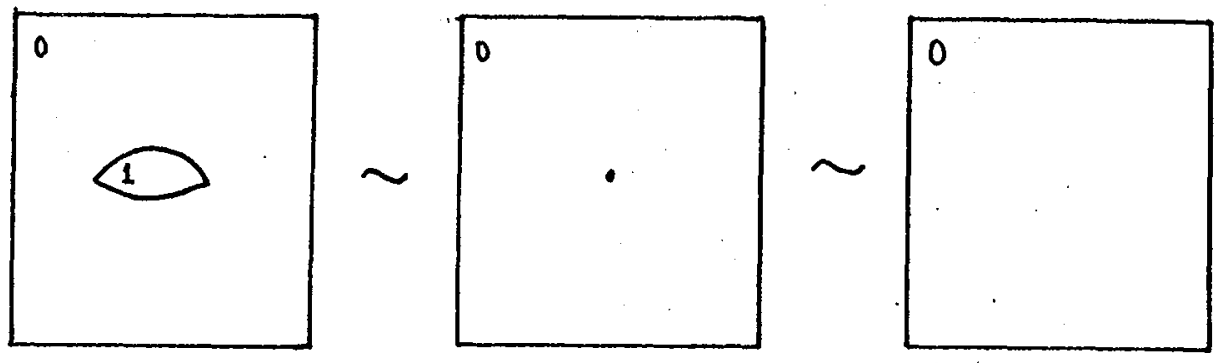

Portanto, nrocedendo sucessivas vezes como acima, obtemos uma aplicação $g: M \rightarrow \mathbb{R}^{2}$ tal que $W_{g} \cong D^{2}$ e $g$ é genérica especial. Logo, pelo teorema 2.2.3 (1) tem-se o resultado.

Observemos que o modelo (5) em 3.3.7 somente não satisfaz a condição \# $D_{f}=$ $\# C_{f}$. Tal modelo foi alvo de estudos de Kobayashi (1990), o qual utilizando argumentos encontrados em Levine (1966), obteve que variedades $M \in M_{1}$ que realizam (5), com $f: M \rightarrow \mathbb{R}^{2}$ simples, tem o segundo número de Betti, $b_{2}(M)$, igual a 2. Assim, associamos a tais variedades uma forma bilinear simétrica $\omega$.definida como no capítulo $2, \omega: H^{2}(M, Z) \otimes H^{2}(M, Z) \rightarrow Z, \omega(x, y)=<x \cup y,[M]>$. Denotando 
o indice de $M$ por $\sigma(M)$, segue que:

Teorema 3.3.9 Seja $f: M^{4} \rightarrow \mathbb{R}^{2}$ realizando o modelo (5), com $\sigma(M)=0$. Então, $M$ é homotopicamente equivalente a:

$$
\begin{cases}S^{2} \times S^{2}, & \text { se } \omega \text { for par } \\ C P^{2} \# \overline{C P}^{2}, & \text { se } \omega \text { for impar. }\end{cases}
$$

\section{Prova:}

Por hipótese $M$ é 1-conexa e como $b_{2}(M)=2$, por dualidade, segue que $\chi(M)=$ 4. Assim, como $\sigma(M)=0$, então $M$ é uma equivalência de homotopia com o espaço total de um $S^{2}$-fibrado sobre $S^{2}$ (ver teorema 5 em Hillman (1991)). Por outro lado, existem exatamente dois $S^{2}$-fibrados sobre $S^{2}$ (tais fibrados são classificados por $\pi_{1}(S O(3))$ o qual é um grupo de ordem 2, ver Steenrod (1951)), com espaços totais dados por $S^{2} \times S^{2}$ ou $S^{2} \tilde{x} S^{2} \cong C P^{2} \# \overline{C P}^{2}$.

Observação 3.3.10 Sejam $f_{i}: M_{i} \rightarrow \mathbb{R}^{2}(\mathrm{i}=1,2)$ aplicaçôes estáveis, sendo $M_{i} 4$ variedades diferenciáveis fechadas, orientadas e conexas. Assim, podemos construir uma aplicação estável $f_{1} \# f_{2}: M_{1} \# M_{2} \rightarrow \mathbb{R}^{2}$ como segue: tomemos um ponto $a_{i} \in \partial W_{f_{i}}$ e um arco mergulhado $\gamma_{i}$ em $W_{f_{i}}$ o qual conecta dois pontos de $\partial W_{f_{i}}$ e é transversal a $\partial W_{f_{i}}$ tal que $\gamma_{i}$ contorna $a_{i}$ como abaixo:
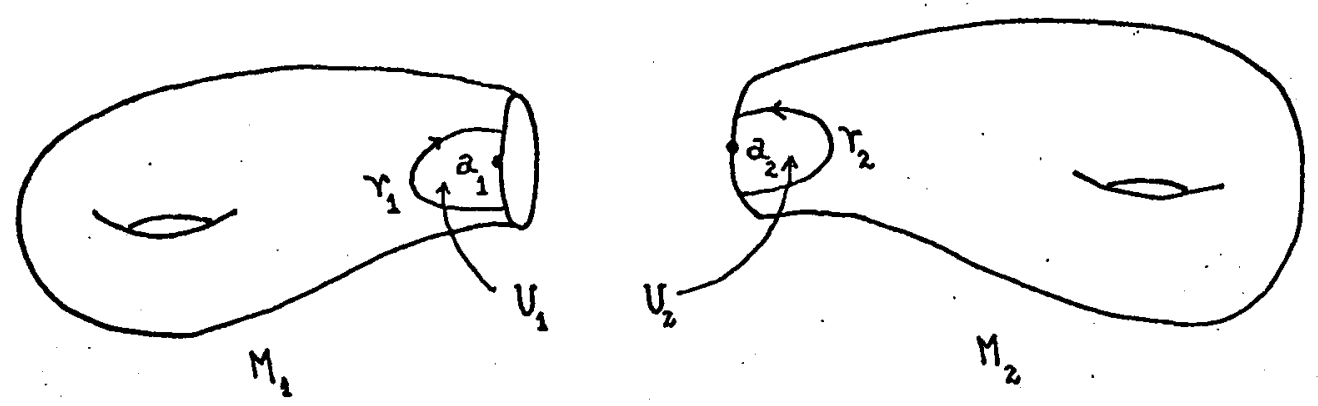

Seja $U_{i}$ a região limitada por $\gamma_{i}$, em $W_{f_{i}}$, contendo $a_{i}$. Então, por Levine (1966), tem-se que $q_{f_{i}}^{-1}\left(U_{i}\right) \cong D^{4}$ e $q_{f_{i}}^{-1}\left(\gamma_{i}\right)=\partial q_{f_{i}}^{-1}\left(U_{i}\right)$. Por Levine (1985), as duas funções 
$\left.q_{f_{i}}\right|_{q_{i}^{-1}\left(\gamma_{i}\right)}: S^{3} \rightarrow \gamma_{i}$ são funções de Morse sobre $S^{3}$ e são equivalentes. Assim, sejam $\phi$ e $\psi$ difeomorfismos comutando o diagrama abaixo,

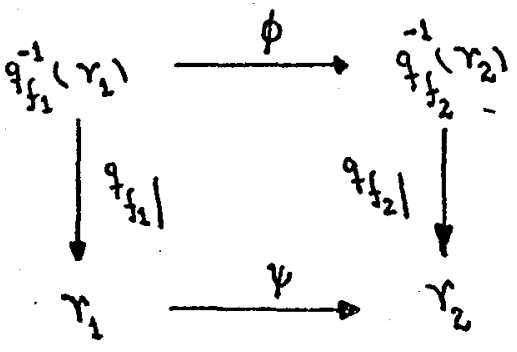

Observe-se que podemos tomar $\phi$ invertendo orientação, pois caso contrário, tomamos $k: q_{f_{2}}^{-1}\left(\lambda_{2}\right) \rightarrow q_{f_{2}}^{-1}\left(\lambda_{2}\right)$ um difeomorfismo invertendo orientação o qual satisfaz $\left.q_{f_{2}}\right|_{\tilde{q}_{f_{2}}^{-1}\left(\gamma_{2}\right)}=\left.q_{f_{2}}\right|_{q_{f_{2}}\left(\gamma_{2}\right)} ^{-1}\left(\lambda_{2}\right.$ e inverte a orientação de cada superfície de nível de

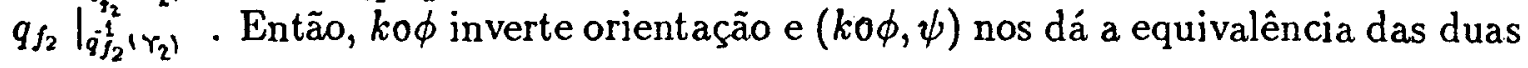
funçôes de Morse.

Consideremos $M_{i}^{\prime}=\overline{M_{i}-q_{f_{i}}^{-1}\left(U_{i}\right)}$ e $W_{f_{i}}^{\prime}=\overline{W_{f_{i}}-U_{i}}$. Quando $\psi$ inverte orientação, podemos colar as triades $\left(M_{i}^{\prime},\left.q_{f_{i}}\right|_{M_{i}^{\prime}}, W_{f_{i}}^{\prime}\right)$ por $\phi$ e $\psi$ e obter $\left(M_{1} \# M_{2}, \tilde{q}, W_{f_{1}} \# W_{f_{2}}\right)$, onde $\tilde{q}$ é uma $C^{\circ}$-aplicação satisfazendo $\tilde{q}=q_{f_{i}}$ sobre $M_{i}^{\prime}$. Através de uma pequena perturbação, transformamos $\tilde{q}$ em uma aplicação estável tal que $\tilde{q}$ e $q_{f_{i}}$ são equivalentes (à esquerda) sobre $M_{i}^{\prime}$. Agora, modificamos a imersão $\bar{f}_{2}$ por uma equi valência (à esquerda) de modo a $f_{2}\left(M_{2}^{\prime}\right)$ colar-se com $f_{1}\left(M_{1}^{\prime}\right)$. Então, $\bar{f}_{1}$ e $\bar{f}_{2}$ podem também ser coladas e nós obtemos uma imersão $h: W_{f_{1}} \# W_{f_{2}} \rightarrow \mathbb{R}^{2}$. Portando, temos uma aplicação estável hoq $: M_{1} \# M_{2} \rightarrow \mathbb{R}^{2}$, a qual denotamos por $f_{1} \# f_{2}$.

Por outro lado, se $\psi$ preservar orientação, tomemos $l: W_{f_{2}}^{\prime} \rightarrow W_{f_{2}}^{\prime}$ um difeomorfismo invertendo orientação. Então, colamos as triades $\left(M_{1}^{\prime}, q_{\left.f_{1}\right|_{M_{1}^{\prime}}}, W_{f_{1}}^{\prime}\right)$ e $\left(M_{2}^{\prime}, q_{\left.f_{2}\right|_{M_{2}^{\prime}}}, W_{f_{2}}^{\prime}\right)$ por $\psi$ e $\left.l\right|_{\gamma_{2}}{ }^{\circ} \psi$. Analogamente ao caso acima, modificando a imersão $\bar{f}_{2} o^{-1}$ obtemos uma aplicação estável $f_{1} \# f_{2}: M_{1} \# M_{2} \rightarrow \mathbb{R}^{2}$.

Portanto, obtemos uma aplicação estável $f_{1} \# f_{2}: M_{1} \# M_{2} \rightarrow \mathbb{R}^{2}$ determinada por $f_{1}$ e $f_{2}$, a qual é única a menos de equivalência e satisfaz as condições:

(1) $f_{1} \# f_{2}$ é equivalente a $f_{i}$ sobre $M_{i}^{\prime}, \mathrm{j}=1,2$

(2) $W_{f_{1} \# f_{2}}=W_{f_{1}} \# W_{f_{2}}$

(3) $q_{f_{1} \# f_{2}}$ é equivalnte a $q_{f_{i}}$ sobre $M_{i}^{\prime}, \mathrm{i}=1,2$ 
Obstrvação 3.3.11 Kobayashi (1990) estabelece que $M \in \mathcal{M}_{1}$ realizando o modelo (a) descrito abaixo é difeomorfo a $C P^{2}$. Então, se considerarmos no modelo (5) a seguinte situação:

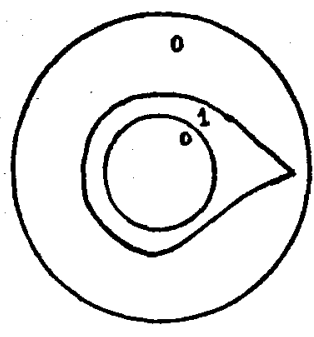

(a)

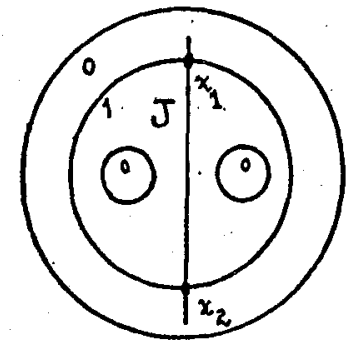

(5)

seja $J \cong[-1,1]$ transversal à componente de dobra indefinida como acima (na realidade é transversal a sua $q$-imagem). Se $q_{f}^{-1}\left(x_{i}\right)=p_{i}(\mathrm{i}=1,2)$ forem "pontos críticos canceláveis", então pode-se deformar (5) e descrevê-lo como abaixo:

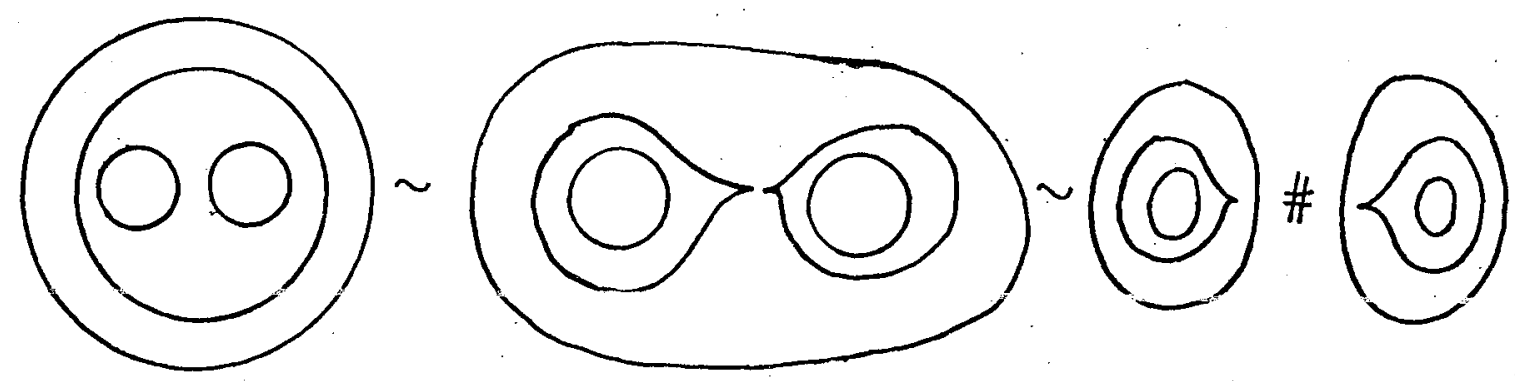

Logo, pela observação acima e o teorema 3.2.8, tem-se que $M$ realizando (5) sob tais condições é difeomorfo a $C P^{2} \# C P^{2}$. Pode-se descrever (via funções de Morse) $q_{f}^{-1}(J)$ como a colagem de dois toros sólidos menos dois 3-discos abertos contidos cada um deles no interior de um respectivo toro sólido. Assim, $q_{f}^{-1}(J) \cong \hat{L}(p, q)$ (espaço de lens removendo-se 2 discos $D^{3}$ ). Logo, $p_{1}$ e $p_{2}$ são canceláveis se $p=1$ pois $\hat{L}(1, q) \cong \hat{S}^{3} \cong S^{2} \times J$. É então natural que se ponha a seguinte questão, na qual estamos trabalhando: "que condições adicionais devem ser impostas à $M$ para - se garantir que $\mathrm{p}=1$ ?

Vamos agora considerar os nossos estudos relacionados ao ( $2^{\circ}$ caso), ou seja, realizações de complexos por 3-variedades diferenciáveis e fechadas. 
$\mathrm{Na}$ observação 3.2.3 são apresentados seis "modelos básicos de complexos", e se tem uma conjectura sobre suas realizações. As observações abaixo mostram quais são as realizaçôes de alguns destes modelos, de onde se observa as impossibilidades da utilização de tais modelos no problema proposto por Porto-Kushner.

Observações 3.3.12 (1) Dado o complexo bidimensional (*) descrito abaixo; então se $M$ for uma 3-variedade diferenciável realizando $\left({ }^{*}\right)$ tem-se que $M$ é difeomorfa a uma das seguintes variedades: $S^{1} \times S^{2} ; S^{3}$ ou o espaço de lens $S^{3} / Z_{n}$, onde $Z_{n}$ atua livremente sobre $S^{3}=\left\{z \in C^{2} /\|z\|=1\right\}$ por $m .\left(z_{1}, z_{2}\right)=\left(\xi^{m} \cdot z_{1}, \xi^{m} \cdot z_{2}\right)$, sendo $\xi$ uma n-ésima raiz primitiva de 1 .

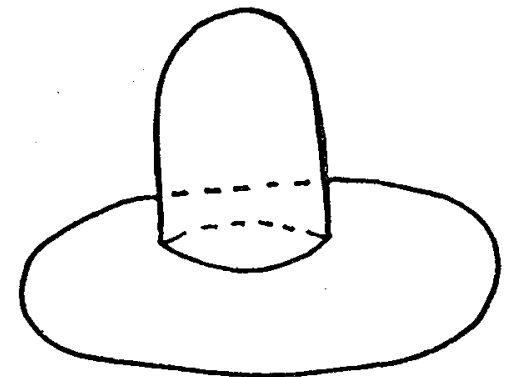

$(*)$

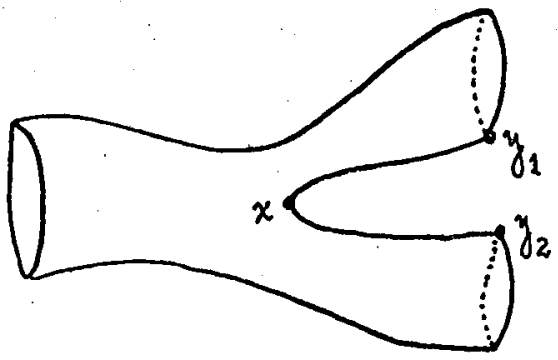

$(* *)$

De fato: na configuração (**) acima $x$ represent.a um ponto de sela e $y_{1}$ e $y_{2}$ são dois "pontos marcados". Em uma vizinhança da componente de $\left({ }^{*}\right)$ abaixo em negrito temos duas familias de "pontos marcados":

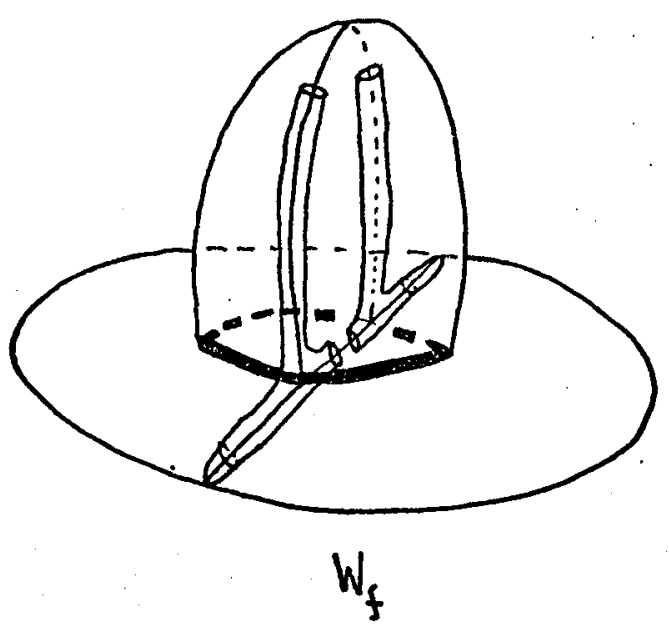




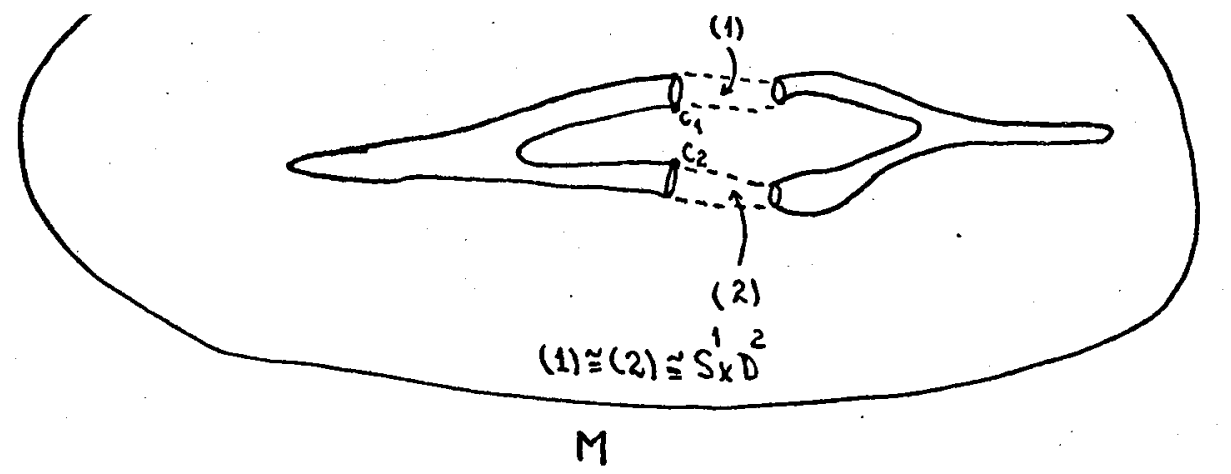

Em (1), os pontos marcados formam um círculo $c_{1}$ em $S^{1} \times S^{1}=\partial\left(S^{1} \times D^{2}\right)$, e, em (2) tais pontos formam um círculo $c_{2}$ também contido no $\partial\left(S^{\prime} \times D^{2}\right)$. Sejam $n_{i}(\mathrm{i}=1,2)$ o "número de vezes que $c_{i}$ se enrola $\mathrm{em} S^{1} \times D^{2}$ ". Então, tais números determinam completamente $M$, sendo que: se $n_{1}=n_{2}$, então $M=S^{\prime} \times S^{2}$; se $n_{1}-n_{2}= \pm 1$, então $M=S^{3}$ e se $n_{1}-n_{2}= \pm n$, então $M=S^{3} / z_{n}$ (ver Rolfsen (1976)).

(2) Consideremos agora o complexo bidimensional $\left({ }^{* *}\right)$ descrito a seguir,

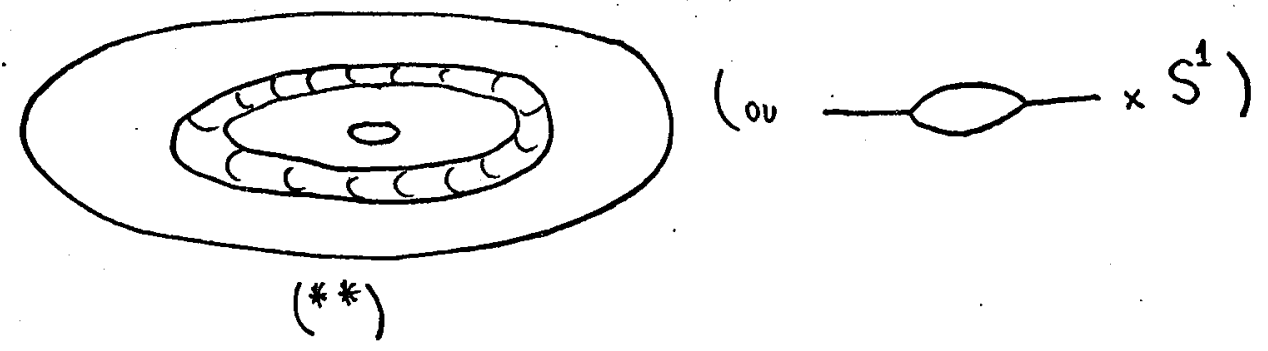

Logo, as 3-variedades realizando $\left({ }^{* *}\right)$ são classificadas por um par de inteiros (n, $\mathrm{m}$ ) os quais contam o número de vezes que as "marcas" sobre os círculos $c_{1}$ e $c_{2}$ 
enrolam-se ao redor do "topo" e da "base" respectivamente como na figura abaixo:
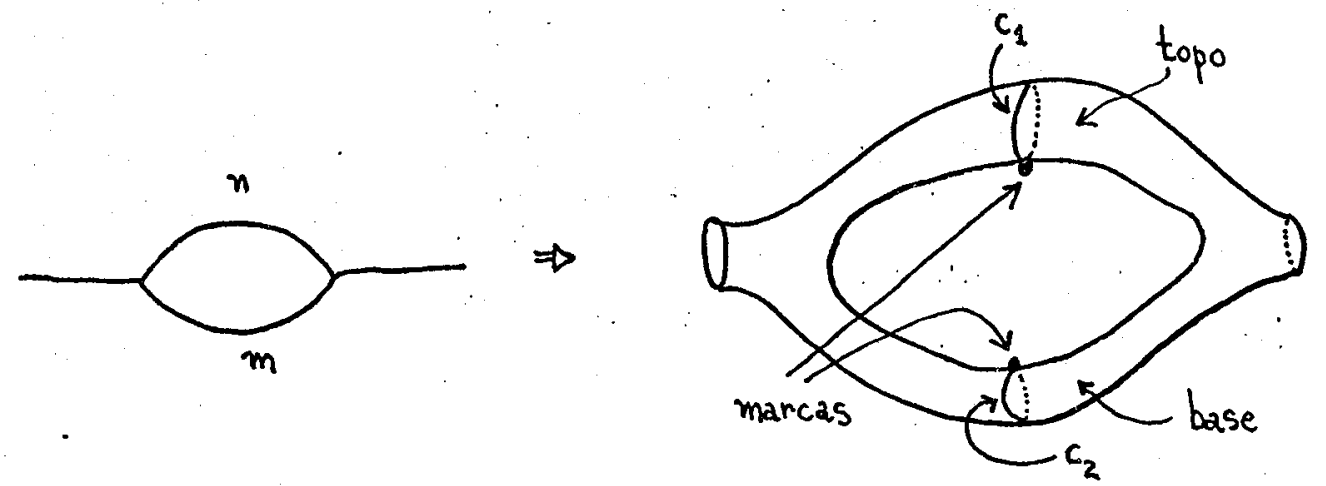

Assim, as 3-variedades realizando (**) são da forma $S^{1} \times S^{1} \times I / f$, onde $I \cong[0,1]$ e $f: S^{1} \times S^{1} \times\{0\} \rightarrow S^{1} \times S^{1} \times\{1\}$ é o "entrelaçamento de Dehn" $f=\left(\begin{array}{cc}1 & m-n \\ 0 & 1\end{array}\right)$.

Consideremos $M$ uma 3-variedade diferenciável, fechada, conexa e orientada. Então, $M$ tem uma "decomposição de Heegaard" $M=M_{1} \cup_{\psi} M_{2}$, onde $M_{1}$ e $M_{2}$ são dois "handlebodies" sólidos 3-dimensionais de mesmo genus com $\partial M_{1}=\partial M_{2}=$ $S$ sendo uma superfície conexa, e a aplicação $\psi: S \rightarrow S$ é um "difeomorfismo de colagem". Nestas condições, pode-se obter um difeomorfismo $\phi: S \rightarrow S$ tal que $M_{1} \cup_{\phi} M_{2}=S^{3}$. Observemos, ainda que qualquer difeomorfismo $\phi$ de uma superficie $S$ é isotópico a composição de "entrelaçamento de Dehn" (Dehn twists), e que $M_{1} U_{\phi} M_{2}$ depende somente da classe de isotópia de $\phi$. Para as demonstrações dos resultados descritos acima ver Dubrovin (1990). Assim, podemos estabelecer o resultado:

Teorema 3.3.13 Seja $M$ uma 3-variedade diferenciável, fechadia, conexa e orientada. Então, existem aplicações genéricas $f: M \rightarrow \mathbb{R}^{2}$ e $g: S^{3} \rightarrow \mathbb{R}^{2}$, e um homeomorfismo $h: W_{f} \rightarrow W_{g}$ comutando o diagrama abaixo:

Prova:

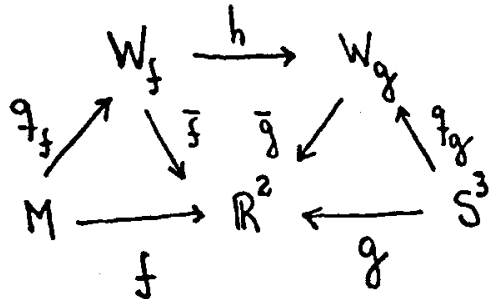


Tomemos uma decomposiçâo de Heegaard de $M, M=M_{1} \cup_{\psi} M_{2}$, nas condiçôes acima.

Seja $F: M \rightarrow \mathbb{R}$ uma função de Morse tal que 0 é valor regular de $F, M_{1}=$ $F^{-1}(-\infty, 0]$ e $M_{2}=F^{-1}[0, \infty)$. Consideremos $\epsilon$ um número real positivo suficientemente pequeno para que $F$ não tenha valores críticos em $(-\epsilon, \epsilon)$. Assim, escolhemos números $a_{i}, \mathrm{i}=1, \ldots, \mathrm{n}$ tais que $0<a_{1} \ldots<a_{n}<\epsilon$ são valores regulares de $F$. Logo, nas subvariedades $S_{i}=F^{-1}\left(a_{i}\right)$ contidas em $M$ tomemos curvas fechadas $c_{i}$ para cada $i$. Nestas condições, existe uma aplicação genérica $f: M \rightarrow \mathbb{R}^{2}, f=\left(f_{1}, f_{2}\right)$, tal que $f_{1}=F,\left(a_{i}, 0\right)$ é valor regular de $f$ e $c_{i}$ é uma componente de $f^{-1}\left(a_{i}, 0\right)$.

Por outro lado, escolhemos um difeomorfismo $\phi: S \rightarrow S\left(S=\partial M_{1}=\partial M_{2}\right)$ tal que $M_{1} \cup_{\phi} M_{2}=S^{3}$, e $\phi$ (a menos de isotópia) é expresso pela composição $\phi=\phi_{n} o \ldots o \phi_{1}$, onde $\phi_{i}$ é um entrelaçamento de Dehn sobre a curva $c_{i}$ respectivamente. Observe que $f_{2} \circ \phi_{i}=f_{2}$ sobre cada $S_{i}$. Logo, $M_{\phi}=M_{1} \cup_{\phi} M_{2}=$ $\tilde{M}_{0} \cup_{\phi_{1}} \tilde{M}_{1} \cup \ldots \cup_{\phi_{n}} \tilde{M}_{n}$ onde $\tilde{M}_{o}=F^{-1}\left(-\infty, a_{1}\right], \tilde{M}_{i}=F^{-1}\left[a_{i}, a_{i+1}\right](0<i<n) \mathrm{e}$ $\tilde{M}_{n}=F^{-1}\left[a_{n}, \infty\right)$. Nestas condições, existe uma aplicação genérica $g: M_{\phi} \rightarrow \mathbb{R}^{2}$ (tome $\left.g\right|_{\dot{M}_{i}}=\left.f\right|_{\dot{M}_{i}}$ ) e um homeomorfismo $h: W_{f} \rightarrow W_{g}$.

Observação 3.3.14 Sabe-se que a característica de Euler de uma 3-variedade diferenciável, fechada, conexa e orientada é nula. Assim, utilizando-se o teorema (1) em Levine (1965), podemos deformar as aplicações $f$ e $g$ dadas acima, de forma a obter novas aplicações $\tilde{f}$ e $\tilde{g}$ nas condições de (3.3.13) e que não apresentam cúspides (suas únicas singularidades são dobras). Pana a construucăo de f axima ver Apéndice.

Consideremos agora dois complexos bidimensionais descritos pelas Figuras 3.16 


\section{e 3.17 abaixo:}
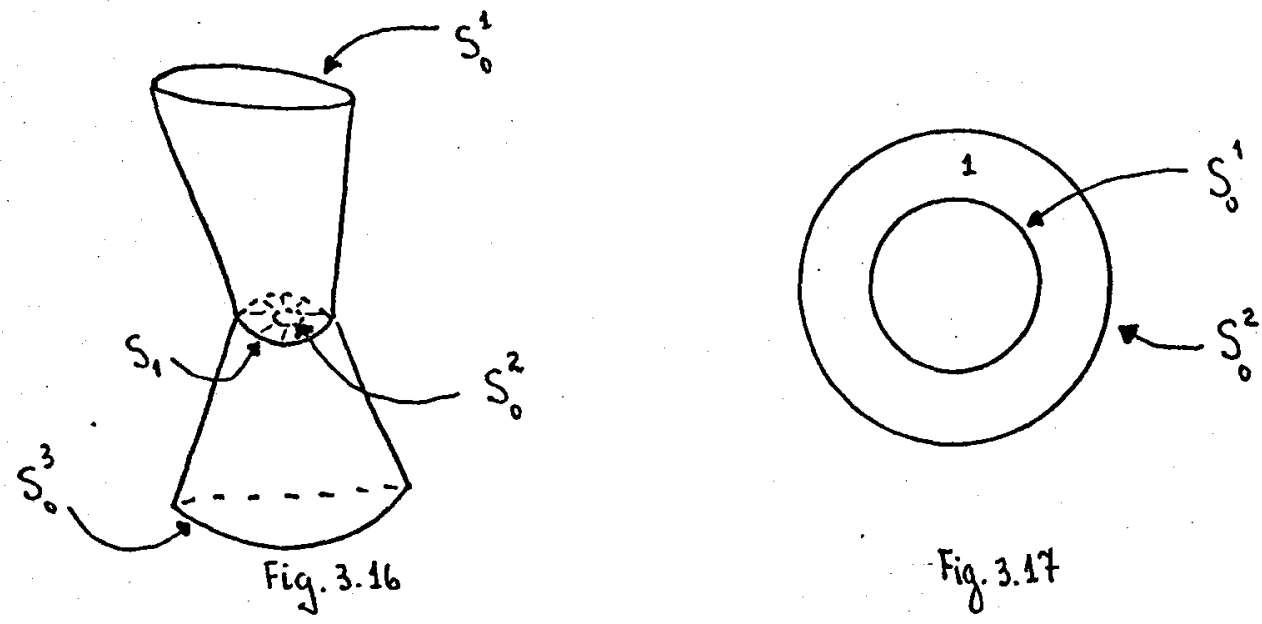

onde $S_{o}^{i}$ correspondem a dobras definidas, $S_{1}$ corresponde a dobras indefinidas e os números internos correspondem ao número de componentes de $f$-fibras.

Pelo teorema de Realização (ver Mata-Lorenzo (1986)), existem realizações $\left(M_{1}, f_{1}\right)$ e $\left(M_{2}, f_{2}\right)$ das Figuras 3.16 e 3.17 respectivamente, com $M_{i}$ sendo uma 3-variedade diferenciável, fechada e orientada e $f_{i}: M_{i} \rightarrow \mathbb{R}^{2}$ estável. Assim, pelo teorema 2.2.2, segue que $M_{2} \cong S^{1} \times S^{2}$, sendo $f_{2}$ genérica especial. Façamos um estudo sobre a realização do modelo descrito na Figura 3.16. Tomemos neste modelo um ponto arbitrário de dobra indefinida, $x_{0}$. Logo, utilizando-se de resultados obtidos por Levine (1985) temos a existência (local) de:

- um mergulho de $I \times J \cong(-1,1) \times[-1,1]$ em $\mathbb{R}^{2}$ tal que $f_{1}\left(x_{0}\right)$ corresponde a $(0,0)$;

- uma aplicação diferenciável $g: I \times T \rightarrow J,\left.\operatorname{com} g\right|_{\{u\} \times T:\{u\} \times T \rightarrow J}$ sendo uma função de Morse, onde $T$ é a componente conexa de $f_{1}^{-1}(\{0\}$ $\times J)$ contendo $x_{0}$;

- um mergulho topológico de $W_{1 \times g}$ sobre sua imagem em $W_{f_{1}}$. 
Assim, localmente, $\left(M, f, W_{f}\right)$ corresponde à configuração descrita a seguir:

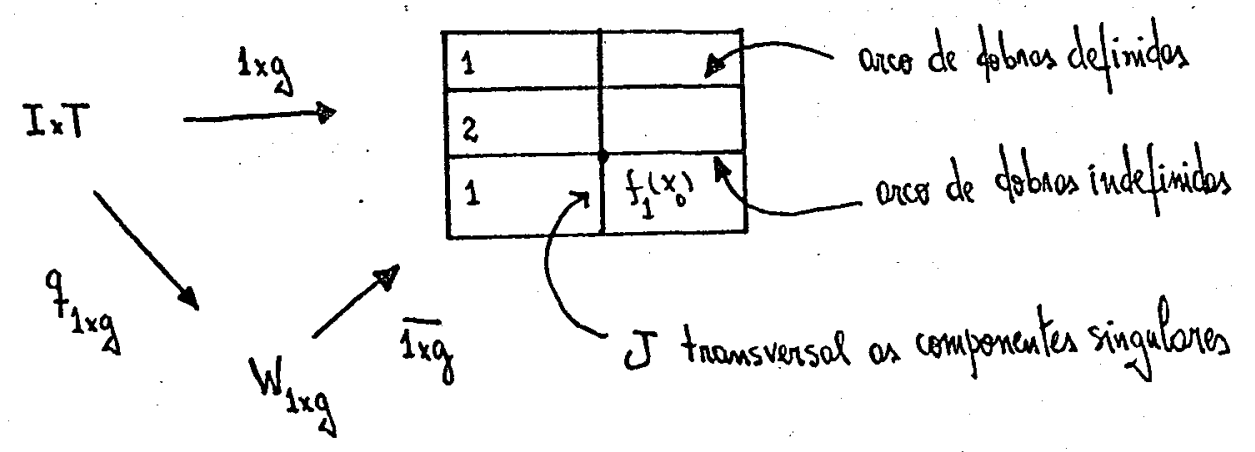

onde $T \cong D_{(1)}^{2}\left(=S^{2} \times I\right)$ é um 2-disco com um buraco correspondendo a Hirsch (1976)).

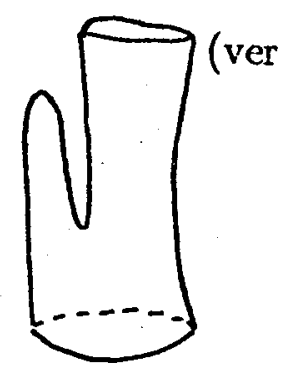

Demonstremos que nas condiçôes acima, $W_{1 \times g}=I \times Y$. Para tanto, construiremos um difeomorfismo $\emptyset: I \times T \rightarrow I \times T$, com $\emptyset(t, x)=\left(t, \emptyset_{t}(x)\right)$, tal que modulo este difeomorfismo $1 \times g$ é da forma $1 \times h$, onde $h$ é a função altura

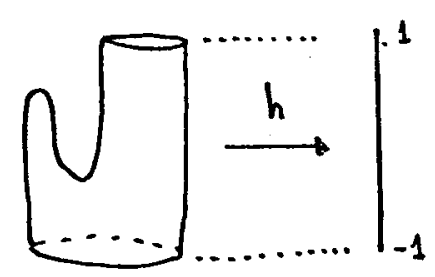

Afirmação 1. Seja $\phi: I \times D^{2} \rightarrow I \times[0,1]$ uma aplicação diferenciável com $\phi(t, x)=\left(t, \phi_{t}(x)\right)$. Suponhamos que para qualquer $t \in I, \phi_{t}$ seja uma função de Morse com justamente uma singularidade de indice zero no interior de $D^{2}$, e, cujo valor crítico é zero, e sendo ainda $\phi_{t}\left(\partial D^{2}\right)=1$. Então, existe difeomorfismo $A: I \times D^{2} \rightarrow I \times D^{2}$ tal que $\phi_{0} A=1 \times h_{1}$, onde $h_{1}: D^{2} \rightarrow \mathbb{R}$ é definido por 
$h_{1}(x, y)=x^{2}+y^{2}$

Afirmação 2. Seja $\psi: I \times D_{(2)}^{2} \rightarrow I \times J$ uma aplicação diferenciável com $\psi(t, x)=$ $\left(t, \psi_{t}(x)\right)$. Suponhamos que para qualquer $t \in I, \psi_{t}$ seja uma função de Morse com justamente uma singularidade de índice 1 no interior de $D_{(2)}^{2}$, e, tal que duas componentes do bordo de $D_{(2)}^{2}$ tenham 1 como $\psi_{t}$-imagem e a outra componente tenha -1 como $\psi_{t}$-imagem. Então, existe difeomorfismo $B: I \times D_{(2)}^{2} \rightarrow I \times D_{(2)}^{2}$ tal que $\psi o B \doteq 1 \times h_{2}$ onde $h_{2}$ é a função altura

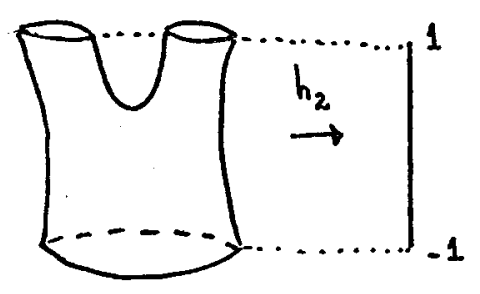

Observação 3.3.15 As demonstrações das afirmações acima podem ser encontradas em Mata-Lorenzo (1986), são bastante técnicas e elaboradas, envolvendo importantes resultados, tais como Lema de Morse Parametrizado (ver Igusa (1986)) e Teorema de Extensão de Isotopia (ver Hirsch (1976)). Assim, a menos dos difeomorfismos $A$ e $B$ acima, tem-se que $W_{\psi}=I \times \downarrow$ e $W_{\psi}=I \times Y$.

Lema 3.3.16 Seja $f: I \times D_{(1)}^{2} \rightarrow I \times J$ uma aplicação diferenciável com $f(t, x)=$ $\left(t, f_{t}(x)\right)$. Suponhamos que para qualquer $t \in I, f_{t}$ seja uma função de Morse com somente duas singularidades, sendo uma de índice 1 e outra de índice dois, ambas no interior de $D_{(1)}^{2}$, e tais que seus valores críticos são 0 e $\frac{1}{2}$ respectivamente. Se as $f_{t}$-imagens de duas componentes do $\partial D_{(1)}^{2}$ forem $1 \mathrm{e}-1$ respectivamente, então existe um difeomorfismo $F: I \times D_{(1)}^{2} \rightarrow I \times D_{(1)}^{2}$ tal que $f o F=1 \times h$, onde $h$ é a 
função altura

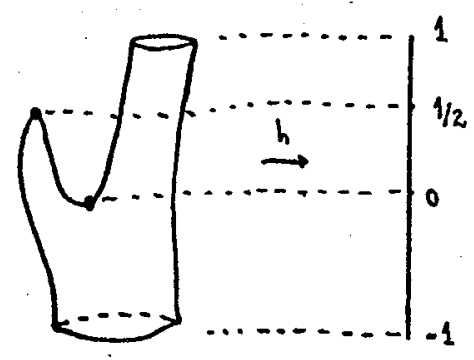

\section{Prova:}

Tomemos $t$ positivo suficientemente pequeno, e, próximo do máximo (local) de $f_{t}$ retiramos um 2-disco $\tilde{D}$. Assim, $\tilde{D}_{(1)} \cong D_{(2)}^{2} \cup_{h} \tilde{D}$, onde $h$ é difeomorfismo entre o bordo de $\tilde{D}$ e a correspondente componente do bordo de $D_{(2)}^{2}$. Qualquer difeomorfismo isotópico a $h$ induz a mesma estrutura diferenciável sobre o "espaço colado" (ver Hirsch (1976)). Agora, como $h \in D i f f_{+}\left(S^{1}\right)\left(D_{(1)}^{2}\right.$ é orientado) e sendo que $\pi_{0}\left(D\right.$ if $\left.f_{+}\left(S^{1}\right)\right)=0$, tem-se que a construção de $D_{(1)}^{2}$ via "colagem" é independente de $h$.

Assim, decompomos $f_{t}=\phi_{t} \cup_{h} \psi_{t}$ onde $\phi_{t}: \tilde{D} \rightarrow[0,1]$ e $\psi_{t}: D_{(2)}^{2} \rightarrow J$. Logo, podemos decompor $f$ por, $f=(1 \times \phi) \cup_{h}(1 \times \psi) \operatorname{com} 1 \times \phi: I \times \tilde{D} \rightarrow I \times[0,1] \mathrm{e}$ $1 \times \psi: I \times D_{(2)}^{2} \rightarrow I \times J$ nas condições das afirmações acima.

Portanto, encontramos $F$ dado por $F_{t}=A_{t} \cup_{h} B_{t}$ nas condições requeridas.

Assim, a configuração local de $\left(M_{1}, f_{1}, W_{f_{4}}\right)$ corresponde a um $D_{(1)}^{2}$-fibrado sobre $I$, onde pode-se construir uma homotopia regular realizando (localmente) as deformações
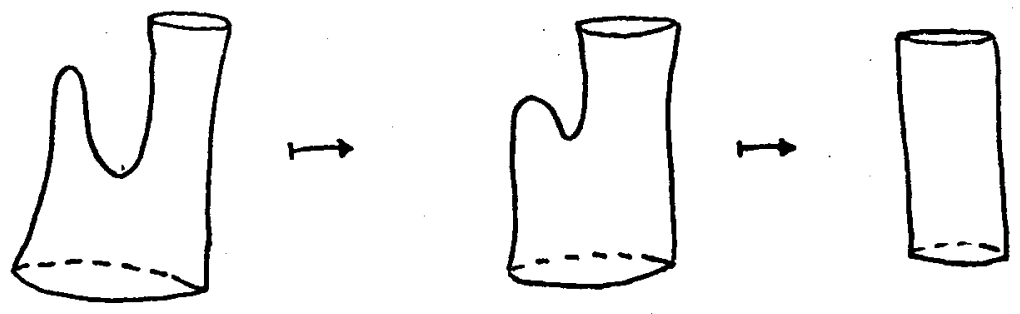

(ver Igusa (1986))

Desta forma, modificando $1 \times h$ (correspondente a $1 \times g$ a menos de difeomorfismo) 
em $q^{-1}(I \times Y)$, construimos uma nova aplicação $\tilde{f}_{\mathcal{L}}$ que é estável tendo duas cúspides na correspon dente configuração local, descrita geometricamente abaixo:
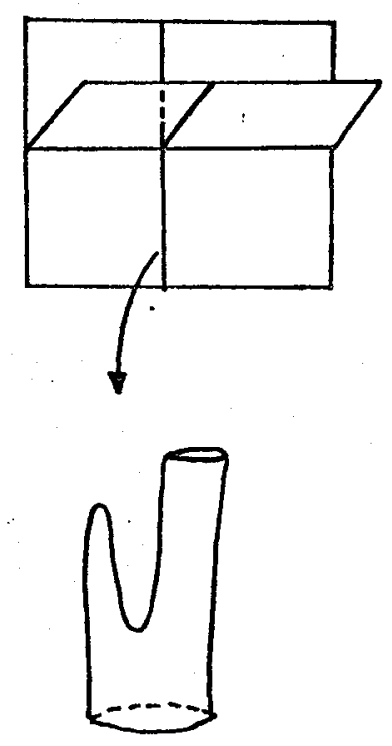
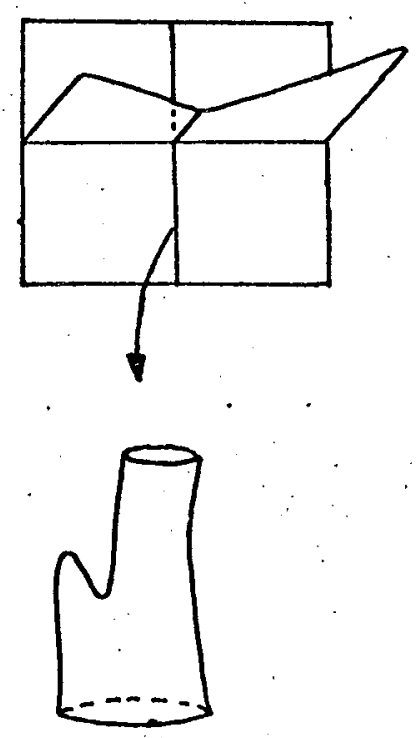
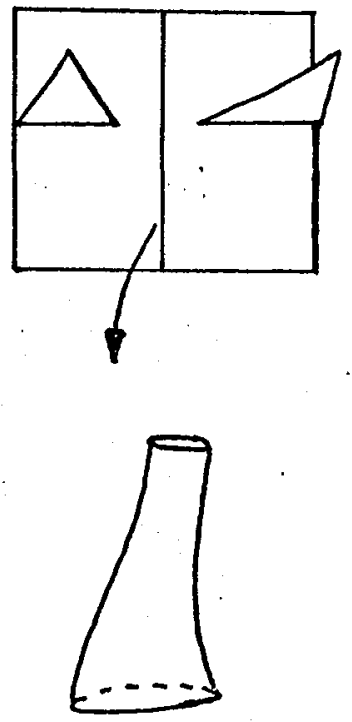

Portanto, $W_{f_{1}}$, pode ser descrito por,
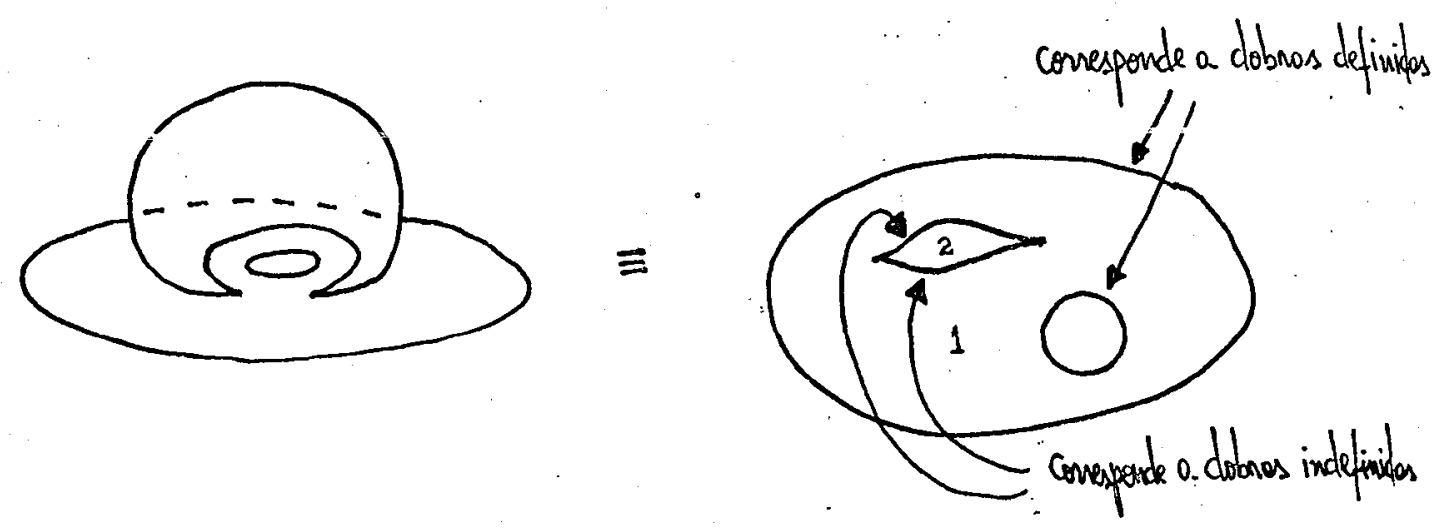

que a menos de homotopia se reduz a segue que:

(

Teorema 3.3.17 0 modelo descrito na Figura 3.16 se realiza unicamente por 
$S^{\prime} \times S^{2}$

Observação 3.3.18 Seja $f: M^{3} \rightarrow \mathbb{R}^{2}$ estável com $W_{f}$ descrito a seguir. Então, utilizando-se os mesmos argumentos acima expostos podemos concluir que $M$ é difeomorfa a $S^{3}$.

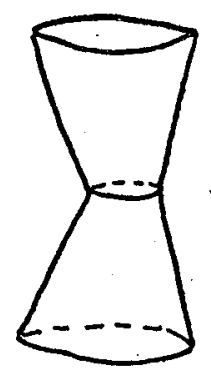

$W_{f}$ 


\section{Bibliografia}

Ballesteros J.J.N. (1991) - Sobre la función de bitangencia asociada a uma curva genérica em $\mathbb{R}^{3}$, Tesis Doctoral, Universitat de Valencia.

Ballesteros J.J.N. and Fuster, M.C.R. (1992) - Separation properties of continuous maps in condimension-1 and geometrical applications (to appear in Topology and its Applications).

Biasi C. and Fuster, M.C.R. (1992) - A converse of the Jordan - Brouwer theorem (to appear in Illinois Journal of Math.).

Burlet O. and de Rham, G. (1974) - Sur certaines applications génériques d'une variété closé a 3-dimensions dans le plan, Enseign. Math. 20:275-292.

Cerf J. (1968) - Sur les difféomorphisues de la sphère de dimension trois $\left(\Gamma_{4}=0\right)$, Lecture Notes in Math., 53, Springer-Verlag.

Cerf J. (1970) - La stratification naturelle des espaces de fonctions différentiables réelle et le théorème de la pseudo-isotopie, Publ. math. de l'Inst. des Hantes Etudes Sci., 39.

Donaldson D. (1983) - An application of gauge theory to the topology of 4manifolds, J. Diff. Geom., 18:269-316.

Dubrovin B.; Fomenko, A. and Novikov, S. (1990) - Modern geometry-methods in applic., Part-III - Introd. to homology theory, GTM 124, Springer-Verlag.

Èliăsberg J.M. (1970) - On singularities of folding type, Math. USSR Izv.4: 1119113.

Feighn M.E. (1988) - Separation properties of codimension-1 immersions, Topology, 27:319-321.

Freedman M. (1982) - The topology of 4-manifolds, J. Diff. Geom., 17:357-453.

Freed D. and Uhlenbeck, K (1984) - Instantons and four-manifolds, Math. Sci. Res. Institute 1. 
Furuya Y.K.S. (1986) - Sobre aplicações genéricas $M^{4} \rightarrow \mathbb{R}^{2}$, Tese de Doutorado, ICMSC-USP.

Fukuda T. (1987) - Topology of folds, cusps and Morin singularities, In: A Fete of Topology, Tokyo, Academic Press: 331-353.

Golubitsky M. and Guillemin, V. (1973) - Stable mapping and their singularities, GTM 14, Springer-Verlag.

Hatcher. A.E. (1983) - A r roof of the Smale conjecture $\operatorname{Diff}\left(S^{3}\right) \simeq S O(4)$, Annals of Math., 117:553-607.

Herbert R.J. (1981) - Multiple points of immersed manifolds, Memoirs of the AMS 34 (250).

Hirsch M. (1976) - Differential topology, GTM 33, Springer-Verlag.

- Hiratuka J.T. (1992) - Aplicaçōes Genéricas Especiais de uma Variedade Fechada de Dimensão $n+1$ no $R^{n}$ - DiSSERTACĂo de MESTRADO - IME.

Hillman J.A. (1991) - On 4-manifolds homotopy equivalent to surface bundles over surfaces, Topology and its Applications 40:275-286.

Igusa K.(1986) - Parametrized Morse theory, In: 5@ Encontro Brasileiro de Topologia, ICMSC-USP:93-10\%.

Kauffman L.H. (1979) - Planar surface immesions, Illinois Jour. of Math., 23(4):648665 .

Kervaire M. and Milnor, J. (1963) - Groups of homolopy spheres, Annols of Math., $77(3): 504-537$.

Kobayashi M. (1990) - Studying manifolds over simple discriminants, Sûrikaisekikenkŷsho Kôkŷroku, 725:81-89 (Math. Reviews 91i:58018).

Kushner L. (1982) - On mappings into $\mathbb{R}^{21}$, Bol. SBM, 13(1):45-54.

Kushner L.; Levine, H. and Porto, P. (1984) - Mapping 3-manifolds into the plane, Bol. Soc. Mexicana, 29(1):11-34.

Levine H. (1965) - Elimination of cusps, Topology, 3:263-296. (1966) - Mappings of manifolds into the plane, Amer. J. of Math., 88(2):357365. 
(1985) - Classifying immersions into $\mathbb{R}^{4}$ over stable maps of 3-manifolds into $\mathbb{R}^{2}$, Lecture Notes in Math. 1157, Springer-Verlag.

Levine J. (1970) - An algebraic classification of some knots of codimension two, Comment. Math. Helv., 45:185-198.

Mata-Lorenzo L.E. (1986) - The Stein factorization of stable maps and stable arcs of maps from 3-manifolds into the plane, Doctor Thesis, Brandeis University. (1986) - Stable mappings from 3-manifolds into the plane and immersed polyhedra, In: 5 Encontro Brasileiro de Topologia, ICMSC-USP:103-109. (1989) - Polyhedrons and pi-stable homotopies from 3-manifolds into the plane, Nova Série Bol. SBM, 20(1):61-85.

Milnor J. (1958) - On simply - connected 4-manifolds. In: Symposium Inter. de Topologia Algebrica, México, 122-128. (1965) - Lectures on h-codordism theorem, Princeton Univ. Press.

Milnor J. and Stasheff, J. (1974) - Characteristic classes, Ann. of Math. Stud.76, Princeton Univ. Press.

Porto P.F.S. (1983) - Informações topológicas sobre o domínio de certas aplicações estáveis, Tese de Livre-Docência, ICMSC-USP.

Porto P.F.S. and Furuya, Y.K.S. (1990) - On special generic maps from a closed manifold into the plane, Topology and its Aplications, 35:41-52.

Rolfsen D. (1976) - Knots and links, Publish or Perisch Inc, Berkeley.

Rotman J. (1988) - An introduction to algebraic topology, GTM 119, SpringerVerlag.

Saeki O. (1992) - Topology of special generic maps (to appear in Topology and its Applications).

Smale S. (1956) - Generalized Poincaré's conjecture in dimensions greather than 4, Annals of Math., 64:399-405. 
(1959) - Diffeomorphisms of the 2-sphere, Proc. Amer. Math. Soc., 10:621626.

Spanier E. (1966) - Algebraic topology, McGraw-Hill, New-York.

Steenrod N. (1951) - The topology of fibre bundles, Princeton Univ. Press.

Vaccaro M. (1957) - Propietà topologiche delle rappresentazioni localmente biunivoche, Math. Ann., 133:173-184. 
Apéndice

Seja F:M $\rightarrow \mathbb{R}$ Morse com M uma 3-variedade fechada, conexa E orientada, E, sejom $Q_{1}<\ldots<a$ valores regulares of $F$. Além disso, seja $c_{i}$ curva fechada simples em $F^{-1}\left(a_{i}\right)$. Entäo, existe $f: M \rightarrow \mathbb{R}^{2}$ genérica, $t_{A} L$ que $f_{1}=F ;(a, 0) e^{\prime}$ valor regularde $f \in c_{i}$ É UMA Componente de fle $\left.i{ }^{-1}, 0\right), \forall i$.

Prova:

Sejam $b_{1}<\ldots<b_{k}$ valores criticos de $F$ e tomemos vizinhancas $I_{j} d \in b_{j}$ en $\mathbb{R}$ com $\cap I_{j}=\phi \in U I_{j}$ ño contem $a_{i} \cdot \operatorname{Seja} M_{j}=F^{-1}\left(I_{j}\right)$.

Inicialmente constrvimos fo sobre $M_{j}$ como segue : para uma componente de $M_{j}$ que Nä́ contenha o ponto aritico de $F$ em M $M_{j}$ esta é difeomorfa 4 (superfície fechada e orientrda) x (interanto). Assim, escolhemos uma funcẽo de Morse sobre tal superaficie e définimos $t_{2}$ como sendo tal funcä́. So $M_{j}$ É una componento contendo o vitico

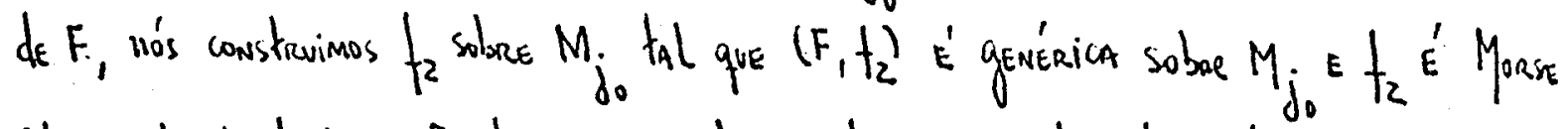
sobre o bordo de $M_{j_{0}}$. Existem 3 casos de acordo com o indice do ponto crítico EM $M_{j}$ - nós palemos construir tal $t_{2}$ de forma standard em cada caso. Agora, construimos $f_{2}$ sobre $F^{-1}\left(a_{i}\right)$. É facil ver que existe uma funcäo de Morse sobre $F^{-1}\left(a_{i}\right) \ln l$ que $c_{i}$ é componente da imajem inversa do valor regular zero. Entäo definimos to como sondo tal funcás sobre $F^{-1}\left(e_{i}\right)$. Final $E_{E}$, vamos definir $t_{2}$ sobre o conjunto intermediáRio. SEja $M_{0}$ componente dE M- $\left(U M_{j} \cup U F^{-1}\left(a_{i}\right)\right)$. Seja $N_{0}$ o fecho de $M_{0} .06$. servemos que $N_{0}$ é difeomorfo a (suporficue fech. E orient.) x (intervalo) E que sober o bardo de $N_{0} t_{2} j^{\prime}$ foi definida é Morse. Como $\mathbb{R}$ é contratil nós podemos conectar duas funióes de Morse sobre duas componentes conexas do bordo de $N_{0}$. Por um argumento de CER (1970) escolhemos uma homótopia (superficie fechada) $\times$ (intervalo) $\rightarrow \mathbb{R} \times$ (interivalo) que é genérica. Definimos entäo to por tal homutopia sobre $N_{0}$. Portanto, $t_{\text {emos }} f_{2}$

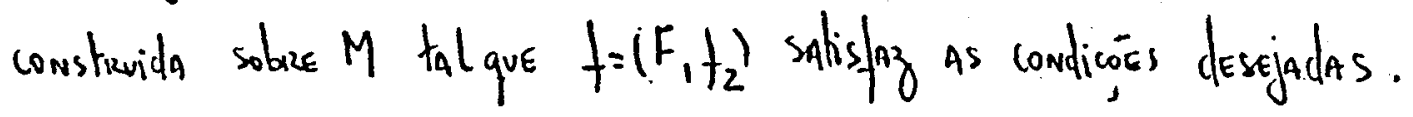

